
This book was prepared as an acccunt of work soonsored by an agency of the United States Government.
Neither the United States Government nor any agency thereot, nor any of their emplovees. makes any warranty, express or implied, or assumes any leyal liabilicy or responsibility for the accuracy.

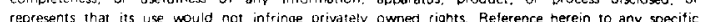
commercial prods rot necessorily consistute or imply its endorsement, recommendation, or favoring by the United States Government or any azency thereof. The views and apinions of aushors expressed herein do not

\title{
Summary of LOGDEX Data Base
}

\author{
Tom Hill and Kamy Sepehrnoori \\ Principal Investigators
Geopressured Geothermal Information System Center for Energy Studies
The University of Texas at Austin $6,4, b 3$ Austin, Texas 78712
$(512-471-4946)$

\author{
Contributors: \\ Eddie Parker \\ Jeff Potter \\ Robert Schneider
}

This work was sponsored in part by the Department of Energy contracts DE-AS-05-76ET28466 and DE-AC08-79ET27018.

Date Published - August 1981 


\section{DISCLAIMER}

This report was prepared as an account of work sponsored by an agency of the United States Government. Neither the United States Government nor any agency Thereof, nor any of their employees, makes any warranty, express or implied, or assumes any legal liability or responsibility for the accuracy, completeness, or usefulness of any information, apparatus, product, or process disclosed, or represents that its use would not infringe privately owned rights. Reference herein to any specific commercial product, process, or service by trade name, trademark, manufacturer, or otherwise does not necessarily constitute or imply its endorsement, recommendation, or favoring by the United States Government or any agency thereof. The views and opinions of authors expressed herein do not necessarily state or reflect those of the United States Government or any agency thereof. 


\section{DISCLAIMER}

Portions of this document may be illegible in electronic image products. Images are produced from the best available original document. 


\section{TABLE OF CONTENTS}

1.0 Introduction ....................... 1

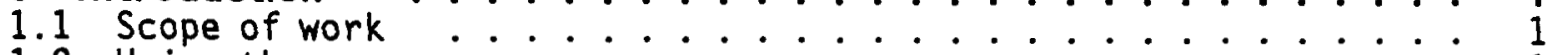

1.2 Using the summary ...................... . . 1

1.3 Data components . . . . . . . . . . . . . . . 2

1.4 Digitized $\log$ availability .................... 3

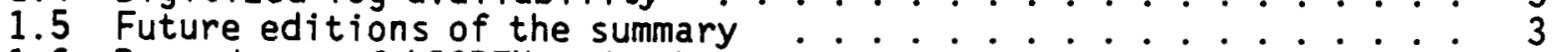

1.6 Byproducts of LOGDEX and other services . . . . . . . . . . . 3

2.0 Summary listing ................... 5

3.0 Appendix A ..................... 215

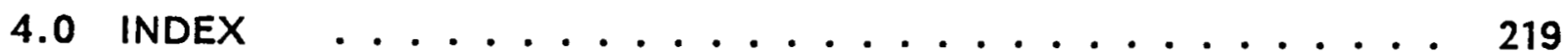




\subsection{Introduction}

This document is a summary of LOGDEX, the digitized well log data base maintained by the Center for Energy Studies at The University of Texas at Austin. These well logs were obtained from various oil companies and then converted from paper well logs to numeric information on magnetic computer tapes for input into the well log data base. This data base serves as a resource for application programs in the study of geopressured geothermal energy resources, for well logging research, and for geological research.

\subsection{Scope of work}

Currently the location and scope of well log data that may be found within the LOGDEX data base are 1 imited to wells along the Texas-Louisiana Guif Coast that are known to have a potential as a geopressured geothermal energy resource. Additionally the location of these wells in that area is highly localized into areas that have been defined by Department of Energy researchers as having a high potential for geopressured geothermal energy. The data base is being continuously expanded, and as more wells are added to the data base the distribution along the Gulf Coast should become more uniform.

The LOGDEX data base currently contains data from more than 350 wells, representing more than 1600 logs and 16,600,000 curve feet of data.

\subsection{Using the summary}

This summary is divided into three major sections: the introduction, the summary listing, and the indexes.

The introduction is intended to serve as a guide to the summary listing. This section tells the reader how the summary is organized, what may be found in it, and how the summary may be used to retrieve information about a particular log. The introduction also describes how the summary was compiled, how it might be used, and what the future plans are for it. The introduction also gives the reader any details he/she may need to know about how to use and understand what the summary contains.

The summary listing is the main body of this document. This listing was compiled from a data base that has all available information about each well log. In this listing the information has been ordered and formatted so that it may be presented in a useful way.

For quick reference to a given log, the summary listing has been indexed into seven divisions: well classification, location by county or parish, curve type, log type, operators, location by state, and well names. These indexes are arranged alphabetically and cross-referenced by page number. 
Summary of LOGDEX Data Base

\subsection{Data components}

This summary is compiled from a computer data base that contains all pertinent information about each well log. This information is then listed in the summary for each well. However, not every well listing will have every possible data component defined in the data base. As an example, it is possible that the state and county API codes may be defined for a given well but the well may not have the unique well identification code assigned by API.

The first line of each well log listing is the "LOGDEX unique identification number-." This number is important in that it is used as a reference by application programs which use the digitized well log data base. The number is unique to each well that is assigned by the Center for Energy Studies and is used as a reference only to that well. A user who knows this number is able to reference any well $\log$ within the digitized well log data base.

The next line following the LOGDEX unique number is the name of the operator or company that drilled the well. This name may be found within the listing prefixed by "Operator/Company-." In most cases this is the name of the operator or company that owned the well at the time it was drilled and does not reflect any changes in ownership of the well since that time.

Immediately below the name of the operator or company will be the well name. This name may be found within the listing prefixed by "Well name-." The well number will be suffixed to the well name in the listing if the well number is present.

The location of the well by county or parish within a state may be found within the listing prefixed by "Location-." This location will be the county and state where the well is located.

The location of the well by township-range-section within a given state may be found below the location line prefixed by "Township-range-section location-." This is the township-range-section location as reported by the operator or as located on GEOMAP well location charts.

If the abstract, tract, or block number in which the well is located is present in the data base, it may be found in the listing below the township-range-section location prefixed by "Abstract/tract/block-."

The API number of the well may be found in the listing prefixed by "API number-." The data base does not contain unique well codes as assigned by API for all wells within the data base. If this code is missing from the data base for a particular well, the space in which that code should be listed will be blank.

If the HEADER data base unique number for a particular well is present in the data base, it may be found in the listing prefixed by "HEADER data base unique number-." The HEADER data base is another data base maintained by the Center for Energy Studies which contains well log information. The major content of the HEADER data base is the $10 \mathrm{~g}$ header information, such as bottom hole temperature and the resistivity of the mud filtrate ( Rmf), for each run where information was obtained. As with the LOGDEX data base, each well in the HEADER data base has a unique number assigned to it. If the HEADER data base has information on the same well that the summary listing 
of the LOGDEX data base is displaying, the unique number of the HEADER data base will be printed so that it may be cross-referenced for further information about the well.

If the date of completion of the well is present within the data base, it may be found within the summary listing prefixed by "Date of completion-." This is the month, day, and year of completion of the well.

The well classification code of the well may be found within the listing prefixed by "Well classification code-." This classification code defines what type of well it is. For an explanation of well types please see Appendix A.

The summary will then list the $\log$ and curve types that are present in the digitized well log data base. For each log type, there may be multiple curve types. The summary will list the log type prefixed by "Log type-." Immediately below the log type will be the beginning and ending depth that the log is available for. Below this depth range will be a listing of the curve types available for this log type. These curve types will be prefixed by "Curve type(s)-" and are simply the logs that were run for this log type. This process will be repeated for each log type that is available for a given well.

\subsection{Digitized log availability}

At the present time, the digitized log data base is available only to persons with access to the computational resources at The University of Texas at Austin. For further information on the availability of this document and access to the digitized well log data base, please contact:

Geopressured Geothermal Information System

Center for Energy Studies

The University of Texas at Austin

ENS 143

Austin, Texas 78712

$(512-471-4946)$

\subsection{Future editions of the summary}

The digitized well log data base is being continually expanded. As more well logs are entered into the data base, either this summary listing will be reissued or updates will be available.

\subsection{Byproducts of LOGDEX and other services}

Application programs have been developed which make use of the digitized well $10 \mathrm{~g}$ data base. These application programs range from simply plotting the curves available for a given log to analyzing digitized well logs to generating synthetic seismograms. 
Also available are a bibliography of geopressured geothermal related topics and a thesaurus of geopressured geothermal terms. Additionally, the Center for Energy Studies maintains the Energy Information Service, which houses paper and microfiche copies of energy-related documents. 


\subsection{Summary listing}

1. LOGDEX unique identification number- 70

Operator/Company- UNION TEXAS

We 11 name- E. SUMMERS 1

Location-Brazoria County, Texas

Township-Range-Section location- 6S-38E-1

Abstract/Tract/Block-A- 100

API number- 42- 39- 5045

HEADER data base unique number- 11914

Date of completion- $1 / 9 / 67$

Well Classificattion code- GAS

Log type- Velocity

Logs available from 1576 feet to 11050 feet.

Curve type(s) available-

Self Potential

Acoustic Compensated

Caliper

Log type- Induction Electrical

Logs available from 1580 feet to 11070 feet.

Curve type(s) available-

Self Potential

Short Normal

Amplified Short Normal

Induction Conductivity

Induction Resistivity

2. LOGDEX unique identification number- 71

Operator/Company- PHILLIPS 
Summary of LOGDEX Data Base

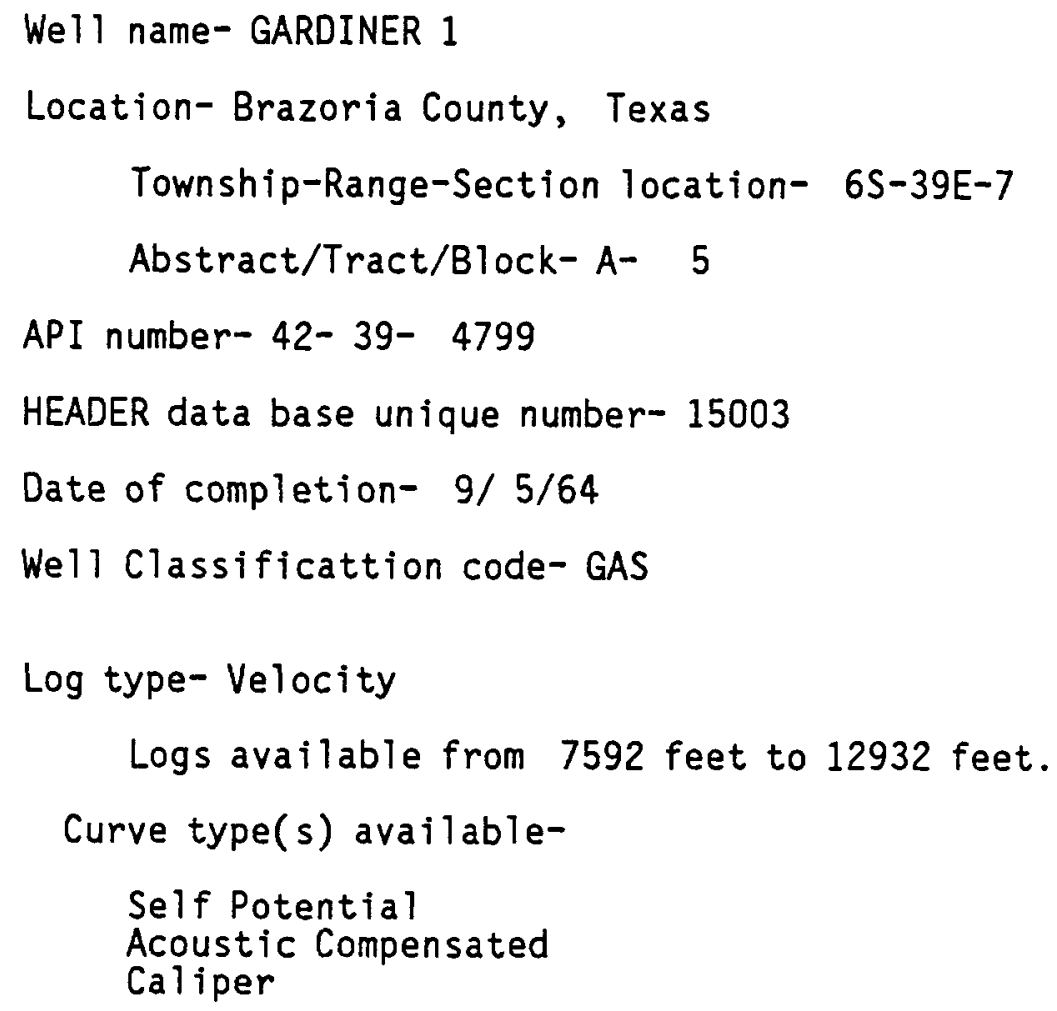

3. LOGDEX unique identification number- 72

Operator/Company- PHILLIPS.

Well name- MC ILVAINE 1

Location- Brazoria County, Texas

Township-Range-Section location- 6S-39E-6

Abstract/Tract/Block-A- 47

API number- 42- 39- 4864

HEADER data base unique number- 15259

Date of completion- 8/19/65

Well Classificattion code- D\&A

Log type-Velocity

Logs available from 7276 feet to 11894 feet. Curve type(s) available- 
Self Potential

Acoustic Compensated

Caliper

4. LOGDEX unique identification number- 73

Operator/Company- HUMBLE

We 11 name- J. SKRABANEK 1

Location-Brazoria County, Texas

Township-Range-Section location- 7S-38E-2

API number- 42- 39-

Log type- Induction Electrical

Logs available from 1950 feet to 18858 feet.

Curve type(s) available-

Self Potential

Short Normal

Amplified Short Normal

Induction Conductivity

Induction Resistivity

Log type- Velocity

Logs available from 1948 feet to 18474 feet.

Curve type(s) available-

Self Potential

Acoustic Non-Compensated

Caliper

5. LOGDEX unique identification number- 74

Operator/Company- HUMBLE

Well name- R. VIEMAN 1

Location- Brazoria County, Texas

Township-Range-Section location- 7S-38E-2 
Summary of LOGDEX Data Base

Abstract/Tract/Block-A- 12

API number- 42- 39- 4265

HEADER data base unique number- 14766

Date of completion- $12 / 31 / 63$

Well Classificattion code- GAS

Log type- Velocity

Logs available from 100 feet to 15046 feet.

Curve type(s) available-

Self Potential

Acoustic Non-Compensated

Caliper

6. LOGDEX unique identification number- 75

Operator/Company- PHILLIPS

We 11 name- HOUSTON "JJ" 1

Location-Brazoria County, Texas

Township-Range-Section location- 6S-39E-7

Abstract/Tract/Block-A- 109

API number- 42- 39- 4816

HEADER data base unique number- 14977

Date of completion- $1 / 7 / 64$

Well Classificattion code- GAS

Log type- Velocity

Logs available from 4150 feet to 17020 feet.

Curve type(s) available-

Self Potential

Acoustic Compensated

Caliper

Log type- Density

Page 8 
Logs available from 10512 feet to 17016 feet.

Curve type(s) available-

Density API Units

Caliper

Monitor

7. LOGDEX unique identification number- 76

Operator/Company-SHELL

Well name- T. H. THEISS 1

Location-Hidalgo County, Texas

Township-Range-Section location- 29S-13E-1

API number- 42-215-

Log type- Velocity

Logs available from 1514 feet to 12700 feet.

Curve type(s) available-

Self Potential

Acoustic Compensated

Caliper

Gamma Ray API Units

8. LOGDEX unique identification number- 77

Operator/Company- HUMBLE

Well name- 41

Location-Brazoria County, Texas

Township-Range-Section location- 8S-37E-2

Abstract/Tract/Block-A- 96

API number- 42- 39- 3882

HEADER data base unique number- 12227

Date of completion- $3 / 13 / 62$ 
Summary of LOGDEX Data Base

Well Classificattion code- GAS

Log type- Induction Electrical

Logs available from 2580 feet to 14000 feet.

Curve type(s) available-

Self Potential

Short Normal

Amplified Short Normal

Induction Conductivity

Induction Resistivity

Log type- Velocity

Logs available from 2570 feet to 14008 feet.

Curve type(s) available-

Self Potential

Acoustic Compensated

Caliper

9. LOGDEX unique identification number- 78

Operator/Company- CONTINENTAL

Wel1 name- WHITE-FROST 1

Location-Brazoria County, Texas

Township-Range-Section location- 8S-37E-5

API number- 42- 39-

Log type- Velocity

Logs available from 2518 feet to 11534 feet.

Curve type(s) available-

Self Potential

Acoustic Compensated

Caliper

Log type- Density

Logs available from 11538 feet to 18026 feet.

Page 10 
Curve type(s) available-

Density API Units

Gamma Ray API Units

Caliper

Monitor

Log type- Induction Electrical

Logs available from 2520 feet to 18024 feet.

Curve type(s) available-

Self Potential

Short Norma 1

Ampl if ied Short Normal

Induction Conductivity

Induction Resistivity

Gamma Ray API Units

10. LOGDEX unique identification number- 79

Operator/Company-MOBIL

Well name- HALLS BAYOU RANCH 1

Location-Galveston County, Texas

Township-Range-Section location- $6 S-40 E-8$

API number- 42-167-

Log type- Induction Electrical

Logs available from 140 feet to 19014 feet.

Curve type(s) available-

Self Potential

Short Normal

Amplified Short Normal

Induction Conductivity

Induction Resistivity

Log type- Velocity

Logs available from 150 feet to 18954 feet.

Curve type(s) available- 
Summary of LOGDEX Data Base

\author{
Self Potential \\ Acoustic Compensated \\ Caliper
}

11. LOGDEX unique identification number- 80

Operator/Company- HUMBLE

We 11 name- A. B. WILLIAMSON 1

Location-Brazoria County, Texas

Township-Range-Section location- 8S-37E-3

Abstract/Tract/Block-A- 96

API number- $42-39-4470$

HEADER data base unique number- 12233

Date of completion- $7 / 8 / 60$

We11 Classificattion code- GAS

Log type- Velocity

Logs available from 2506 feet to 12508 feet.

Curve type(s) available-

Self Potential

Acoustic Compensated

Log type- Induction Electrical

Logs available from 2500 feet to 13040 feet.

Curve type(s) available-

Self Potential

Short Normal

Amplified Short Normal

Induction Conductivity

Induction Resistivity

12. LOGDEX unique identification number- 81

Operator/Company- PHILLIPS

Page 12 


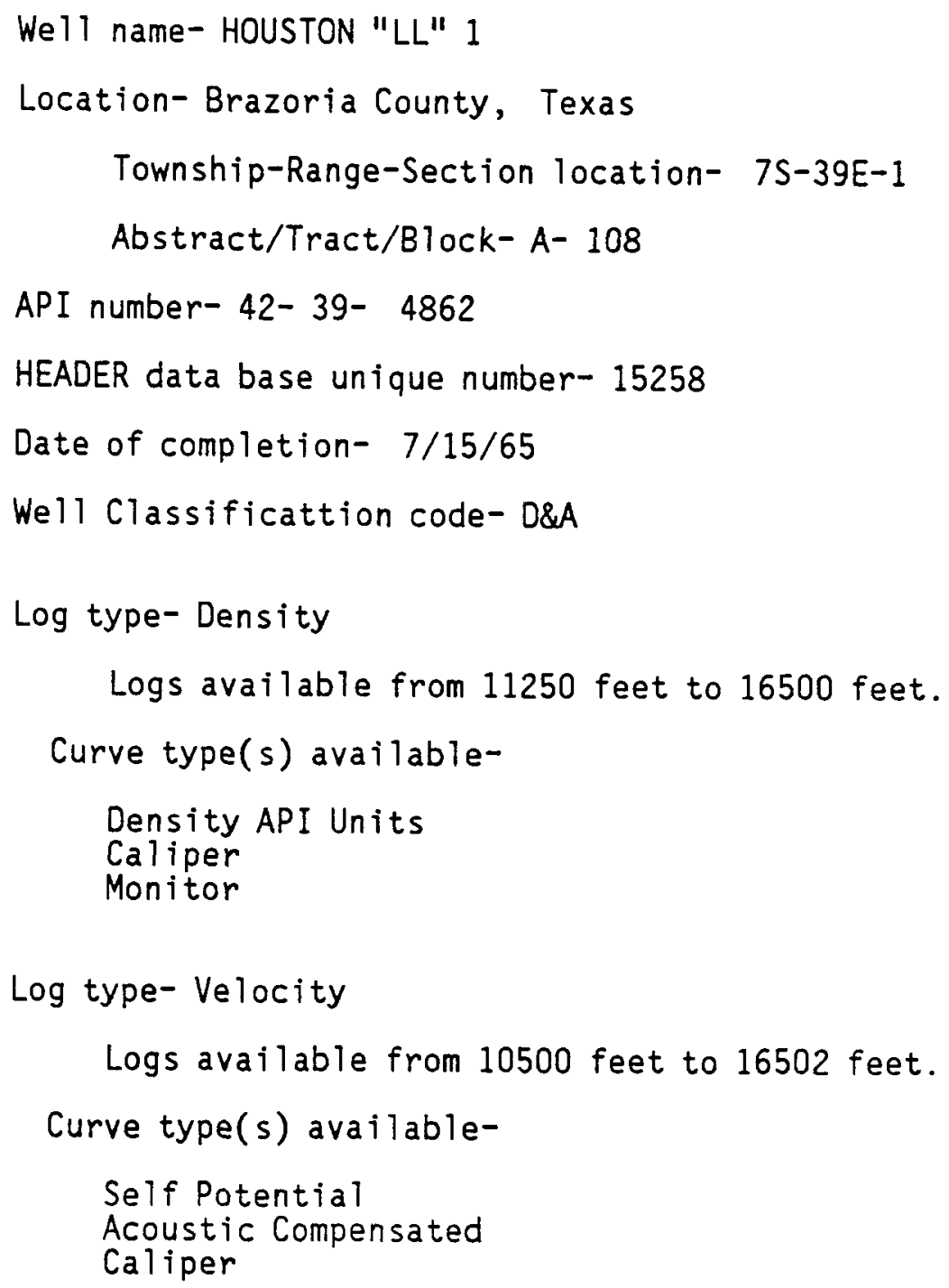

13. LOGDEX unique identification number- 82 Operator/Company- SOCONY MOBIL .

Well name- H. MC NEIL 1

Location- Brazoria County, Texas

Township-Range-Section location- 8S-37E-9

Abstract/Tract/Block-A- 120

API number- 42- 39- 132

HEADER data base unique number- 11386 
Summary of LOGDEX Data Base

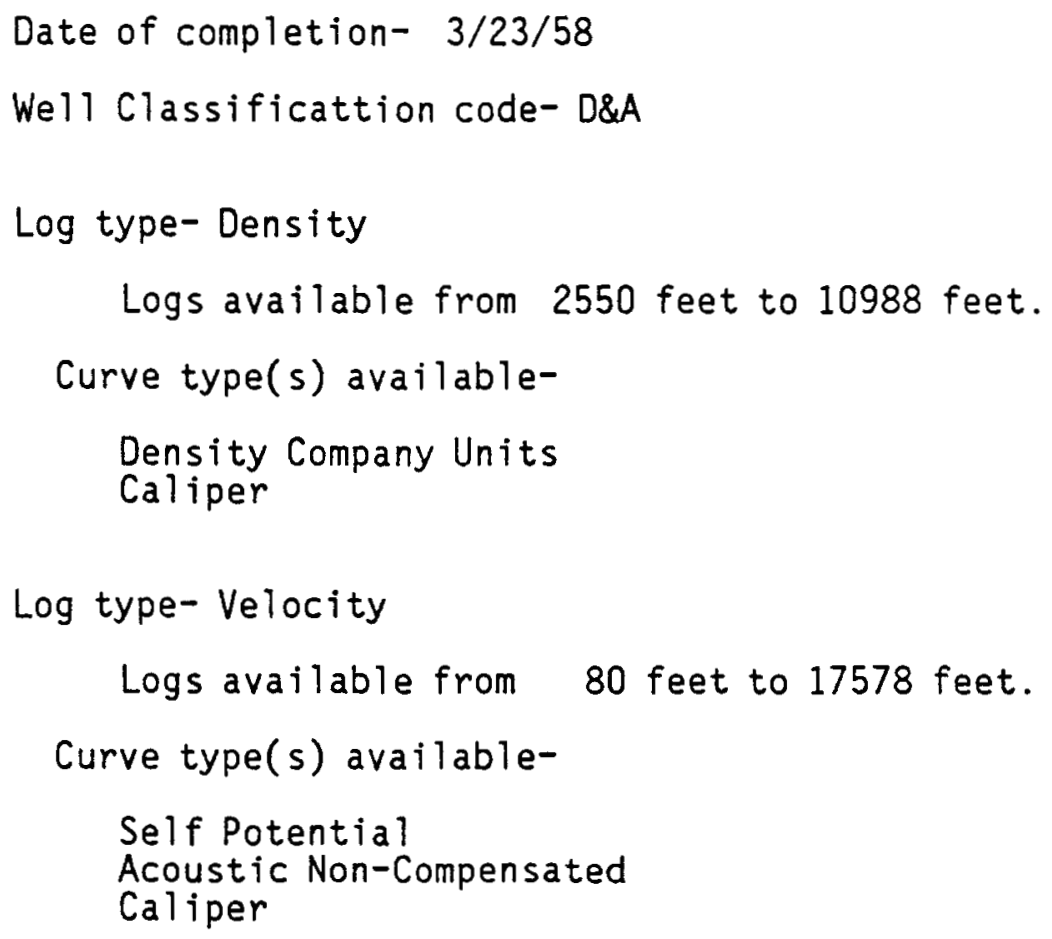

14. LOGDEX unique identification number- 83

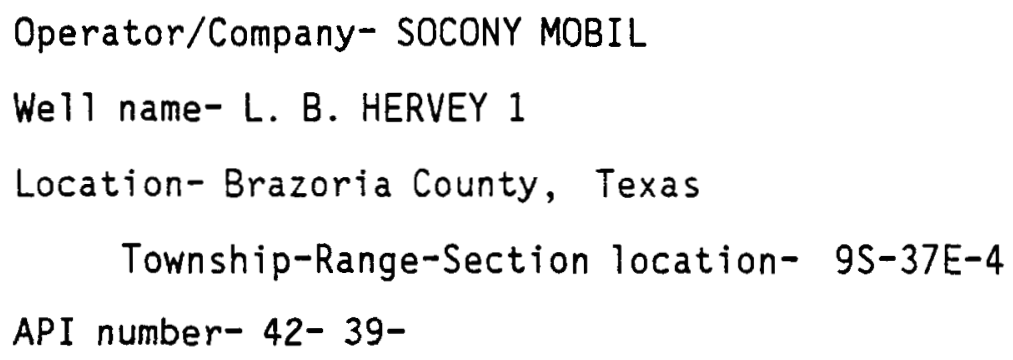


Curve type(s) available-

Self Potential

Short Normal

Amplified Short Normal

Induction Conductivity

Induction Resistivity

15. LOGDEX unique identification number- 84

Operator/Company-MOBIL

Well name- J. R. SMITH 1

Location-Brazoria County, Texas

Township-Range-Section location- 8S-36E-1

Abstract/Tract/Block-A- 19

API number- 42- 39- 30122

HEADER data base unique number- 12223

Date of completion- $8 / 18 / 70$

Well Classificattion code- GAS

Log type- Induction Electrical

Logs available from 350 feet to 13616 feet.

Curve type(s) available-

Self Potential

Short Normal

Amplified Short Normal

Induction Conductivity

Induction Resistivity

Log type-Velocity

Logs available from 3450 feet to 13606 feet.

Curve type(s) available-

Self Potential

Acoustic Compensated

Caliper 
16. LOGDEX unique identification number- 85

Operator/Company- MOBIL

We 11 name- RETRIEVE STATE FARM 1

Location-Brazoria County, Texas

Township-Range-Section location- 8S-36E-1

Abstract/Tract/Block-A- 19

API number- 42- 39- 30157

HEADER data base unique number- 12224

Date of completion- $3 / 12 / 71$

Well Classificattion code- T\&A

Log type- Velocity

Logs available from 5028 feet to 13470 feet.

Curve type(s) available-

Self Potential

Acoustic Compensated

Caliper

Log type- Induction Electrical

Logs available from 50 feet to 13468 feet.

Curve type(s) available-

Self Potential

Short Normal

Amplified Short Normal

Induction Resistivity

17. LOGDEX unique identification number- 86

Operator/Company- MOBIL

Well name- L. B. HERVEY 2

Location-Brazoria County, Texas 
Township-Range-Section location- 9S-37E-5

Abstract/Tract/Block-A- 20

API number- 42- 39- 4930

HEADER data base unique number- 12449

Date of completion- 5/30/66

Well Classificattion code- D\&A

Log type- Induction Electrical

Logs available from 3020 feet to 12512 feet.

Curve type(s) available-

Self Potential

Short Normal

Amplified Short Normal

Induction Conductivity

Induction Resistivity

Log type-Velocity

Logs available from 3030 feet to 12502 feet.

Curve type(s) available-

Self Potential

Acoustic Non-Compensated

Acoustic Compensated

Caliper

18. LOGDEX unique identification number-

87

Operator/Company- SOCONY MOBIL

Well name- J. H. DINGLE 1

Location-Brazoria County, Texas

Township-Range-Section location- 9S-37E-3

API number- 42- 39-

Log type-Velocity

Logs available from 3214 feet to 12476 feet. 
Curve type(s) available-

Self Potential

Acoustic Non-Compensated

Caliper

Log type- Induction Electrical

Logs available from 100 feet to 12480 feet.

Curve type(s) available-

Self Potential

Short Norma?

Amplified Short Normal

Induction Conductivity

Induction Resistivity

19. LOGDEX unique identification number- 88

Operator/Company- SOCONY MOBIL

Wel1 name- RETRIEVE STATE FARM 2

Location- Brazoria County, Texas

Township-Range-Section location- 8S-37E-3

Abstract/Tract/Block-A- 19

API number- 42- 39- 4473

HEADER data base unique number- 12235

Date of completion- 5/30/63

Well Classificattion code- OIL

Log type- Velocity

Logs available from 4700 feet to 13496 feet.

Curve type(s) available-

Self Potential

Acoustic Compensated

Caliper

Log type- Induction Electrical

Logs available from 160 feet to 13494 feet. 
Curve type(s) available-

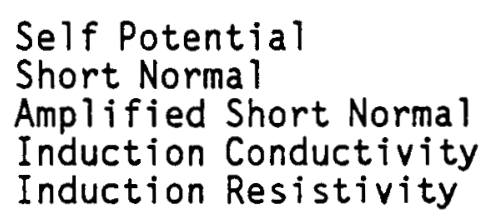

20. LOGDEX unique identification number- 90

Operator/Company- HUMBLE

We 11 name- PERRYVILLE 1

Location-Wood County, Texas

Township-Range-Section location- 23N-39E-1

API number- 42-499-

Log type- Velocity

Logs available from 2700 feet to 14132 feet.

Curve type(s) available-

Self Potential

Acoustic Compensated

Caliper

21. LOGDEX unique identification number-

91

Operator/Company- DOW

Well name- C. C. BELL 1

Location-Brazoria County, Texas

Township-Range-Section location- 9S-36E-2

API number- 42- 39-

Log type- Induction Electrical

Logs available from 4000 feet to 15562 feet. 


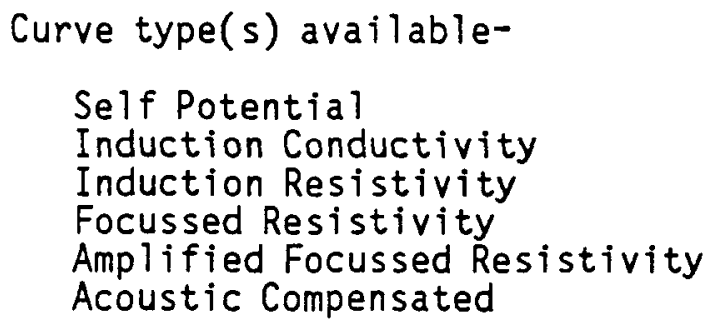

Log type- Density

Logs available from 12200 feet to 15546 feet.

Curve type(s) available-

Density API Units

Gamma Ray API Units

Caliper

Monitor

22. LOGDEX unique identification number- 92

Operator/Company- HASSIE HUNT TRUST

Well name- S. H. GREEN 3

Location- Galveston County, Texas

Township-Range-Section location- 6S-40E-2

Abstract/Tract/Block-A- 48

API number- 42-167- 30165

HEADER data base unique number- 11963

Date of completion- 12/22/74

Well Classificattion code- D\&A

Log type- Velocity

Logs available from 9078 feet to 12718 feet.

Curve type(s) available-

Acoustic Compensated

Caliper

Gamma Ray API Units

Log type- Induction Electrical 
Logs available from 1100 feet to 12732 feet.

Curve type(s) available-

Self Potential

Induction Conductivity

Induction Resistivity

Focussed Resistivity

Amplified Focussed Resistivity

Gamma Ray API Units

23. LOGDEX unique identification number- 93

Operator/Company- GULF

We 11 name- TEXAS STATE LEASE 530342

Location-Brazoria County, Texas

Township-Range-Section location- 8S-39E-2

Abstract/Tract/Block-T- 8

API number- $42-39-4515$

HEADER data base unique number- 12240

Date of completion- 5/23/63

Well Classificattion code- D\&A

Log type- Velocity

Logs available from 1052 feet to 13858 feet.

Curve type(s) available-

Self Potential

Acoustic Non-Compensated

Caliper

Log type- Induction Electrical

Logs available from 1052 feet to 14698 feet.

Curve type(s) available-

Self Potential

Short Normal

Ampl ified Short Normal

Induction Conductivity

Induction Resistivity 
24. LOGDEX unique identification number- 94

Operator/Company- HUMBLE

Well name- M. E. HUNTER 1

Location-Brazoria County, Texas

Township-Range-Section location- 7S-38E-2

Abstract/Tract/Block-A- 12

API number- 42- 39- 4805

HEADER data base unique number- 14632

Date of completion- $6 / 28 / 64$

We11 Classificattion code- D\&A

Log type- Velocity

Logs available from 5412 feet to 15456 feet.

Curve type(s) available-

Self Potential

Acoustic Non-Compensated

Caliper

Log type- Induction Electrical

Logs available from 170 feet to 16030 feet.

Curve type(s) available-

Self Potential

Short Normal

Amplified Short Normal

Induction Conductivity

Induction Resistivity

25. LOGDEX unique identification number-

Operator/Company- HUMBLE

We 11 name- WARD-BYERS 1

Location-Brazoria County, Texas 
Township-Range-Section location- 8S-36E-2

Abstract/Tract/Block-A- 19

API number- 42- 39- 3888

HEADER data base unique number- 12220

Date of completion- $1 / 19 / 58$

Well Classificattion code- D\&A

Log type-Velocity

Logs available from 2526 feet to 14536 feet.

Curve type(s) available-

Self Potential

Acoustic Non-Compensated

26. LOGDEX unique identification number- 96

Operator/Company- PHILLIPS

We11 name- TACQUARD "A" 1

Location-Galveston County, Texas

Township-Range-Section location- 6S-40E-5

Abstract/Tract/Block-A- 47

API number- 42-167- 1954

HEADER data base unique number- 11965

Date of completion- 9/29/64

Well Classificattion code- GAS

Log type- Velocity

Logs available from 10980 feet to 14506 feet.

Curve type(s) available-

Self Potential

Acoustic Non-Compensated

Caliper 
Summary of LOGEEX Data Base

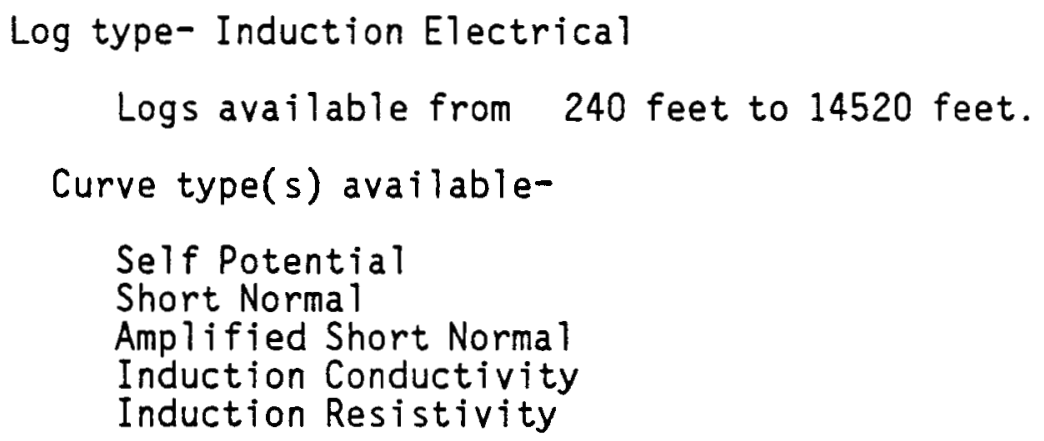

27. LOGDEX unique identification number-

Operator/Company- TEXAS EASTERN

Well name- NANA D. NEWTON 1

Location-Galveston County, Texas

Township-Range-Section location- 6S-40E-3

Abstract/Tract/Block-A- 5

API number- 42-167- 1460

HEADER data base unique number- 11930

Date of completion-12/18/61

Well Classificattion code- GAS

Log type- Velocity

Logs available from 9150 feet to 11250 feet.

Curve type(s) available-

Self Potential

Acoustic Non-Compensated .

Caliper

Log type- Induction Electrical

Logs available from 1376 feet to 11282 feet.

Curve type(s) available-

Self Potential

Short Normal

Ampl ified Short Normal

Long Normal 


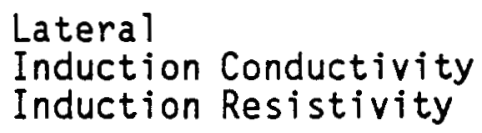

28. LOGDEX unique identification number- 98

Operator/Company- GENERAL CRUDE

Wel 1 name- T. MARTIN 1

Location-Brazoria County, Texas

Township-Range-Section location- 7S-39E-1

API number- 42- 39-

Log type- Induction Electrical

Logs available from 312 feet to 16418 feet.

Curve type(s) available-

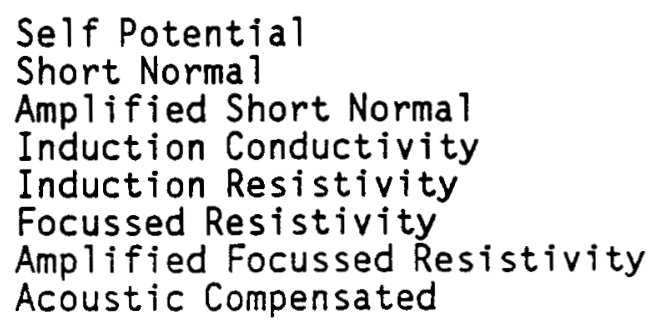

29. LOGDEX unique identification number-

Operator/Company- TENNECO

Well name- AMERICAN FLECTCHER 1

Location-Brazoria County, Texas

Township-Range-Section location- 8S-38E-7

Abstract/Tract/B1ock-A- 261

API number- 42- 39- 58

HEADER data base unique number- 11387

Date of completion- $10 / 19 / 53$ 


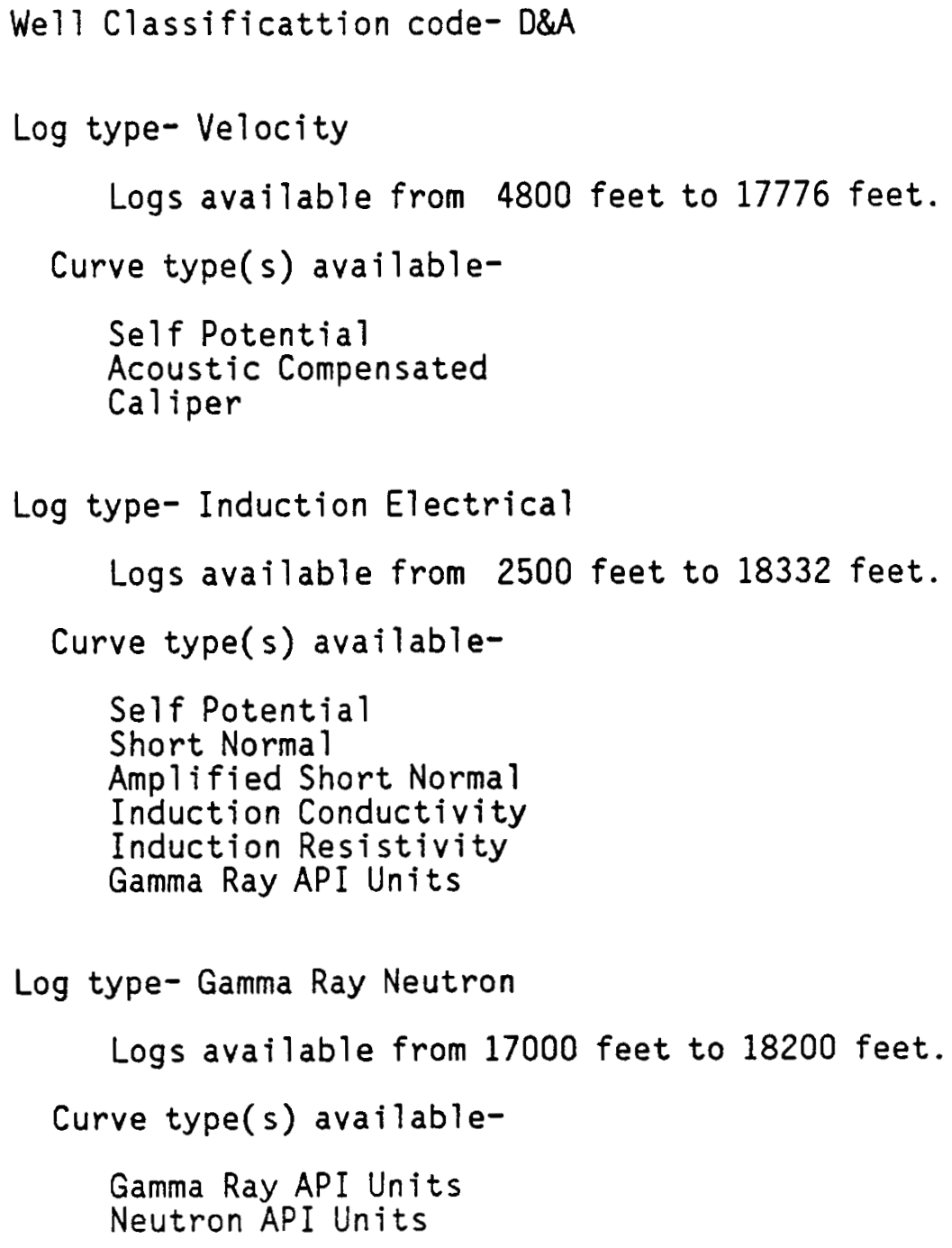

Log type- Gamma Ray Neutron

Logs available from 17000 feet to 18200 feet.

Curve type(s) available-

Gamma Ray API Units

Neutron API Units

30. LOGDEX unique identification number- 100

Operator/Company- PAN AMERICAN

We 11 name- IDA HOBBS 1

Location-Brazoria County, Texas

Township-Range-Section location- 8S-36E-4

Abstract/Tract/B1ock-A- 20

API number- $42-39-3898$

HEADER data base unique number- 12221

Date of completion- $4 / 19 / 63$ 
We1l Classificattion code- J\&A

Log type- Velocity

Logs available from 3540 feet to 12282 feet.

Curve type(s) available-

Self Potential

Acoustic Non-Compensated

Caliper

Log type- Induction Electrical

Logs available from 120 feet to 12272 feet.

Curve type(s) available-

Self Potential

Short Norma 1

Amplified Short Normal

Induction Conductivity

Induction Resistivity

Log type- Micro

Logs available from 9400 feet to 11534 feet.

Curve type(s) available-

Self Potential

Inverse 1 Inch

Caliper

31. LOGDEX unique identification number- 101

Operator/Company- DAVIS

Well name- GALAZNIK 1

Location-Brazoria County, Texas

Township-Range-Section location- 7S-37E-5

Abstract/Tract/Block-A- 380

API number- 42- 39- 30432

HEADER data base unique number- 12055

Date of completion- $2 / 6 / 74$ 
Summary of LOGDEX Data Base

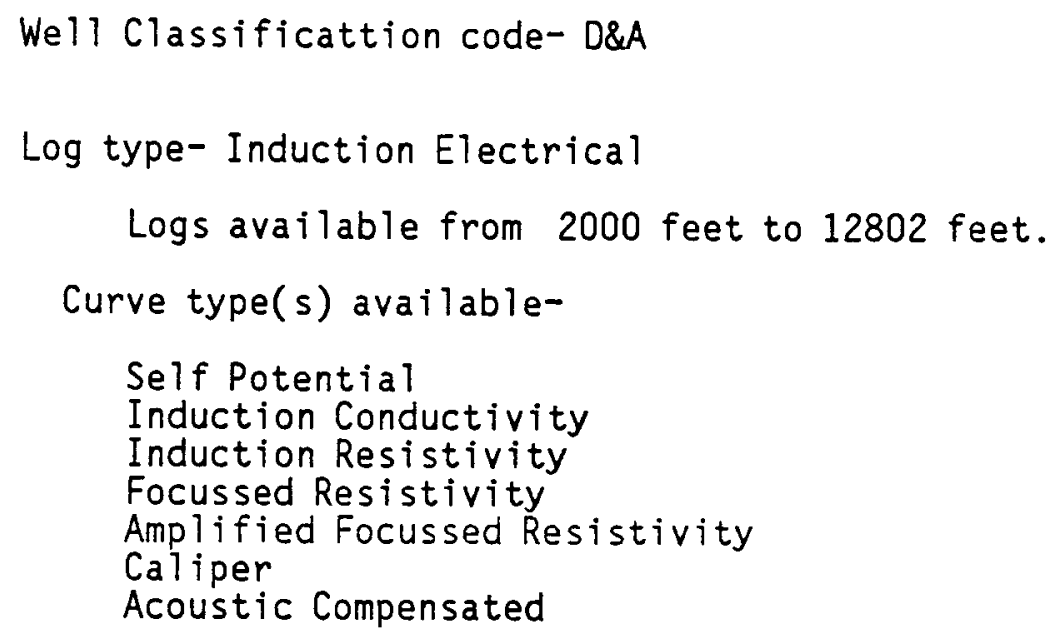

32. LOGDEX unique identification number- 102

Operator/Company- HUMBLE

Well name- LEE 1

Location-Brazoria County, Texas

Township-Range-Section location- 7S-37E-5

Abstract/Tract/Block-A- 318

API number- 42- 39- 4203

HEADER data base unique number- 12053

Date of completion- $5 / 4 / 62$

Well Classificattion code- D\&A

Log type- Velocity

Logs available from 3244 feet to 13106 feet.

Curve type(s) available-

\section{Self Potential}

Acoustic Non-Compensated

Caliper

Log type- Induction Electrical

Logs available from 3246 feet to 13110 feet.

Curve type(s) available- 
Self Potential

Short Norma 1

Amplified Short Normal

Induction Conductivity

Induction Resistivity

33. LOGDEX unique identification number- 103

Operator/Company - HOUSTON OIL AND MINERALS

Wel1 name- FREEPORT SULFUR 1

Location-Brazoria County, Texas

Township-Range-Section location- 9S-37E-6

Abstract/Tract/Block-A- 142

API number- 42- 39- 30468

HEADER data base unique number- 11426

Date of completion- $7 / 3 / 74$

Well Classificattion code- D\&A

Log type- Induction Electrical

Logs available from 200 feet to 16932 feet.

Curve type(s) available-

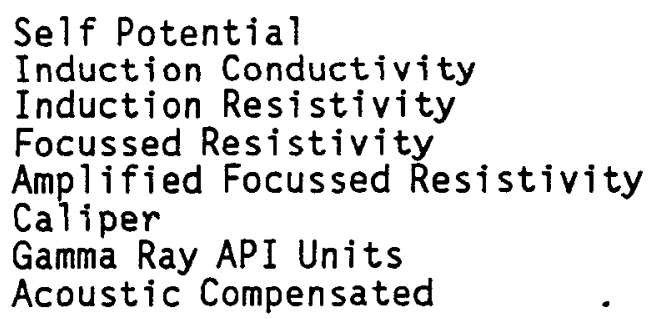

34. LOGDEX unique identification number- 104

Operator/Company- HUMBLE

Well name- FREEPORT SULPHUR 1

Location-Brazoria County, Texas 
Summary of LOGDEX Data Base

Township-Range-Section location- 9S-37E-6

API number- 42- 39-

Log type- Induction Electrical

Logs available from 3020 feet to 14060 feet.

Curve type(s) available-

Self Potential

Short Norma 1

Amplified Short Normal

Induction Conductivity

Induction Resistivity

Gamma Ray API Units

Log type- Velocity

Logs available from 3012 feet to 14050 feet.

Curve type(s) available-

Self Potential

Acoustic Non-Compensated

Caliper

Gamma Ray API Units

35. LOGDEX unique identification number- 105

Operator/Company- PHILLIPS

Well name- HOUSTON "M" 2

Location- Brazoria County, Texas

Township-Range-Section location- 6S-39E-4

Abstract/Tract/Block- A- $110^{\circ}$

API number- 42- 39- 984

HEADER data base unique number- 14978

Date of completion- 9/30/56

Well Classificattion code- GAS

Log type- Micro 
Logs available from 10200 feet to 17264 feet.

Curve type(s) available-

Self Potential

Inverse 1 Inch

Caliper

Log type- Electrical

Logs available from 200 feet to 18006 feet.

Curve type(s) available-

Self Potential

Short Norma 1

Amp lified Short Normal

Long Norma 1

Lateral

Log type- Gamma Ray Neutron

Logs avajlable from 8900 feet to 11730 feet.

Curve type(s) available-

Gamma Ray Company Units

Neutron Company Units

36. LOGDEX unique identification number- 106

Operator/Company- PHILLIPS

We11 name- PABST "B" 2

Location-Galveston County, Texas

Township-Range-Section location- 6S-40E-3

API number- 42-167-

Log type- Induction Electrical

Logs available from 228 feet to 12122 feet.

Curve type(s) available-

Self Potential

Short Normal

Amplified Short Normal 


\section{Induction Conductivity Induction Resistivity}

Log type- Velocity

Logs available from 6846 feet to 12720 feet.

Curve type(s) available-

Self Potential

Acoustic Non-Compensated

Caliper

37. LOGDEX unique identification number- 107

Operator/Company- GENERAL CRUDE

We 11 name- T. MARTIN 3

Location-Brazoria County, Texas

Township-Range-Section location- 7S-39E-6

API number- 42- 39-

Log type- Induction Electrical

Logs available from 348 feet to 16034 feet.

Curve type(s) available-

Self Potential

Induction Conductivity

Induction Resistivity

Focussed Resistivity

Amplified Focussed Resistivity

Acoustic Compensated

38. LOGDEX unique identification number- 108

Operator/Company- GENERAL CRUDE

We 11 name- T. MARTIN 2

Location-Brazoria County, Texas

Township-Range-Section location- 7S-39E-1 
API number- 42- 39-

Log type- Induction Electrical

Logs available from 322 feet to 17150 feet.

Curve type(s) available-

Self Potential

Short Normal

Amplified Short Normal

Induction Conductivity

Induction Resistivity

Focussed Resistivity

Amplified Focussed Resistivity

Acoustic Compensated

39. LOGDEX unique identification number- 109

Operator/Company- TEXAS EASTERN

We 11 name- HITCHCOCK 1

Location-Galveston County, Texas

Township-Range-Section location- 6S-40E-1

Abstract/Tract/Block- A- 193

API number- 42-167- 1383

HEADER data base unique number- 11953

Date of completion- 7/27/63

Well Classificattion code- D\&A

Log type- Induction Electrical

Logs available from 260 feet to 14512 feet.

Curve type(s) available-

Self Potential

Short Normal

Ampl if ied Short Normal

Induction Conductivity

Induction Resistivity

Log type-Velocity 
Summary of LOGDEX Data Base

Logs available from 10234 feet to 14502 feet.

Curve type(s) available-

Self Potential

Acoustic Non-Compensated

Caliper

40. LOGDEX unique identification number- 110

Operator/Company-MCCULLOCH

Well name- LOBIT 1

Location-Galveston County, Texas

Township-Range-Section location- 7S-40E-1

API number- 42-167-

Log type- Induction Electrical

Logs available from 1490 feet to 18468 feet.

Curve type(s) available-

Self Potential

Induction Conductivity

Induction Resistivity

Focussed Resistivity

Amplified Focussed Resistivity

Gamma Ray API Units

Log type- Density

Logs available from 14964 feet to 18142 feet.

Curve type(s) available-

Density API Units

Monitor

41. LOGDEX unique identification number- 111

Operator/Company- HUMBLE

Well name- STATE TRACT 811

Page 34 


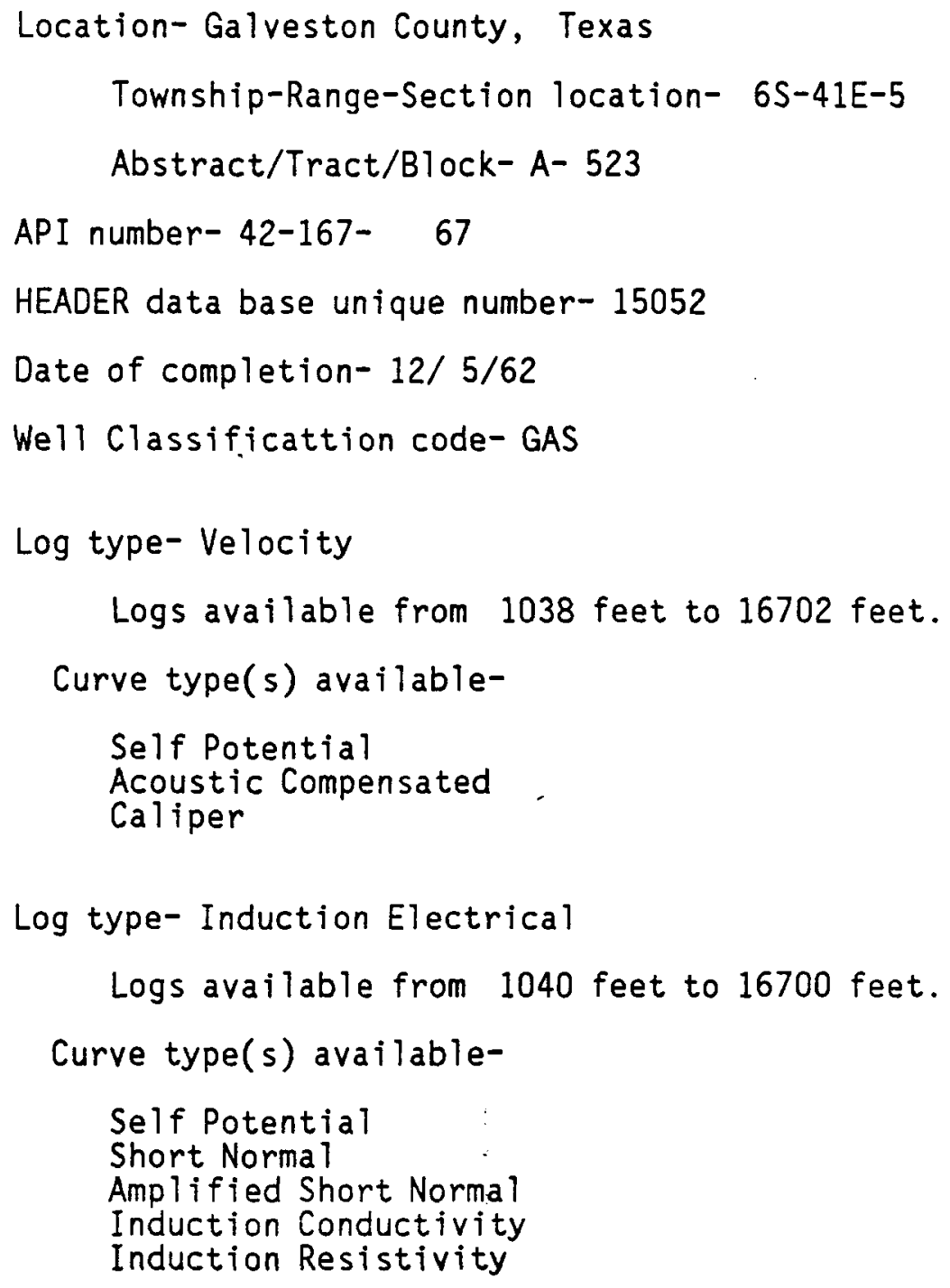

Log type- Induction Electrical

Logs available from 1040 feet to 16700 feet.

Curve type(s) available-

Self Potential

Short Normal

Amplified Short Normal

Induction Conductivity

Induction Resistivity

42. LOGDEX unique identification number- 112 Operator/Company- BURNS TRUST \#2 .

We 11 name- POTTER 1

Location-Brazoria County, Texas

Township-Range-Section location- 6S-39E-3

Abstract/Tract/Block- A- 456

API number- 42- 39- 5004

HEADER data base unique number- 14983 
Summary of LOGDEX Data Base

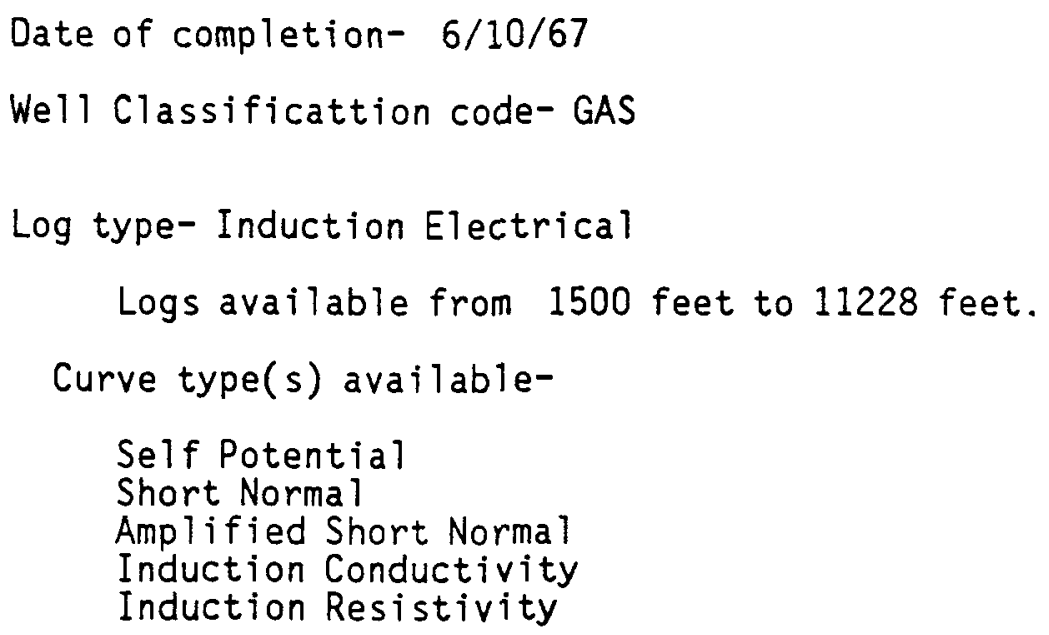

43. LOGDEX unique identification number- 113

Operator/Company- AMBASSADOR OIL CORPORATION

We 11 name- FREEMAN E. PERKINS ET AL 1

Location-Brazoria County, Texas

Township-Range-Section location- 6S-39E-2

Abstract/Tract/Block-A- 431

API number- 42- 39- 4822

HEADER data base unique number- 14975

Date of completion-12/14/64

Well Classificattion code- D\&A

Log type- Induction Electrical

Logs available from 1400 feet to 9020 feet.

Curve type(s) available-

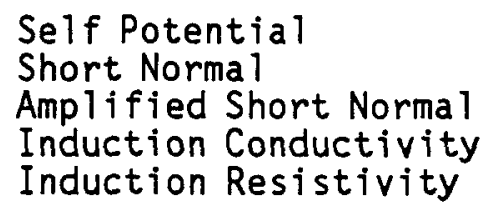


44. LOGDEX unique identification number- 114

Operator/Company- QUINTANA PETROLEUM CORPORATION

We 11 name- CLYDE HERRING 1

Location-Brazoria County, Texas

Township-Range-Section location- 6S-39E-3

Abstract/Tract/Block-A- 497

API number- 42- 39- 4744

HEADER data base unique number- 14980

Date of completion- $1 / 17 / 64$

Well Classificattion code- D\&A

Log type- Induction Electrical

Logs avajlable from 450 feet to 12088 feet.

Curve type(s) available-

Self Potential
Short Normal
Amplified Short Normal
Induction Conductivity
Induction Resistivity

45. LOGDEX unique identification number- 115

Operator/Company- BURNS TRUST NO. TWO

Wel 1 name- TRIANGLE 1

Location-Brazoria County, Texas

Township-Range-Section location- 6S-39E-3

Abstract/Tract/Block-A- 456

API number- 42- 39- 5103

HEADER data base unique number- 14920

Date of completion- 11/29/68

Well Classificattion code- D\&A 
Log type- Induction Electrical

Logs available from 1540 feet to 10238 feet.

Curve type(s) available-

Self Potential

Short Normal

Ampl ified Short Normal

Induction Conductivity

Induction Resistivity

46. LOGDEX unique identification number- 116

Operator/Company- NORTH CENTRAL OIL CORPORATION

Well name- L. B. HUBBARD 1

Location- Brazoria County, Texas

Township-Range-Section location- 6S-38E-1

Abstract/Tract/Block-A- 72

API number- $42-39-4759$

HEADER data base unique number- 11909

Date of completion- $4 / 14 / 64$

Well Classificattion code- D\&A

Log type- Induction Electrical

Logs available from 1310 feet to 10220 feet.

Curve type(s) available-

Self Potential

Short Norma 1

Amplified Short Normal

Induction Conductivity

Induction Resistivity

47. LOGDEX unique identification number- 117

Operator/Company - TEXKAN OIL COMPANY

Well name- MARIE KINAT LORENZ 1

Page 38 
Location-Brazoria County, Texas

Township-Range-Section location- 6S-38E-1

Abstract/Tract/Block-A- 429

API number- 42- 39- 1536

HEADER data base unique number- 11920

Date of completion-11/4/61

Well Classificattion code- J\&A

Log type- Induction Electrical

Logs available from 1498 feet to 10732 feet.

Curve type(s) available-

Self Potential

Short Normal

Amplified Short Normal

Induction Conductivity

Induction Resistivity

48. LOGDEX unique identification number- 118

Operator/Company- BUTTES

We 11 name-A. B. MARSHALL 1

Location-Brazoria County, Texas

Township-Range-Section location- 6S-40E-4

API number- 42- 39-

Log type- Induction Electrical •

Logs available from 300 feet to 14364 feet.

Curve type(s) available-

Self Potential

Short Normal

Amplified Short Normal

Induction Conductivity

Induction Resistivity 
Summary of LOGDEX Data Base

49. LOGDEX unique identification number- 119

Operator/Company-W. CROSBY

Well name-WILSON 1

Location- Brazoria County, Texas

Township-Range-Section location- 6S-39E-5

API number- 42- 39-

Log type- Electrical

Logs available from 2914 feet to 10474 feet.

Curve type(s) available-

Self Potential

Short Norma 1

Long Normal

Lateral

50. LOGDEX unique identification number- 120

Operator/Company- PHILLIPS

Well name- ROBNETT 1

Location-Brazoria County, Texas

Township-Range-Section location- 6S-39E-2

API number- 42- 39-

Log type- Electrical

Logs available from 1454 feet to 12590 feet.

Curve type(s) available-

Self Potential

Short Normal

Amplified Short Normal

Long Norma 1

Lateral

Page 40 
51. LOGDEX unique identification number- 121

Operator/Company- BUTTES

We 11 name- A. B. MARSHALL 3

Location-Galveston County, Texas

Township-Range-Section location- 6S-40E-9

API number- 42-167-

Log type- Induction Electrical

Logs available from 300 feet to 15584 feet.

Curve type(s) available-

Self Potential

Short Norma 1

Ampl if ied Short Normal

Induction Conductivity

Induction Resistivity

52. LOGDEX unique identification number- 122

Operator/Company- TEXACO

We 11 name- J. W. HARRIS ET AL 1

Location-Brazoria County, Texas

Township-Range-Section location- 6S-39E-6

Abstract/Tract/Block-A- 47

API number- 42- 39- 1024

HEADER data base unique number- 14974

Date of completion- $3 / 4 / 52$

Well Classificattion code- OIL

Log type- Electrical

Logs available from 2042 feet to 11968 feet. 
Summary of LOGDEX Data Base

Curve type(s) available-

Self Potential

Short Normal

Ampl ified Short Normal

Long Normal

Lateral

53. LOGDEX unique identification number- 123

Operator/Company- PHILLIPS

We 11 name- KENTZELMAN 1

Location-Brazoria County, Texas

Township-Range-Section location- 6S-39E-2

API number- 42- 39-

Log type- Electrical

Logs available from 1430 feet to 11608 feet.

Curve type(s) available-

Self Potential

Short Normal

Ampl ified Short Normal

Long Norma 1

Lateral

54. LOGDEX unique identification number- 124

Operator/Company- HUMBLE

We 11 name- RETRIEVE STATE FARM TRACT \#5. 1

Location- Brazoria County, Texas

Township-Range-Section location- 8S-36E-6

Abstract/Tract/Block- A- 343

API number- 42- 39- 51

HEADER data base unique number- 11385

Page 42 


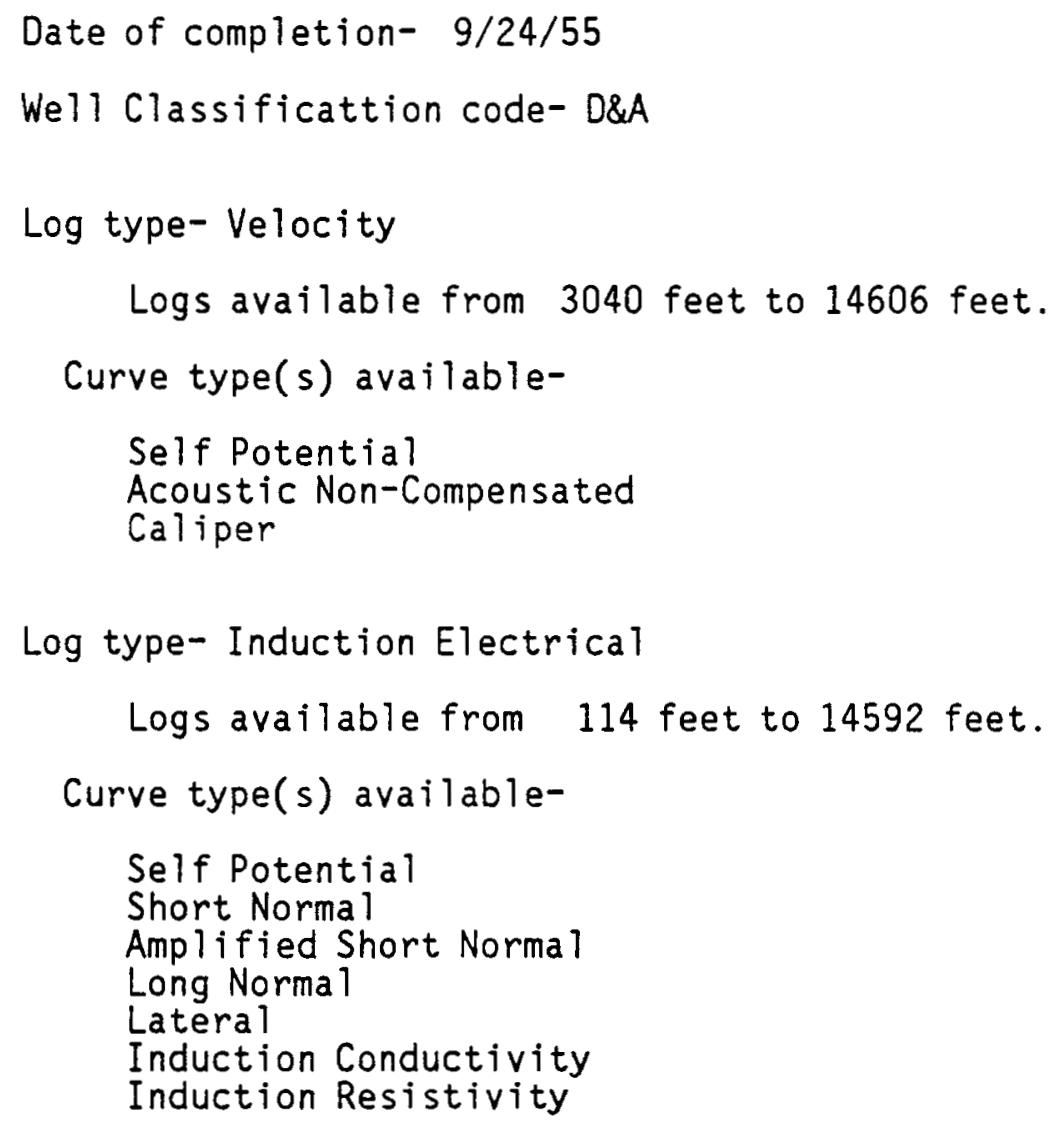

55. LOGDEX unique identification number- 126

Operator/Company- PAN AMERICAN

We 11 name- B. R. L. D. CO. A-1

Location-Brazoria County, Texas

Township-Range-Section location- 8S-35E-8

Abstract/Tract/Block-A- 78.

API number- 42- 39- 4069

HEADER data base unique number- 12212

Date of completion- $3 / 1 / 57$

Well Classificattion code- GAS

Log type- Induction Electrical

Logs available from 120 feet to 14010 feet. 


$$
\begin{aligned}
& \text { Curve type(s) available- } \\
& \text { Self Potential } \\
& \text { Short Normal } \\
& \text { Amplified Short Normal } \\
& \text { Long Normal } \\
& \text { Lateral } \\
& \text { Induction Conductivity } \\
& \text { Induction Resistivity }
\end{aligned}
$$

56. LOGDEX unique identification number- 127

Operator/Company-MICHAEL T. HALBOUTY

We11 name-MARIE D. ELLIS 1

Location-Brazoria County, Texas

Township-Range-Section location- 8S-35E-5

Abstract/Tract/Block-A- 85

API number- 42- 39- 30402

HEADER data base unique number- 12214

Date of completion- 2/28/73

Well Classificattion code- D\&A

Log type- Induction Electrical

Logs available from 2070 feet to 12104 feet.

Curve type(s) available-

$$
\begin{aligned}
& \text { Self Potential } \\
& \text { Induction Conductivity } \\
& \text { Induction Resistivity } \\
& \text { Focussed Resistivity }
\end{aligned}
$$

57. LOGDEX unique identification number- 128

Operator/Company- PAN AMERICAN

Wel1 name- I. A. MELLON, ET AL 1

Location-Brazoria County, Texas 
Township-Range-Section location- 9S-35E-1

API number- 42- 39-

Log type- Induction Electrical

Logs available from 2528 feet to 16040 feet.

Curve type(s) available-

Self Potential

Short Normal

Amplified Short Normal

Induction Conductivity

Induction Resistivity

Log type- Velocity

Logs available from 12230 feet to 15996 feet.

Curve type(s) available-

Self Potential

Acoustic Non-Compensated

Caliper

58. LOGDEX unique identification number- 129

Operator/Company- LONE STAR

Well name- H. A. FREDE 1

Location-Brazoria County, Texas

Township-Range-Section location- 8S-34E-1

Abstract/Tract/Block-A- 136

API number- 42- 39- 2791

HEADER data base unique number- 12208

Date of completion- 1/19/62

Well Classificattion code- D\&A

Log type- Induction Electrical

Logs available from 1986 feet to 10638 feet. 
Curve type(s) available-

Self Potential
Short Normal
Ampl ified Short Normal
Long Normal
Lateral
Induction Conductivity
Induction Resistivity

59. LOGDEX unique identification number- 130 Operator/Company - TEXACO

We 11 name- HOSKINS MOUND FEE NCT-1, 2

Location- Brazoria County, Texas

Township-Range-Section location- 8S-38E-2

Abstract/Tract/Block-A- 418

API number- 42- 39- 4913

HEADER data base unique number- 20356

Date of completion- 1/19/66

Well Classificattion code- GAS

Log type- Induction Electrical

Logs available from 100 feet to 17352 feet.

Curve type(s) available-

Self Potential

Short Norma 1

Amplified Short Normal

Induction Conductivity

Induction Resistivity

60. LOGDEX unique identification number- 132

Operator/Company- TENNECO

Well name- HAMMAN RANCH 1

Location- Hidalgo County, Texas 
Township-Range-Section location-29S-14E-1

API number- 42-215-

Log type- Velocity

Logs available from 5000 feet to 10008 feet.

Curve type(s) available-

Self Potential

Acoustic Non-Compensated

Caliper

61. LOGDEX unique identification number- 135

Operator/Company- PHILLIPS

We 11 name- HOUSTON "NN" 1

Location-Brazoria County, Texas

Township-Range-Section location- 6S-39E-7

Abstract/Tract/Block-A- 108

API number- 42- 39- 4887

HEADER data base unique number- 14992

Date of completion- 1/31/65

Well Classificattion code- D\&A

Log type- Induction Electrical

Logs available from 2500 feet to 15168 feet.

Curve type(s) available-

Self Potential

Short Normal

Ampl ified Short Normal

Induction Conductivity

Induction Resistivity

62. LOGDEX unique identification number- 136 
Summary of LOGDEX Data Base

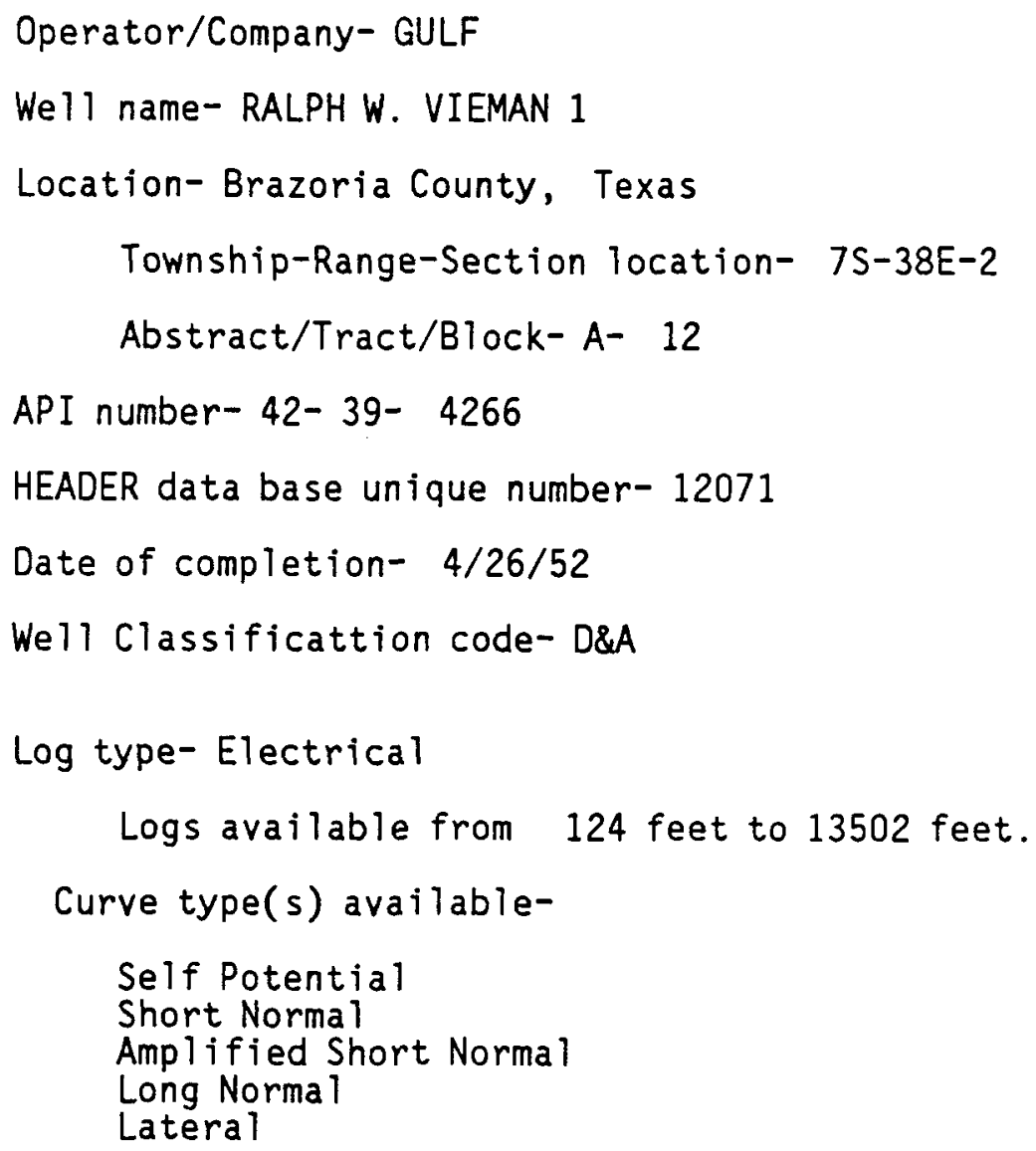

63. LOGDEX unique identification number- 137

Operator/Company- SLICK

Wel1 name- LYDIA CONKLIN 1

Location-Brazoria County, Texas

Township-Range-Section location- 6S-37E-8

API number- 42- 39-

Log type- Induction Electrical

Logs available from 1400 feet to 10300 feet.

Curve type(s) available-

Self Potential

Short Norma 1

Ampl if ied Short Normal 


\section{Induction Conductivity \\ Induction Resistivity}

64. LOGDEX unique identification number- 138

Operator/Company- HARGRAVE

We 11 name- FRED KLOBOUK 1

Location-Brazoria County, Texas

Township-Range-Section location- 5S-37E-7

Abstract/Tract/Block-A- 257

API number- 42- 39- 1388

HEADER data base unique number- 15674

Date of completion- 2/20/59

Well Classificattion code- D\&A

Log type- Induction Electrical

Logs available from 1600 feet to 9156 feet.

Curve type(s) available-

Self Potential

Short Normal

Amp lified Short Normal

Induction Conductivity

Induction Resistivity

65. LOGDEX unique identification number- 139

Operator/Company- SLICK

We 11 name- W. D. MUNSON 1

Location-Brazoria County, Texas

Township-Range-Section location- 7S-36E-7

Abstract/Tract/Block-A- 129

API number- $42-39-3848$ 
Summary of LOGDEX Data Base

\author{
HEADER data base unique number- 11348 \\ Date of completion- 5/29/60 \\ Well Classificattion code- D\&A \\ Log type- Induction Electrical \\ Logs available from 2472 feet to 11036 feet. \\ Curve type(s) available- \\ Self Potential \\ Short Normal \\ Amplified Short Normal \\ Induction Conductivity \\ Induction Resistivity
}

66. LOGDEX unique identification number- 140

Operator/Company- MID-CENTURY

Well name- F. W. HOWARD A-1

Location- Matagorda County, Texas

Township-Range-Section location- 7S-34E-4

API number- 42-321-

Log type- Induction Electrical

Logs available from 1530 feet to 11612 feet.

Curve type(s) available-

Self Potential

Short Normal

Ampl ified Short Normal

Induction Conductivity

Induction Resistivity

67. LOGDEX unique identification number- 141

Operator/Company- SOUTHWEST

Well name- MC DONALD 1

Page 50 


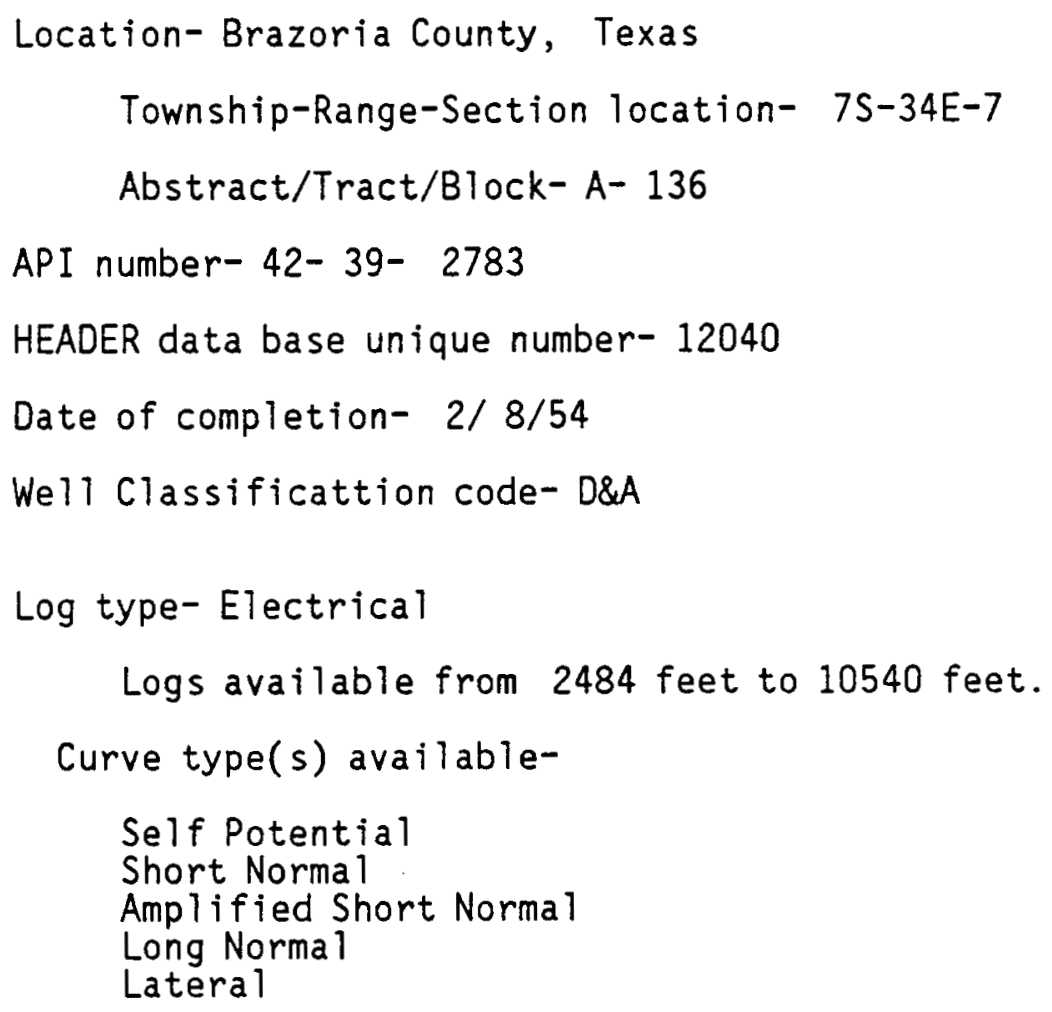

68. LOGDEX unique identification number- 142

Operator/Company- HUMBLE

Wel1 name-M. MC FARLAND 2

Location- Brazoria County, Texas

Township-Range-Section location- 7S-34E-8

Abstract/Tract/Block-A- 88

API number- 42- 39- 30255

HEADER data base unique number- 12039

Date of completion- $2 / 18 / 72$

Well Classificattion code- D\&A

Log type- Induction Electrical

Logs available from 2200 feet to 9056 feet.

Curve type(s) available- 


\author{
Self Potential \\ Induction Conductivity \\ Induction Resistivity \\ Focussed Resistivity \\ Amplified Focussed Resistivity
}

69. LOGDEX unique identification number- 143

Operator/Company- KIRBY PET AND RUSSELL MC FARLAND

We 11 name- K. N. GROCE 1

Location-Brazoria County, Texas

Township-Range-Section location- 7S-35E-1

Abstract/Tract/Block-A- 218

API number- 42- 39- 2852

HEADER data base unique number- 11347

Date of completion- $6 / 11 / 60$

Well Classificattion code- D\&A

Log type- Induction Electrical

Logs available from 2856 feet to 10802 feet.

Curve type(s) available-

Self Potential

Short Normal

Amplified Short Normal

Induction Conductivity

Induction Resistivity

70. LOGDEX unique identification number- 144

Operator/Company- PAN AMERICAN

We11 name- T. L. SMITH HEIRS 1

Location-Brazoria County, Texas

Township-Range-Section location- 7S-36E-3

Abstract/Tract/Block-A- 17

Page 52 


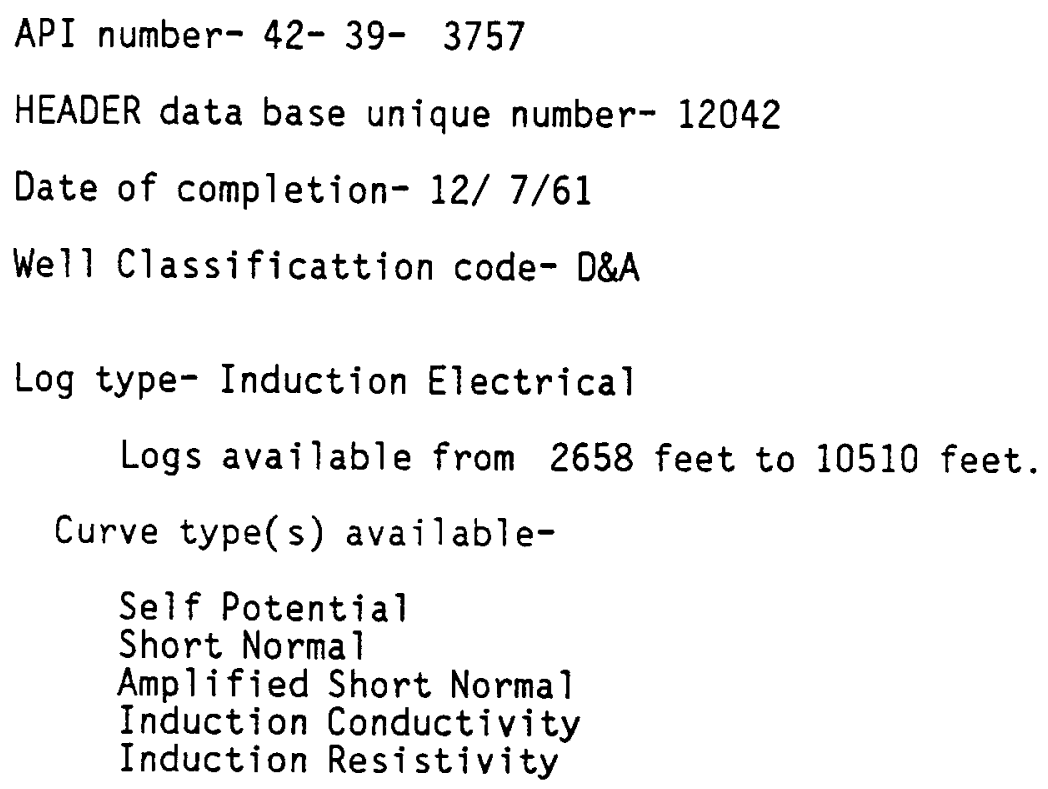

71. LOGDEX unique identification number- 145

Operator/Company- P. A. GALLERY

We 11 name- H. C. HUNSON A-1

Location-Brazoria County, Texas

Township-Range-Section location- 7S-36E-8

API number- 42- 39-

Log type- Electrical

Logs available from 2220 feet to 12502 feet.

Curve type(s) available-

Self Potential

Short Normal

Ampl ified Short Normal

Long Norma 1

Lateral

72. LOGDEX unique identification number- 146

Operator/Company- J. S. MICHAEL 
Summary of LOGDEX Data Base

\author{
Well name-W. N. MOORE 1 \\ Location- Brazoria County, Texas \\ Township-Range-Section location- 7S-37E-2 \\ Abstract/Tract/Block-A- 169 \\ API number- $42-39-4228$ \\ HEADER data base unique number- 12052 \\ Date of completion- $1 / 3 / 56$ \\ Well Classificattion code- D\&A \\ Log type- Electrical \\ Logs available from 2500 feet to 10974 feet. \\ Curve type(s) available- \\ Self Potential \\ Short Normal \\ Amplified Short Normal \\ Long Norma 1 \\ Lateral
}

73. LOGDEX unique identification number- 147

Operator/Company- PANO TECH EXPLORATION

We 11 name- JAMINSON 1

Location- Brazoria County, Texas

Township-Range-Section location- 7S-37E-6

Abstract/Tract/Block-A- 375

API number- 42- 39- 4190

HEADER data base unique number- 11349

Date of completion- $1 / 4 / 56$

Well Classificattion code- D\&A

Log type- Electrical

Logs available from 1396 feet to 14038 feet.

Page 54 
Curve type(s) available-

Self Potential

Short Norma 1

Ampl ified Short Normal

Long Norma 1

Lateral

74. LOGDEX unique identification number- 148

Operator/Company- CLEGG AND HUNT

Well name- G. C. CANNON 1

Location-Brazoria County, Texas

Township-Range-Section location- 7S-37E-1

Abstract/Tract/Block-A- 13

API number- 42- 39- 4230

HEADER data base unique number- 12054

Date of completion- $4 / 29 / 58$

Well Classificattion code- D\&A

Log type- Induction Electrical

Logs available from 3502 feet to 12060 feet.

Curve type(s) available-

Self Potential

Short Normal

Amplified Short Normal

Long Normal

Lateral

Induction Conductivity

Induction Resistivity

75. LOGDEX unique identification number- 149

Operator/Company- ROYAL RESOURCES

Wel1 name- M. W. METTLER TRUST 1

Location-Brazoria County, Texas 
Summary of LOGDEX Data Base

Township-Range-Section location- 7S-37E-1

Abstract/Tract/Block-A- 169

API number- 42- 39- 30060

HEADER data base unique number- 12056

Date of completion- 12/11/69

Well Classificattion code- D\&A

Log type- Induction Electrical

Logs available from 3500 feet to 11516 feet.

Curve type(s) available-
Self Potential
Short Norma]
Amplified Short Normal
Induction Conductivity
Induction Resistivity

76. LOGDEX unique identification number- 150

Operator/Company- PATRICK

Wel1 name- S. MOLLER 1

Location-Brazoria County, Texas

Township-Range-Section location- 7S-37E-1

API number- 42- 39-

Log type- Induction Electrical

Logs available from 794 feet to 10090 feet.

Curve type(s) available-

Self Potential

Short Normal

Ampl ified Short Normal

Induction Conductivity

Induction Resistivity 
77. LOGDEX unique identification number- 151

Operator/Company- ROWAN

Well name- NOVAK 1

Location-Brazoria County, Texas

Township-Range-Section location- 7S-38E-3

Abstract/Tract/Block-A- 14

API number- 42- 39- 5032

HEADER data base unique number- 12070

Date of completion- 11/15/67

Well Classificattion code- D\&A

Log type- Induction Electrical

Logs available from 2470 feet to 10562 feet.

Curve type(s) available-

Self Potential

Short Norma 1

Amplified Short Normal

Induction Conductivity

Induction Resistivity

78. LOGDEX unique identification number- 152

Operator/Company- ANSCHUTZ

We 11 name- H. L. PETERSON 1

Location- Brazoria County, Texas

Township-Range-Section location- 7S-38E-3

Abstract/Tract/Block-A- 13

API number- 42- 39- 30787

HEADER data base unique number- 12075

Date of completion- 6/5/77

Well Classificattion code- GAS 
Summary of LOGDEX Data Base

\author{
Log type- Induction Electrical \\ Logs available from 3250 feet to 13258 feet. \\ Curve type(s) available- \\ Self Potential \\ Induction Conductivity \\ Induction Resistivity \\ Focussed Resistivity \\ Amplified Focussed Resistivity
}

79. LOGDEX unique identification number- 153

Operator/Company- LARIO AND BELMONT

We 11 name-E. D. BIERI 1

Location-Brazoria County, Texas

Township-Range-Section location- 7S-38E-4

API number- 42- 39-

Log type- Induction Electrical

Logs available from 2116 feet to 12844 feet.

Curve type(s) available-

Self Potential

Short Normal

Amplified Short Normal

Induction Conductivity

Induction Resistivity

80. LOGDEX unique identification number- 154

Operator/Company- GENERAL CRUDE

Well name- T. MARTIN FEE 5

Location- Brazoria County, Texas

Township-Range-Section location- 7S-39E-1

API number- 42- 39-

Page 58 
Log type- Induction Electrical

Logs available from 12000 feet to 15172 feet.

Curve type(s) available-

Self Potential

Induction Conductivity

Focussed Resistivity

Amplified Focussed Resistivity

81. LOGDEX unique identification number- 155

Operator/Company-SUN

Well name- HOUSTON FARMS 1

Location-Brazoria County, Texas

Township-Range-Section location- 7S-39E-6

API number- 42- 39-

Log type-Electrical

Logs available from 382 feet to 18008 feet.

Curve type(s) available-

Self Potential

Short Normal

Amplified Short Normal

Long Norma 1

Lateral

82. LOGDEX unique identification number- 156

Operator/Company- GENERAL CRUDE

We 11 name- SHELL POINT 1

Location-Brazoria County, Texas

Township-Range-Section location- 7S-39E-6

API number- 42-39- 


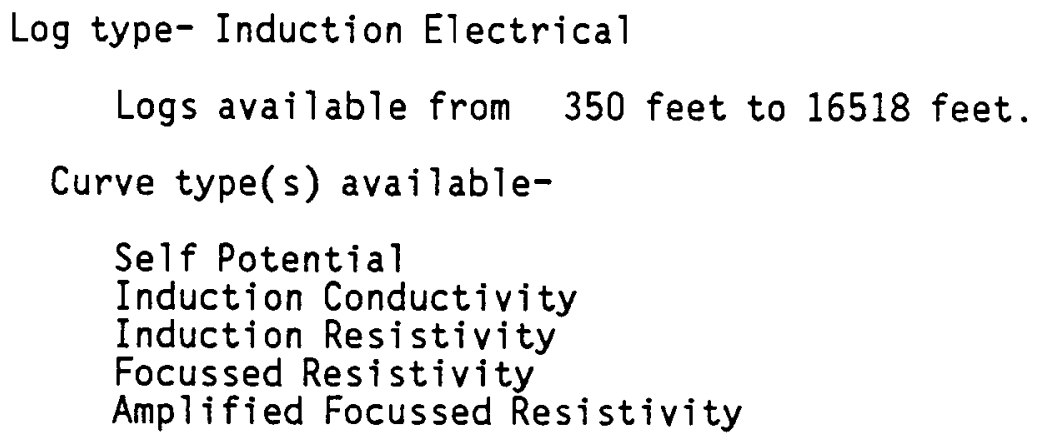

83. LOGDEX unique identification number- 157

Operator/Company- MC CULLOCH

Well name- LOBIT UNIT 1
Location-Galveston County, Texas
$\quad$ Township-Range-Section location- 7S-40E-1

API number- 42-167-

Log type- Induction Electrical

Logs available from 1480 feet to 18470 feet.

Curve type(s) available-

Self Potential

Induction Conductivity

Induction Resistivity

Focussed Resistivity

Amplified Focussed Resistivity

Gamma Ray API Units

84. LOGDEX unique identification number- 158

Operator/Company- PHILLIPS

Well name- HOUSTON "BB" 1

Location-Brazoria County, Texas

Township-Range-Section location- 7S-40E-4

API number- 42- 39-

Page 60 


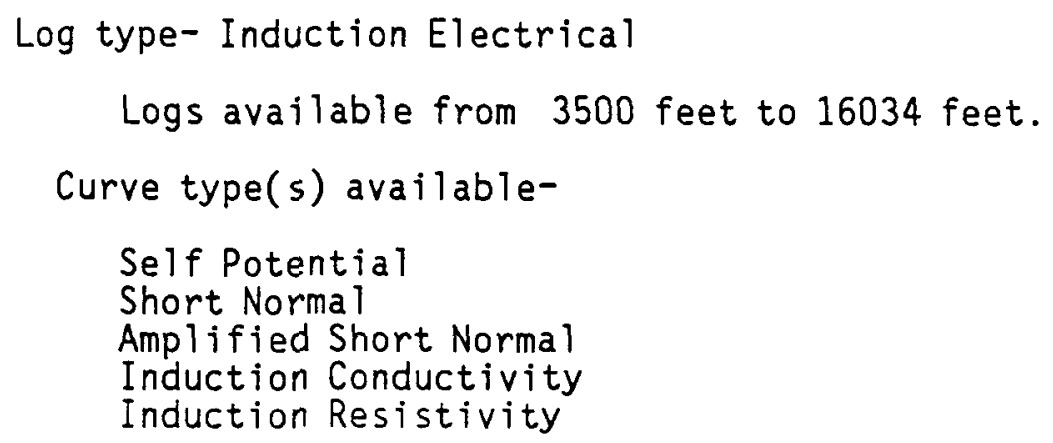

85. LOGDEX unique identification number- 159

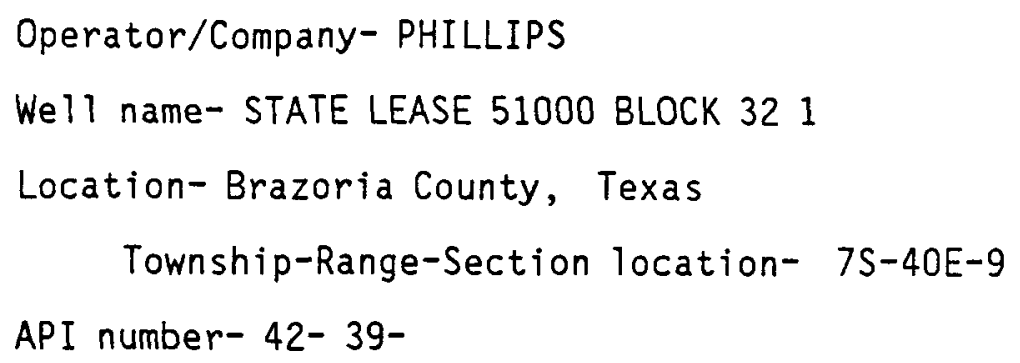

86. LOGDEX unique identification number- 160

Operator/Company- OIL AND GAS

Well name- BYRNE 1

Location- Fort Bend County, Texas 
Township-Range-Section location- 6S-34E-3

Abstract/Tract/Block-A- 33

API number- 42-157- 30193

HEADER data base unique number- 11901

Date of completion- $3 / 24 / 73$

Well Classificattion code- D\&A

Log type- Induction Electrical

Logs available from 520 feet to 7210 feet.

Curve type(s) available-

Self Potential

Short Norma 1

Amplified Short Normal

Induction Conductivity

Induction Resistivity

87. LOGDEX unique identification number- 161

Operator/Company- ROWAN ORILLING

We 11 name- KRAUSE 1

Location-Brazoria County, Texas

Township-Range-Section location- $6 S-34 E-8$

Abstract/Tract/B1ock-A- 120

API number- 42- 39- 4903

HEADER data base unique number- 11313

Date of completion- $7 / 7 / 66$

Well Classificattion code- D\&A

Log type- Induction Electrical

Logs available from 1380 feet to 9514 feet.

Curve type(s) available-

Self Potential

Short Norma 1

Page 62 


\begin{abstract}
Ampl ified Short Normal
Induction Conductivity

Induction Resistivity
\end{abstract}

88. LOGDEX unique identification number- 162

Operator/Company- PAN AMERICAN

Well name- N. W. HOPKINS 1

Location-Brazoria County, Texas

Township-Range-Section location- 6S-35E-4

API number- 42- 39-

Log type- Electrical

Logs available from 1174 feet to 6522 feet.

Curve type(s) available-

Self Potential

Short Normal

Ampl ifjed Short Normal

Long Norma 1

Lateral

89. LOGDEX unique identification number- 163

Operator/Company - DILLARD AND WALTERMIRE

Well name- CLOUDT 1

Location-Brazoria County, Texas

Township-Range-Section location- 6S-35E-8

Abstract/Tract/Block-A- 575

API number- 42- 39- 2020

HEADER data base unique number- 11314

Date of completion- $3 / 20 / 50$

Well Classificattion code- D\&A 
Summary of LOGDEX Data Base

Log type- Electrical

Logs available from 1900 feet to 10294 feet.

Curve type(s) available-

Self Potential

Short Norma 1

Amplified Short Normal

Long Norma 1

Lateral

90. LOGDEX unique identification number- 164

Operator/Company- SOUTHERN MINERALS

We 11 name- RAMSEY STATE FARM 1

Location-Brazoria County, Texas

Township-Range-Section location- 6S-36E-7

API number- 42- 39-

Log type- Induction Electrical

Logs available from 1864 feet to 10228 feet.

Curve type(s) available-

Self Potential

Short Normal

Amplified Short Normal

Induction Conductivity

Induction Resistivity

91. LOGDEX unique identification number- 165

Operator/Company- TEXAS

We 11 name- RAMSEY STATE FARM 1

Location-Brazoria County, Texas

Township-Range-Section location- 6S-37E-4

Abstract/Tract/Block-A- 25

Page 64 


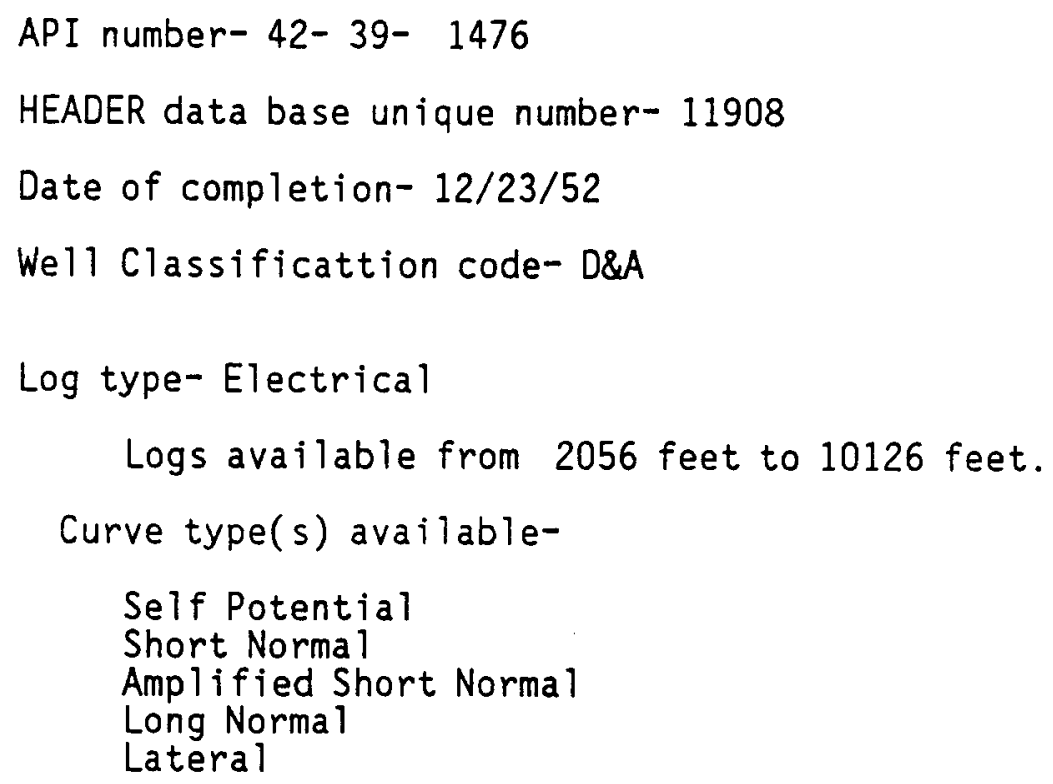

92. LOGDEX unique identification number- 166

Operator/Company- DAVIS

Well name- R. J. LOSTRACCO 1

Location-Brazoria County, Texas

Township-Range-Section location- 6S-37E-8

API number- 42- 39-

Log type- Induction Electrical

Logs available from 1320 feet to 10592 feet.

Curve type(s) available-

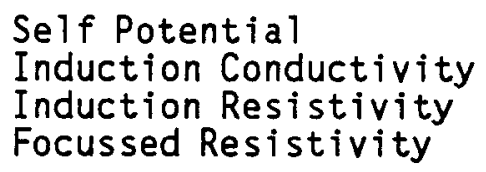

93. LOGOEX unique identification number- 167

Operator/Company- TEXAS 
Summary of LOGDEX Data Base

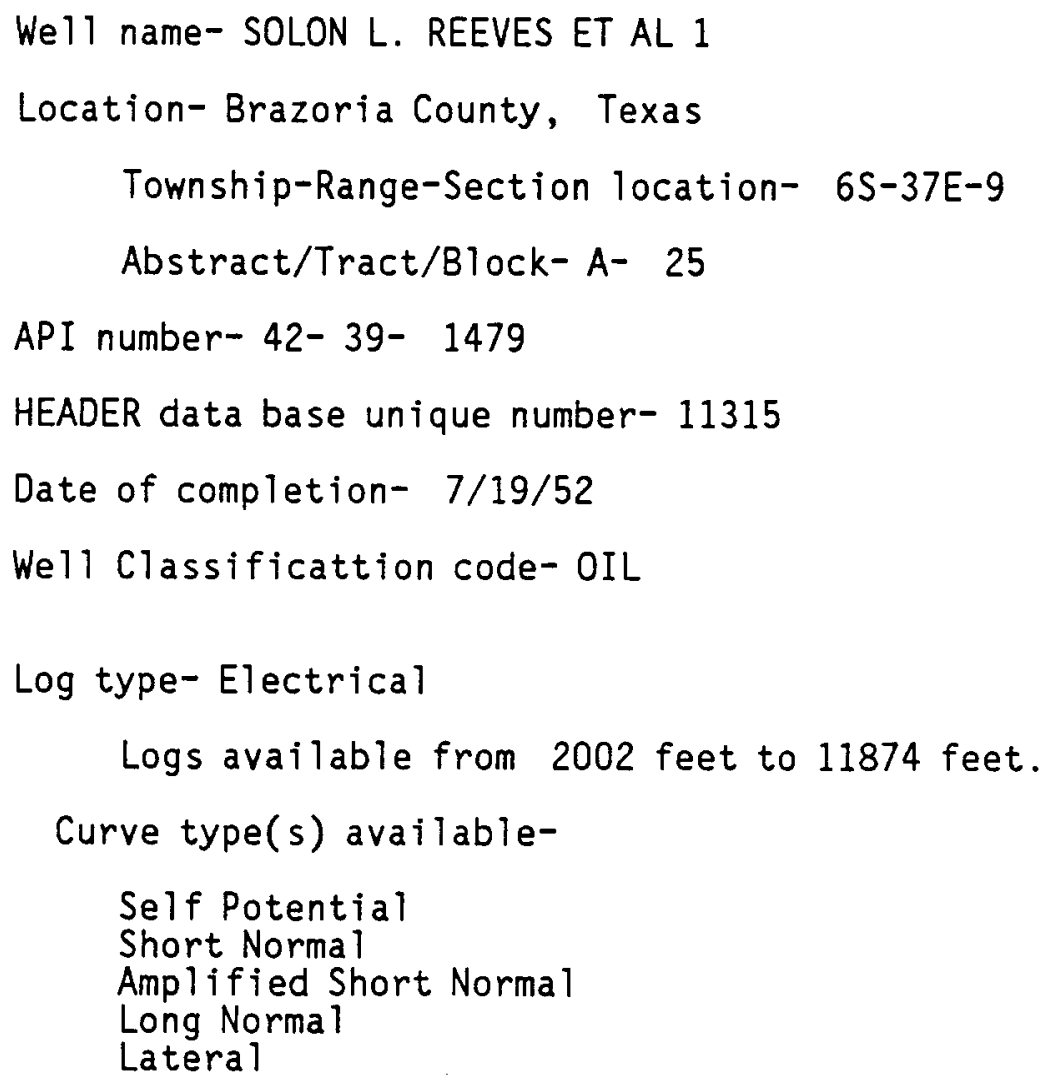

94. LOGDEX unique identification number- 168

Operator/Company- BROWN AND MC KENZIE

We 11 name- CLARK ESTATE 1

Location-Brazoria County, Texas

Township-Range-Section location- 6S-38E-1

Abstract/Tract/Block-A- 72

API number- 42- 39- 30383

HEADER data base unique number- 11910

Date of completion- 6/10/73

Well Classificattion code- D\&A

Log type- Induction Electrical

Logs available from 1600 feet to 11020 feet. 
Curve type(s) available-

Self Potential

Short Normal

Amplified Short Normal

Induction Conductivity

Induction Resistivity

95. LOGDEX unique identification number- 169

Operator/Company-ROWAN

We 11 name- GARRETT 1

Location-Brazoria County, Texas

Township-Range-Section location- 6S-38E-2

Abstract/Tract/Block- A- 100

API number- 42- 39- 1522

HEADER data base unique number- 11911

Date of completion-10/27/53

Well Classificattion code- D\&A

Log type- Electrical

Logs available from 990 feet to 12134 feet.

Curve type(s) available-

Self Potential

Short Normal

Ampl ified Short Normal

Long Norma 1

Lateral

96. LOGDEX unique identification number- 170

Operator/Company- MIDLAND PRODUCTION

Well name- E. W. WISSNER 1

Location-Brazoria County, Texas

Township-Range-Section location- 6S-38E-2 
Summary of LOGDEX Data Base

\author{
Abstract/Tract/Block-A- 100 \\ API number- 42- 39- 5110 \\ HEADER data base unique number- 11912 \\ Date of completion- $1 / 3 / 69$ \\ We 11 Classificattion code- D\&A \\ Log type- Induction Electrical \\ Logs available from 1490 feet to 10908 feet. \\ Curve type(s) available- \\ Self Potential \\ Short Normal \\ Amplified Short Normal \\ Induction Conductivity \\ Induction Resistivity
}

97. LOGDEX unique identification number- 171

Operator/Company- ADA

Well name- BAUGH 1

Location-Brazoria County, Texas

Township-Range-Section location- 6S-38E-6

Abstract/Tract/Block-A- 6

API number- 42- 39- 1570

HEADER data base unique number- 11918

Date of completion- 6/7/54

Well Classificattion code- GAS .

Log type- Electrical

Logs available from 2376 feet to 11648 feet.

Curve type(s) available-

Self Potential

Short Normal

Amplified Short Normal

Long Norma 1

Page 68 


\section{Lateral}

98. LOGDEX unique identification number- 172

Operator/Company- PHILLIPS

We 11 name- HOUSTON "S" 1

Location-Brazoria County, Texas

Township-Range-Section location- 6S-39E-5

Abstract/Tract/Block-A- 110

API number- 42- 39- 986

HEADER data base unique number- 14981

Date of completion- $2 / 27 / 52$

Well Classificattion code- GAS

Log type- Micro

Logs available from 9192 feet to 15000 feet.

Curve type(s) available-

Self Potential

Inverse 1 Inch

99. LOGDEX unique identification number- 173

Operator/Company- PHILLIPS

We 11 name- HOUSTON "F" 3

Location-Brazoria County, Texas

Township-Range-Section location- 6S-39E-7

API number- 42- 39-

Log type- Micro

Logs available from 8936 feet to 12458 feet. 
Summary of LOGDEX Data Base

Curve type(s) available-

Self Potential

Inverse 1 Inch

Caliper

100. LOGDEX unique identification number- 174

Operator/Company- PHILLIPS

We 11 name- CHRISTENSEN A-1

Location-Galveston County, Texas

Township-Range-Section location- 6S-40E-4

API number- 42-167-

Log type- Micro

Logs available from 9330 feet to 15508 feet.

Curve type(s) available-

Self Potential

Inverse 1 Inch

Caliper

101. LOGDEX unique identification number- 175

Operator/Company- PHILLIPS

We 11 name- LAUZON 1

Location-Galveston County, Texas

Township-Range-Section location- 6S-40E-4

Abstract/Tract/Block-A- 47

API number- 42-167- 1441

HEADER data base unique number- 15036

Date of completion- 10/19/60

Well Classificattion code- D\&A 
Log type- Micro

Logs available from 8898 feet to 12512 feet.

Curve type(s) available-

Self Potential

Inverse 1 Inch

Caliper

102. LOGDEX unique identification number- 176

Operator/Company- HASSIE HUNT TRUST

We11 name- HORTON SAYKO ET AL 2

Location-Galveston County, Texas

Township-Range-Section location- 6S-40E-5

Abstract/Tract/Block-A- 172

API number- 42-167- 1450

HEADER data base unique number- 11962

Date of completion- $10 / 12 / 58$

We11 Classificattion code- M-G

Log type-Micro

Logs available from 9300 feet to 11890 feet.

Curve type(s) available-

Self Potential

Inverse 1 Inch

Caliper

103. LOGDEX unique identification number- 177

Operator/Company- HASSIE HUNT TRUST

We 11 name- A. TACQUARD ET AL 1

Location-Galveston County, Texas

Township-Range-Section-1ocation- 6S-40E-5 
Summary of LOGDEX Data Base

\author{
Abstract/Tract/Block-A- 337 \\ API number- 42-167- 30486 \\ HEADER data base unique number- 15034 \\ Date of completion- $12 / 27 / 60$ \\ Well Classificattion code- GAS \\ Log type- Micro \\ Logs available from 9000 feet to 13250 feet. \\ Curve type(s) available- \\ Self Potential \\ Inverse 1 Inch \\ Caliper
}

104. LOGDEX unique identification number- 178

Operator/Company- BREWSTER AND BARTLE

We 11 name- UNIVERSITY OF TEXAS B-1

Location-Galveston County, Texas

Township-Range-Section location- 6S-41E-1

API number- 42-167-

Log type- Induction Electrical

Logs available from 5020 feet to 11734 feet.

Curve type(s) available-

Self Potential

Short Norma 1

Amplified Short Normal

Induction Conductivity

Induction Resistivity

105. LOGDEX unique identification number- 179

Operator/Company- JOHN W. MECOM

Page 72 


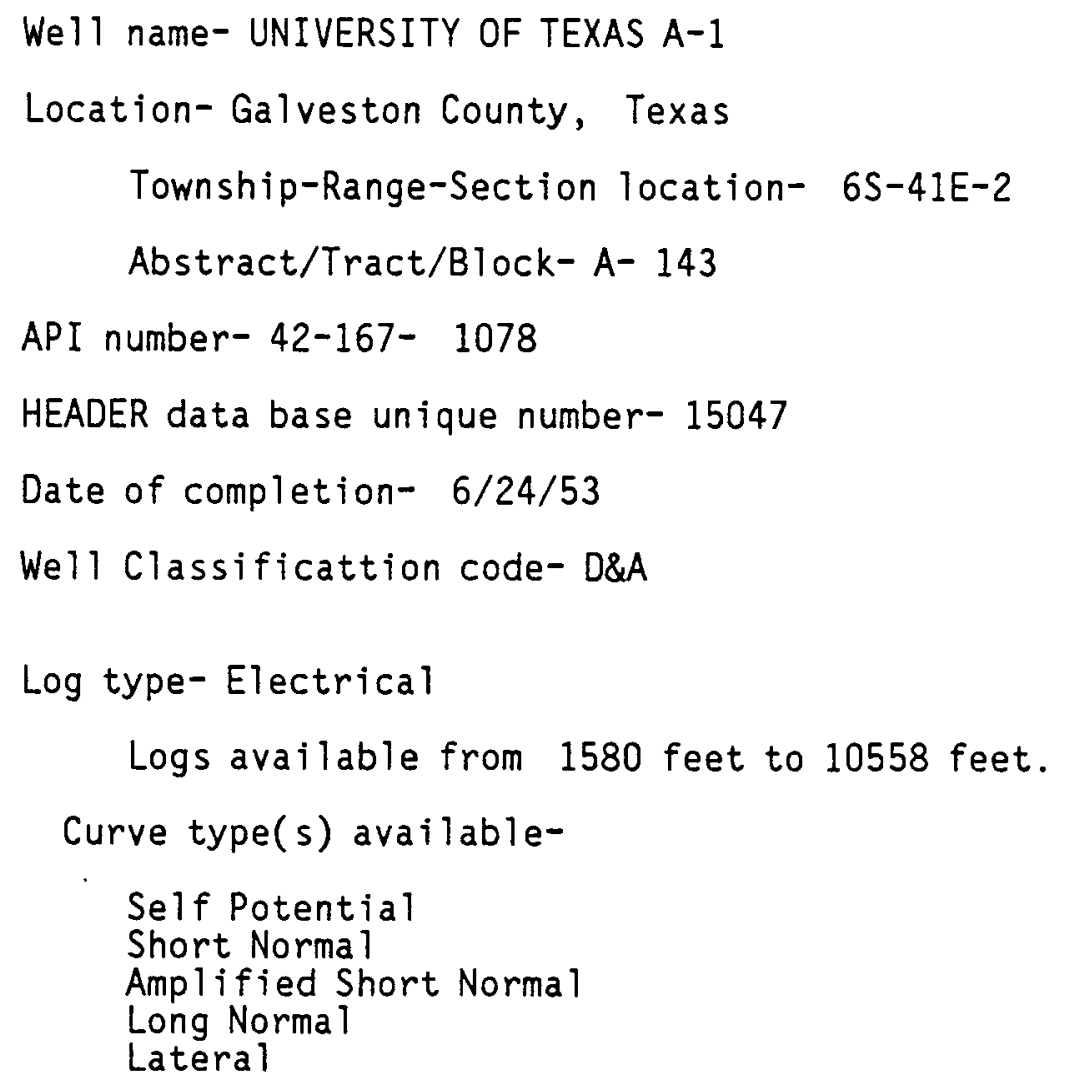

106. LOGDEX unique identification number- 180

Operator/Company- DORCHESTER EXPLORATION

We 11 name- UNIVERSITY OF TEXAS 1

Location-Galveston County, Texas

Township-Range-Section location- 6S-41E-2

Abstract/Tract/Block-A- 71

API number- 42-167- 177

HEADER data base unique number- 15046

Date of completion- 10/26/63

Well Classificattion code- GAS

Log type- Induction Electrical

Logs available from 1232 feet to 10986 feet. 
Curve type(s) available-

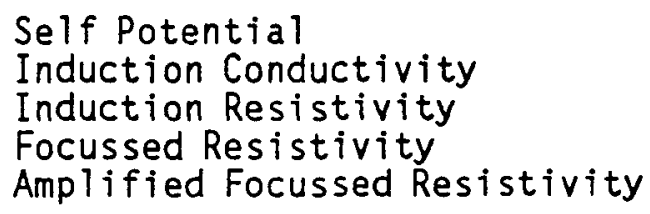

107. LOGDEX unique identification number- 181

Operator/Company- MID STATES

We11 name- WESTERLAGE UNIT 1

Location-Galveston County, Texas

Township-Range-Section location- 6S-41E-3

Abstract/Tract/Block-A- 150

API number- 42-167- 1142

HEADER data base unique number- 15049

Date of completion- $11 / 8 / 52$

Well Classificattion code- D\&A

Log type- Electrical

Logs available from 88 feet to 9854 feet.

Curve type(s) available-

Self Potential

Short Normal

Amplified Short Normal

Long Norma 1

Latera 1

108. LOGDEX unique identification number- 182

Operator/Company- HUMBLE

Well name-W. H. COON FEE 1

Location-Galveston County, Texas

Township-Range-Section location- 6S-41E-3 


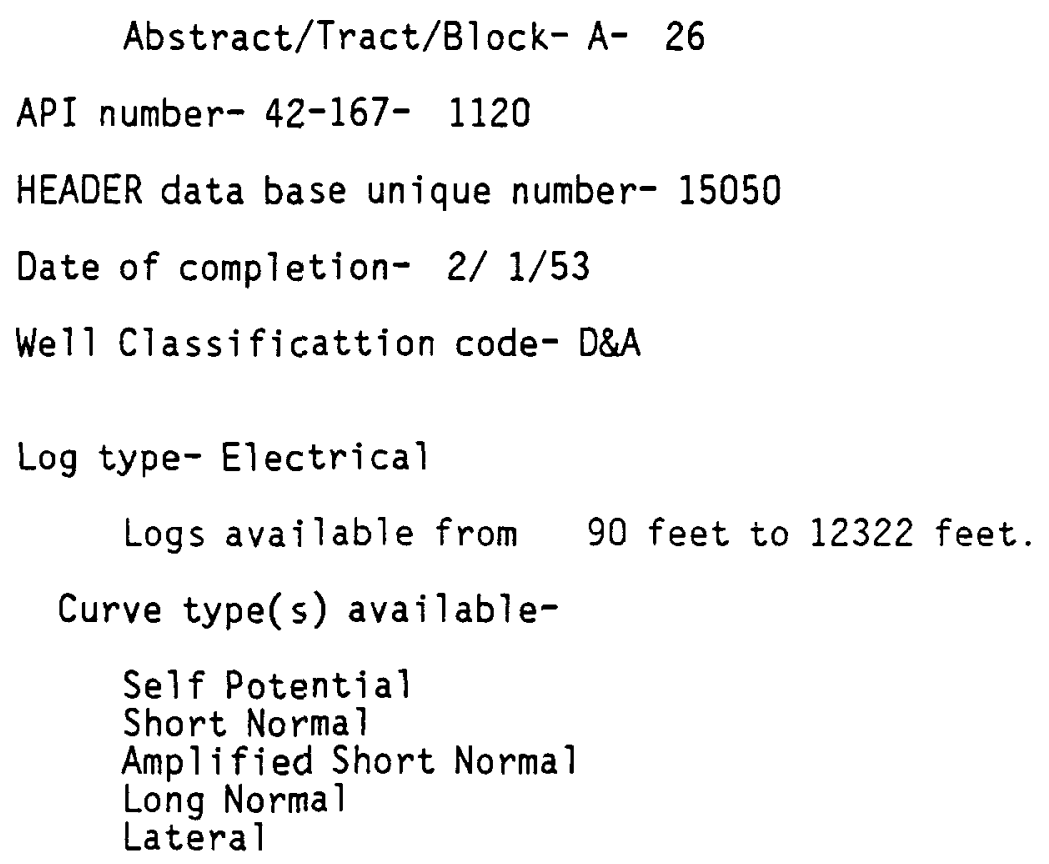

109. LOGDEX unique identification number- 183

Operator/Company-JOCELYN-VARN, COLORADO, JADE

Well name- PIERCE ESTATE 1

Location-Galveston County, Texas

Township-Range-Section location- 6S-41E-4

Abstract/Tract/Block- A- 175

API number- 42-167- 1080

HEADER data base unique number- 15048

Date of completion- 7/10/62

Well Classificattion code- D\&A.

Log type- Induction Electrical

Logs available from 480 feet to 11526 feet.

Curve type(s) available-

Self Potential

Short Normal

Amplified Short Normal

Induction Conductivity 
Summary of LOGDEX Data Base

$$
\text { Induction Resistivity }
$$

110. LOGDEX unique identification number- 184

Operator/Company- GULF

Well name- BOLIVAR TRACT "A" 1

Location-Galveston County, Texas

Township-Range-Section location- 6S-42E-1

API number- 42-167-

Log type- Velocity

Logs available from 8454 feet to 13988 feet.

Curve type(s) available-

Self Potential

Acoustic Compensated

Caliper

111. LOGDEX unique identification number- 185

Operator/Company- GULF

We11 name- FEDERAL PELICAN 1

Location- Galveston County, Texas

Township-Range-Section location- 6S-42E-2

Abstract/Tract/Block- T- 133

API number- 42-167- 2037

HEADER data base unique number- 15045

Date of completion- 5/21/67

Well Classificattion code- D\&A

Log type- Velocity

Logs available from 4972 feet to 13380 feet. 
Curve type(s) available-

Self Potential

Acoustic Compensated

Caliper

Log type- Induction Electrical

Logs available from 2560 feet to 13390 feet.

Curve type(s) available-

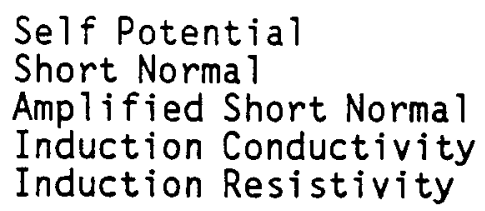

112. LOGDEX unique identification number- 186

Operator/Company- GULF

Well name- COLINE 1

Location-Galveston County, Texas

Township-Range-Section location- 6S-42E-1

Abstraçt/Tract/Block-A- 162

API number- 42-167- 2012

HEADER data base unique number- 15044

Date of completion- 2/12/65

Well Classificattion code- GAS

Log type- Induction Electrical

Logs available from 160 feet to 15562 feet.

Curve type(s) available-

Self Potential

Short Normal

Amplified Short Normal

Induction Conductivity

Induction Resistivity 
Summary of LOGDEX Data Base

113. LOGDEX unique identification number- 187

Operator/Company- BREWSTER-BARTLE

We 11 name- $100 \mathrm{~A}-1$

Location-Galveston County, Texas

Township-Range-Section location- $6 S-42 E-3$

Abstract/Tract/Block- T- 100

API number- 42-167- 1067

HEADER data base unique number- 15030

Date of completion- $11 / 6 / 62$

Well Classificattion code- D\&A

Log type- Induction Electrical

Logs available from 2376 feet to 11758 feet.

Curve type(s) available-

Self Potential

Short Normal

Amplified Short Normal

Induction Conductivity

Induction Resistivity

114. LOGDEX unique identification number- 188

Operator/Company- GEORGE MITCHELL \& ASSOC.

We 11 name- GALVESTON TOWNSIDE UNIT 21

Location-Galveston County, Texas

Township-Range-Section location- 6S-42E-5

API number- 42-167-

Log type- Induction Electrical

Logs available from 2550 feet to 13032 feet. 
Curve type(s) available-

Self Potential

Short Normal

Amplified Short Normal

Induction Conductivity

Induction Resistivity

115. LOGDEX unique identification number- 189

Operator/Company-SCURLOCK AND M. T. HALBOUTY

We 11 name- DENNIS KRAUSE 1

Location-Fort Bend County, Texas

Township-Range-Section location- 5S-33E-2

Abstract/Tract/Block-A- 143

API number- 42-157- 3052

HEADER data base unique number- 15059

Date of completion- 8/27/63

Well Classificattion code- GAS

Log type- Induction Electrical

Logs available from 1048 feet to 8330 feet.

Curve type(s) available-

Self Potential

Short Normal

Amplified Short Normal

Induction Conductivity

Induction Resistivity

116. LOGDEX unique identification number- 190

Operator/Company- FORT BEND

Wel1 name- J. M. MOORE EST. 1

Location-Fort Bend County, Texas

Township-Range-Section location- 5S-33E-8 
Summary of LOGDEX Data Base

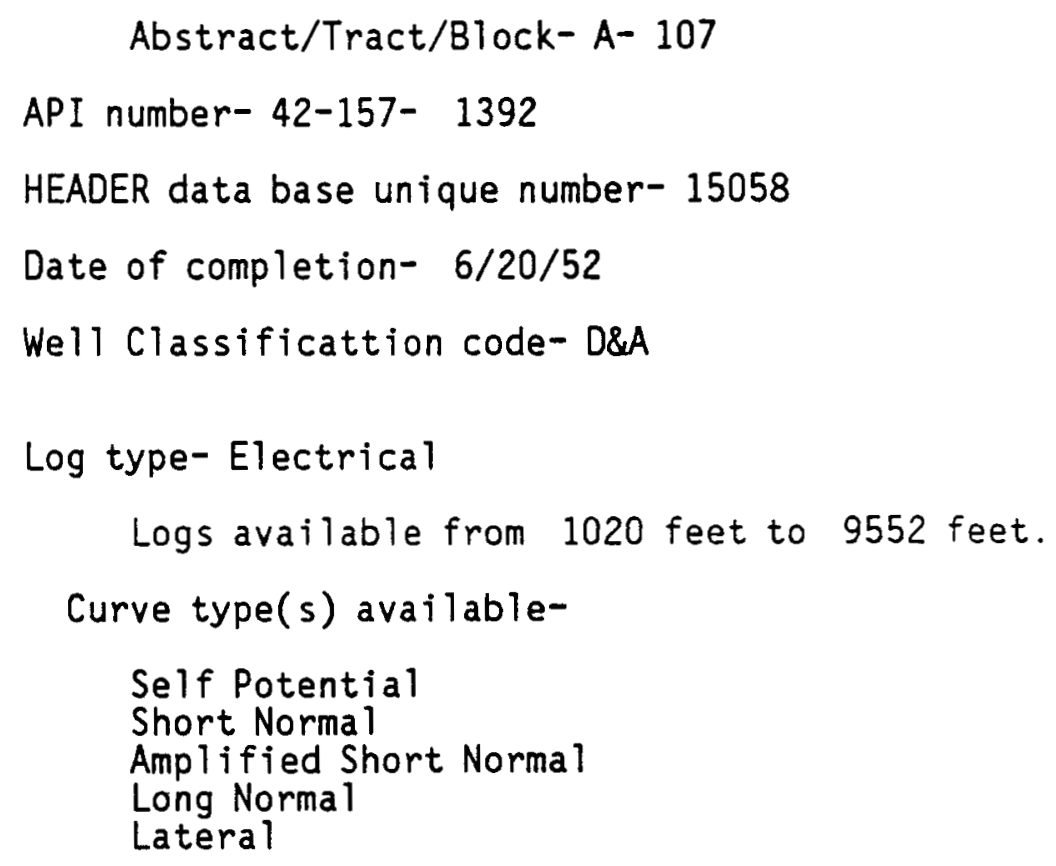

117. LOGDEX unique identification number- 191

Operator/Company-WINDWARD AND H. B. OWNBY

Well name- F. W. SIMS 1

Location- Fort Bend County, Texas

Township-Range-Section location- 5S-34E-3

API number- 42-157-

Log type- Electrical

Logs available from 1000 feet to 6048 feet.

Curve type(s) available-

Self Potential

Short Normal

118. LOGDEX unique identification number- 192 Operator/Company- ATLANTIC REFINING 
We11 name- JULIUS H. GURRELS 1

Location- Fort Bend County, Texas

Township-Range-Section location- 5S-35E-6

Abstract/Tract/Block- A- 303

API number- 42-157- 1978

HEADER data base unique number- 15054

Date of completion-10/30/63

Well Classificattion code- $M-G$

Log type- Induction Electrical

Logs available from 2040 feet to 8240 feet.

Curve type(s) available-

Self Potential

Short Norma 1

Ampl ified Short Normal

Induction Conductivity

Induction Resistivity

119. LOGDEX unique identification number- 193

Operator/Company-SLADE

Well name- SALLIE BROWN KENNELY 1

Location- Fort Bend County, Texas

Township-Range-Section location- 5S-35E-7

Abstract/Tract/Block-A- 8

API number- 42-157- 1992

HEADER data base unique number- 15053

Date of completion- $8 / 30 / 61$

Well Classificattion code- D\&A

Log type- Induction Electrical

Logs available from 1496 feet to 9666 feet. 
Summary of LOGDEX Data Base

Curve type(s) available-

Self Potential

Short Normal

Ampl ified Short Normal

Induction Conductivity

Induction Resistivity

120. LOGDEX unique identification number- 194

Operator/Company- BECK

We 11 name- SARA WEST HEIRS ET. AL. UNIT \#1 1

Location- Brazoria County, Texas

Township-Range-Section location- 5S-36E-8

API number- 42- 39-

Log type- Induction Electrical

Logs available from 820 feet to 8270 feet.

Curve type(s) available-

Self Potential

Short Normal

121. LOGDEX unique identification number- 195

Operator/Company- J. K. DORRANCE

Well name- J. E. FOSTER 1

Location-Fort Bend County, Texas

Township-Range-Section location- 5S-37E-4

Abstract/Tract/Block-A- 68

API number- 42-157- 2779

HEADER data base unique number- 18195

Date of completion- $7 / 17 / 53$

Well Classificattion code- D\&A 


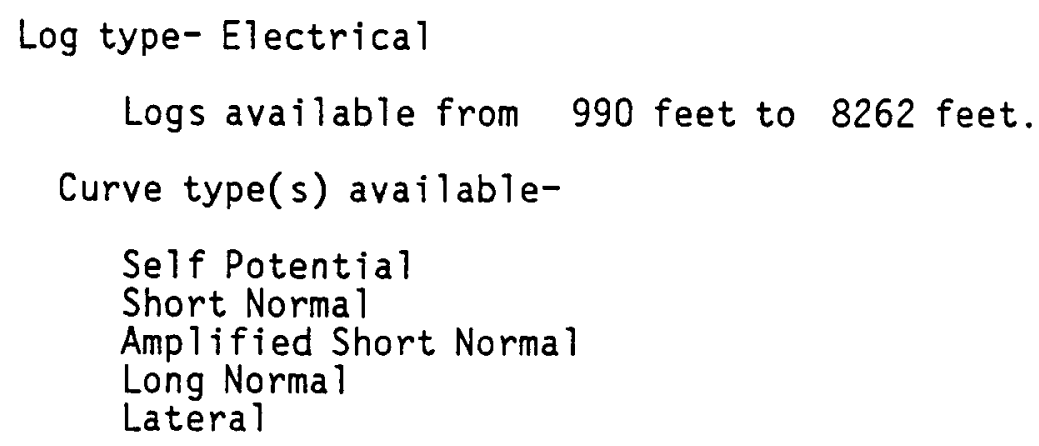

122. LOGDEX unique identification number- 196

Operator/Company- HUMBLE AND JACK FRAZIER

Well name- HUMBLE UNIT 1

Location-Brazoria County, Texas

Township-Range-Section location- 5S-37E-6

Abstract/Tract/Block-A- 517

API number- 42- 39- 1354

HEADER data base unique number- 15672

Date of completion- $12 / 29 / 48$

Well Classificattion code- D\&A

Log type- Electrical

Logs available from 1790 feet to 9626 feet.

Curve type(s) available-

Self Potential

Short Norma 1

Ampl ified Short Normal

Long Normal

Lateral

123. LOGDEX unique identification number- 197

Operator/Company- TEXAS CITY REFINING

We 11 name- SHARP CORPORATION 1 
Summary of LOGDEX Data Base

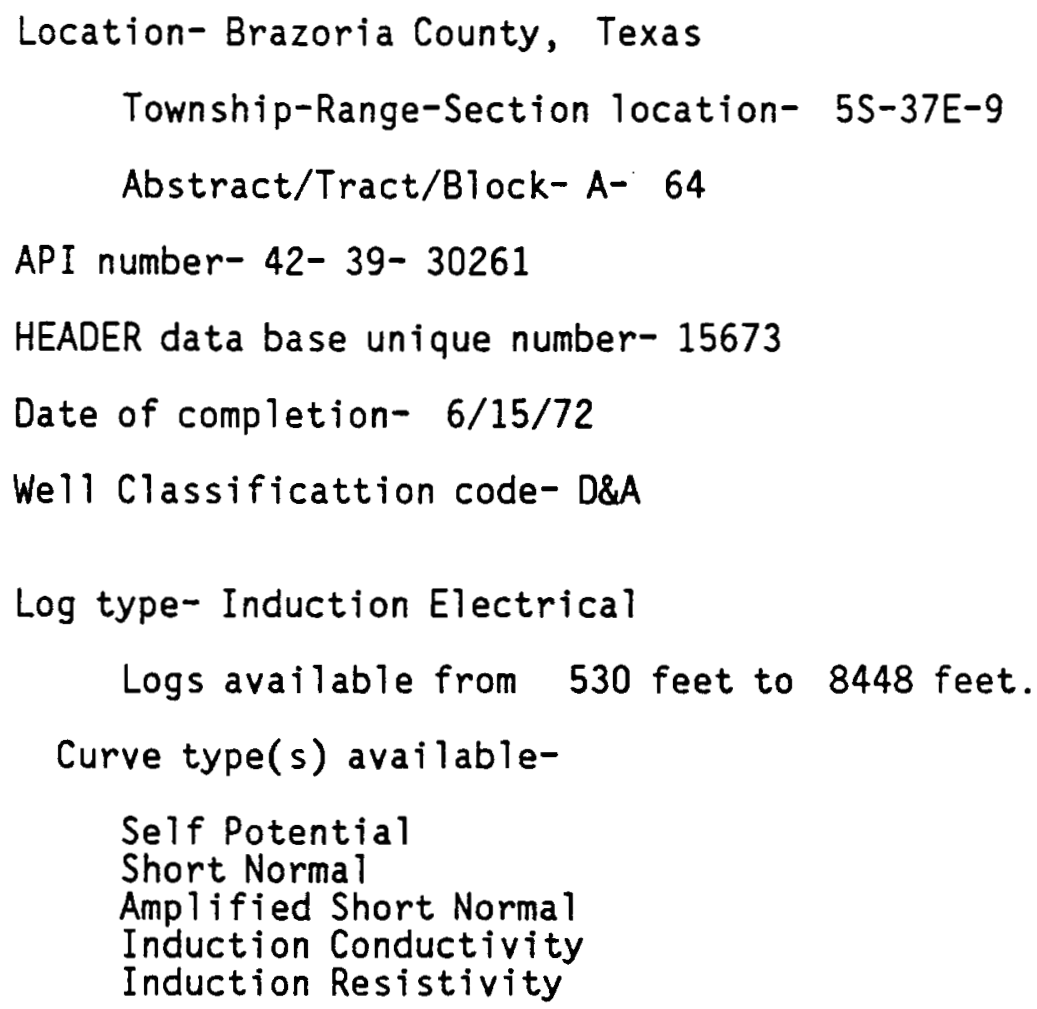

124. LOGDEX unique identification number- 198

Ọperator/Company- TEXAS AND FORT BEND

We 11 name- HOUSTON FARMS DEV CO. 2

Location-Brazoria County, Texas

Township-Range-Section location- 6S-39E-8

API number- 42- 39-

Log type- Electrical

Logs available from 1526 feet to 15524 feet.

Curve type(s) available-

Self Potential

Short Normal

Ampl if ied Short Normal

Long Normal

Lateral 
125. LOGDEX unique identification number- 199

Operator/Company- J. S. MICHAEL

We 11 name- LEEDY 1

Location-Brazoria County, Texas

Township-Range-Section location- 5S-38E-4

Abstract/Tract/Block-A- 359

API number- 42- 39- 1198

HEADER data base unique number- 11778

Date of completion-11/27/53

Well Classificattion code- D\&A

Log type-Electrical

Logs available from 1250 feet to 8820 feet.

Curve type(s) available-

Self Potential

Short Normal

Amplified Short Normal

Long Normal

Lateral

126. LOGDEX unique identification number- 200

Operator/Company- SKELLY

We11 name- A. W. ADAM 1

Location-Brazoria County, Texas

Township-Range-Section location- 5S-38E-9

API number- 42-39-

Log type- Electrical

Logs available from 1626 feet to 9610 feet. 
Summary of LOGDEX Data Base

\author{
Curve type(s) available- \\ Self Potential \\ Short Normal \\ Ampl ified Short Normal \\ Long Norma 1 \\ Lateral
}

127. LOGDEX unique identification number- 201

Operator/Company- PATRICK R. RUTHERFORD

Well name-F. K. MILLER 1

Location-Galveston County, Texas

Township-Range-Section location- 5S-39E-1

Abstract/Tract/Block-A- 9

API number- 42-167- 1274

HEADER data base unique number- 11780

Date of completion- $8 / 20 / 57$

Well Classificattion code- D\&A

Log type- Induction Electrical

Logs available from 2300 feet to 10014 feet.

Curve type(s) available-

Self Potential

Short Normal

Amplified Short Normal

Long Normal

Lateral

Induction Conductivity

Induction Resistivity

128. LOGDEX unique identification number- 202

Operator/Company- PAN AMERICAN AND WESLEY WEST

Well name- JOCKUSCH OIL UNIT 1

Location-Galveston County, Texas

Page 86 


\section{Township-Range-Section location- 5S-39E-2 \\ Abstract/Tract/Block-A- 609}

API number- 42-167- 2083

HEADER data base unique number- 11782

Date of completion- $1 / 7 / 68$

Well Classificattion code- D\&A

Log type- Induction Electrical

Logs available from 100 feet to 10170 feet.

Curve type(s) available-

Self Potential

Short Normal

Amp lified Short Normal

Induction Conductivity

Induction Resistivity

129. LOGDEX unique identification number- 203

Operator/Company- SUPERIOR AND PAN AMERICAN

Well name- WINTON UNIT 1

Location-Galveston County, Texas

Township-Range-Section location- 5S-39E-8

Abstract/Tract/Block-A- 324

API number- 42-167- 1125

HEADER data base unique number- 12839

Date of completion- 12/20/57

Well Classificattion code- GAS

Log type- Induction Electrical

Logs available from 1900 feet to 13524 feet.

Curve type(s) available-

Self Potential

Short Normal 


$$
\begin{aligned}
& \text { Amplified Short Normal } \\
& \text { Induction Conductivity } \\
& \text { Induction Resistivity }
\end{aligned}
$$

130. LOGDEX unique identification number- 204

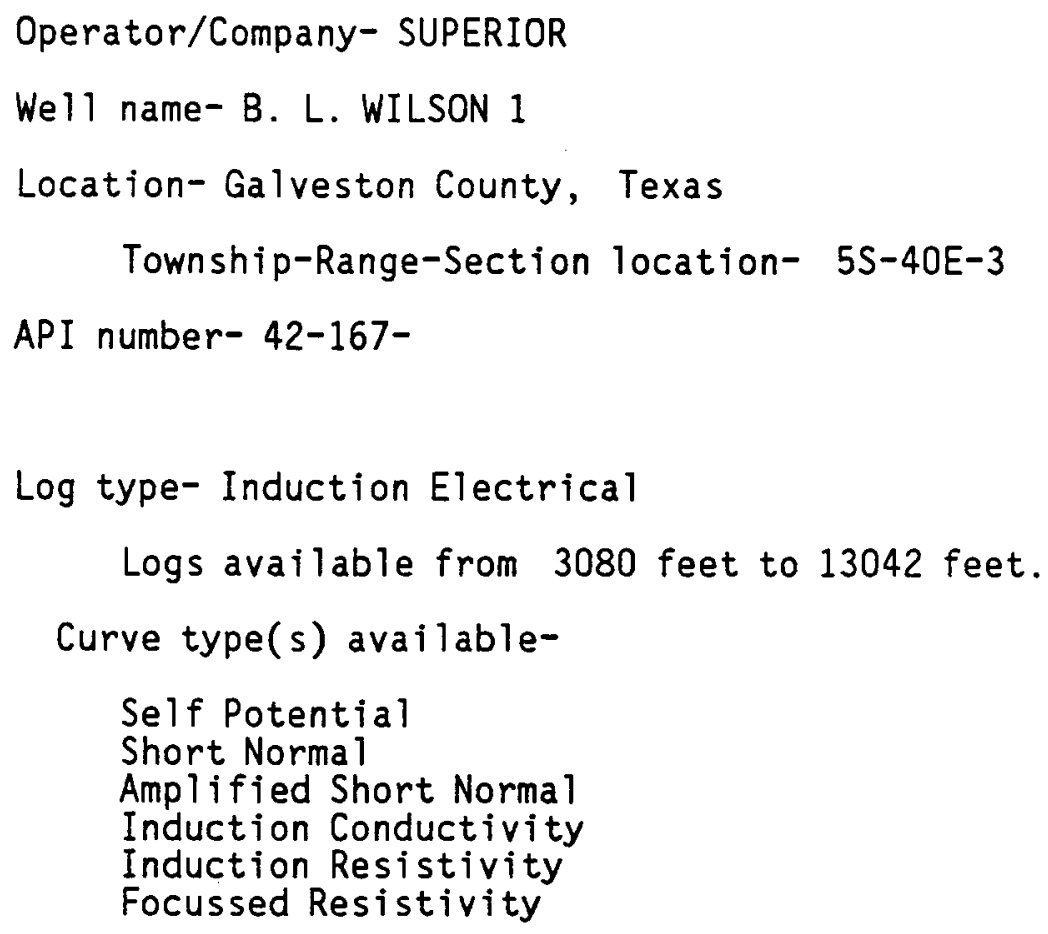

131. LOGDEX unique identification number- 205

Operator/Company- CITIES SERVICE

Well name- STEWART B-2

Location-Galveston County, Texas:

Township-Range-Section location- 5S-40E-4

API number- 42-167-

\author{
Log type- Induction Electrical \\ Logs available from 3028 feet to 14930 feet. \\ Curve type(s) available-
}

Page 88 
Self Potential

Short Normal

Amplified Short Normal

Induction Conductivity

132. LOGDEX unique identification number- 206

Operator/Company- JOHN W. MECON ET AL

We 11 name- ERVINE E. BISHOP 5

Location-Galveston County, Texas

Township-Range-Section location- 5S-40E-5

API number- 42-167-

Log type- Electrical

Logs available from 2960 feet to 14172 feet.

Curve type(s) available-

Self Potential

Short Normal

Ampl ified Short Normal

Long Normal

Lateral

133. LOGDEX unique identification number- 207

Operator/Company- JOHN W. MECON

Well name- IRVINE \& BISHOP 2

Location-Galveston County, Texas

Township-Range-Section location- 5S-40E-6

Abstract/Tract/Block-A- 208

API number- 42-167- 1623

HEADER data base unique number- 20404

Date of completion- $2 / 6 / 51$

We11 Classificattion code- D\&A 
Summary of LOGDEX Data Base

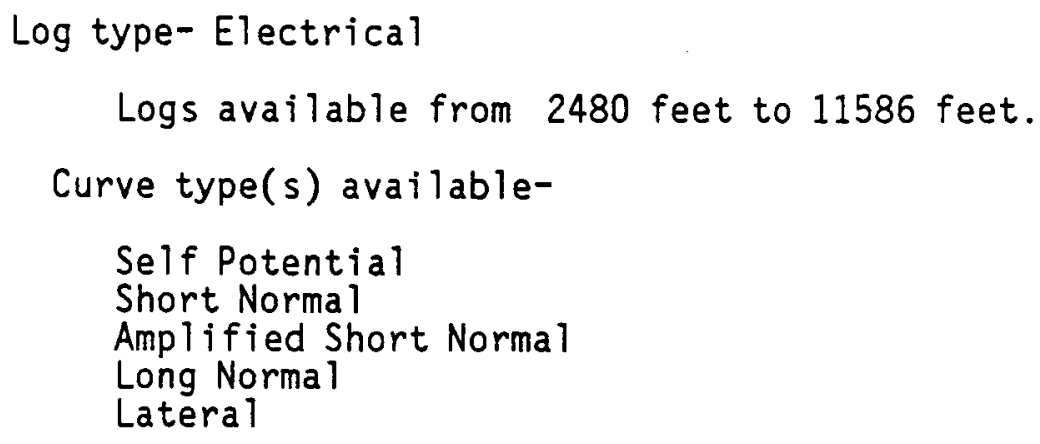

134. LOGDEX unique identification number- 208

Operator/Company- JOHN W. MECON

Well name- ERVINE-BISHOP 4

Location- Gaiveston County, Texas

Township-Range-Section location- 5S-40E-8

Abstract/Tract/Block- A- 552

API number- 42-167- 1266

HEADER data base unique number- 11789

Date of completion- 11/27/61

Well Classificattion code- GAS

Log type- Electrical

Logs available from 1310 feet to 15050 feet.

Curve type(s) available-

Self Potential

Short Normal

Ampl if ied Short Normal

Long Normal

Lateral

135. LOGDEX unique identification number- 209

Operator/Company- ROWAN AND TEXAS GULF

Well name-MRS. CORINE SCOTT 1

Page 90 


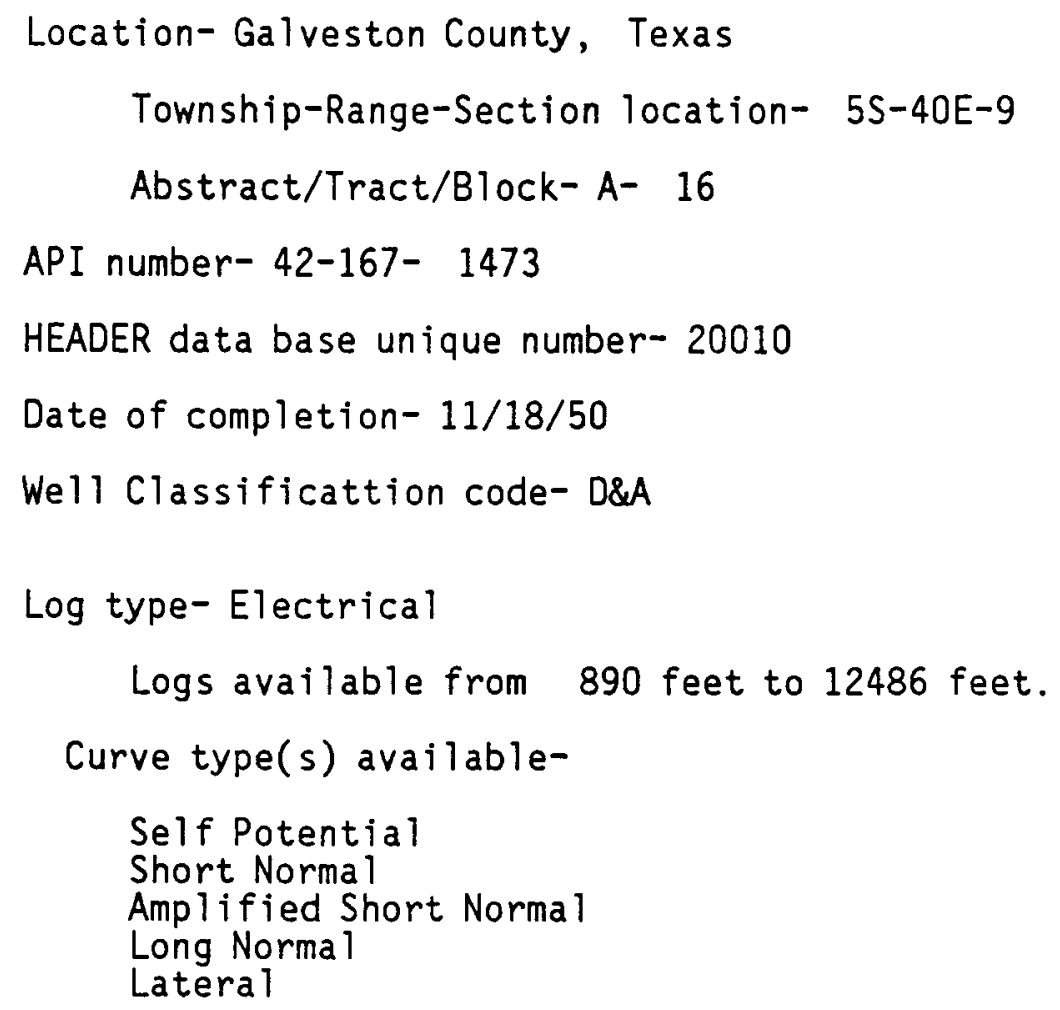

136. LOGDEX unique identification number- 210

Operator/Company- UNION

Well name- 3101

Location-Galveston County, Texas

Township-Range-Section location- 5S-41E-1

Abstract/Tract/Block- T- 310

API number- 42-167- 962

HEADER data base unique number- 11.795

Date of completion- $3 / 2 / 62$

Well Classificattion code- D\&A

Log type- Induction Electrical

Logs available from 2500 feet to 11732 feet.

Curve type(s) available- 


\author{
Self Potential \\ Short Normal \\ Amplified Short Normal \\ Induction Conductivity \\ Induction Resistivity
}

137. LOGDEX unique identification number- 211

Operator/Company- PAN AMERICAN

We 11 name- HOUSTON NEGRO HOSPITAL I

Location- Galveston County, Texas

Township-Range-Section location- 5S-41E-3

API number- 42-167-

Log type- Electrical

Logs available from 1526 feet to 12078 feet.

Curve type(s) available-

Self Potential

Short Normal

Ampl ified Short Normal

Long Normal

Lateral

138. LOGDEX unique identification number- 212

Operator/Company- HUMBLE

We 11 name- BAYOU DEVELOPMENT CO. B-14

Location-Galveston County, Texas

Township-Range-Section location- 5S-41E-4

API number- 42-167-

Log type- Induction Electrical

Logs available from 2000 feet to 13074 feet. 
Curve type(s) available-

Self Potential

Short Normal

Amplified Short Normal

Induction Conductivity

Induction Resistivity

139. LOGDEX unique identification number- 213

Operator/Company-PAN AMERICAN

Well name- R. B. PETERS 5

Location-Galveston County, Texas

Township-Range-Section location- 5S-41E-4

Abstract/Tract/Block-A- 17

API number- 42-167- 988

HEADER data base unique number- 11293

Date of completion- $3 / 27 / 59$

Well Classificattion code- D\&A

Log type- Induction Electrical

Logs available from 250 feet to 15052 feet.

Curve type(s) available-

Self Potential

Short Normal

Ampl ified Short Normal

Long Norma 1

Lateral

Induction Conductivity

Induction Resistivity

140. LOGDEX unique identification number- 214

Operator/Company- SUPERIOR

Well name- SUPERIOR OIL COMPANY FEE 12

Location-Galveston County, Texas 
Summary of LOGDEX Data Base

Township-Range-Section location- 5S-41E-5

Abstract/Tract/Block-A- 17

API number- 42-167- 30057

HEADER data base unique number- 11294

Date of completion-12/3/71

Well Classificattion code- D\&A

Log type- Induction Electrical

Logs available from 3250 feet to 14366 feet.

Curve type(s) available-

Self Potential

Short Normal

Ampl ified Short Normal

Induction Conductivity

Induction Resistivity

141. LOGDEX unique identification number- 215

Operator/Company- SUPERIOR

We 11 name- SUPERIOR OIL COMPANY FEE 14

Location-Galveston County, Texas

Township-Range-Section location- 5S-41E-5

API number- 42-167-

Log type- Induction Electrical

Logs available from 1550 feet to 9016 feet.

Curve type(s) available-

Self Potential

Short Normal

Amplified Short Normal

Induction Conductivity

Induction Resistivity 
142. LOGDEX unique identification number- 216

Operator/Company- PAN AMERICAN

We11 name- DOLLAR BAY FIG CORP. 1

Location-Galveston County, Texas

Township-Range-Section location- 5S-41E-6

Abstract/Tract/Block-A- 4

API number-42-167- 965

HEADER data base unique number- 11797

Date of completion- $6 / 24 / 54$

Well Classificattion code- D\&A

Log type- Electrical

Logs available from 2130 feet to 11984 feet.

Curve type(s) available-

Self Potential

Short Normal

Amp 1 ified Short Normal

Long Normal

Lateral

143. LOGDEX unique identification number- 217

Operator/Company- PAN AMERICAN

Well name- SOUTH GILLOCK COMM. M-1

Location-Galveston County, Texas

Township-Range-Section location- 5S-41E-8

API number- 42-167-

Log type-Electrical

Logs available from 1600 feet to 9602 feet.

Curve type(s) available-

Self Potential 


\author{
Short Normal \\ Ampl ified Short Normal \\ Long Normal \\ Lateral
}

144. LOGDEX unique identification number- 218

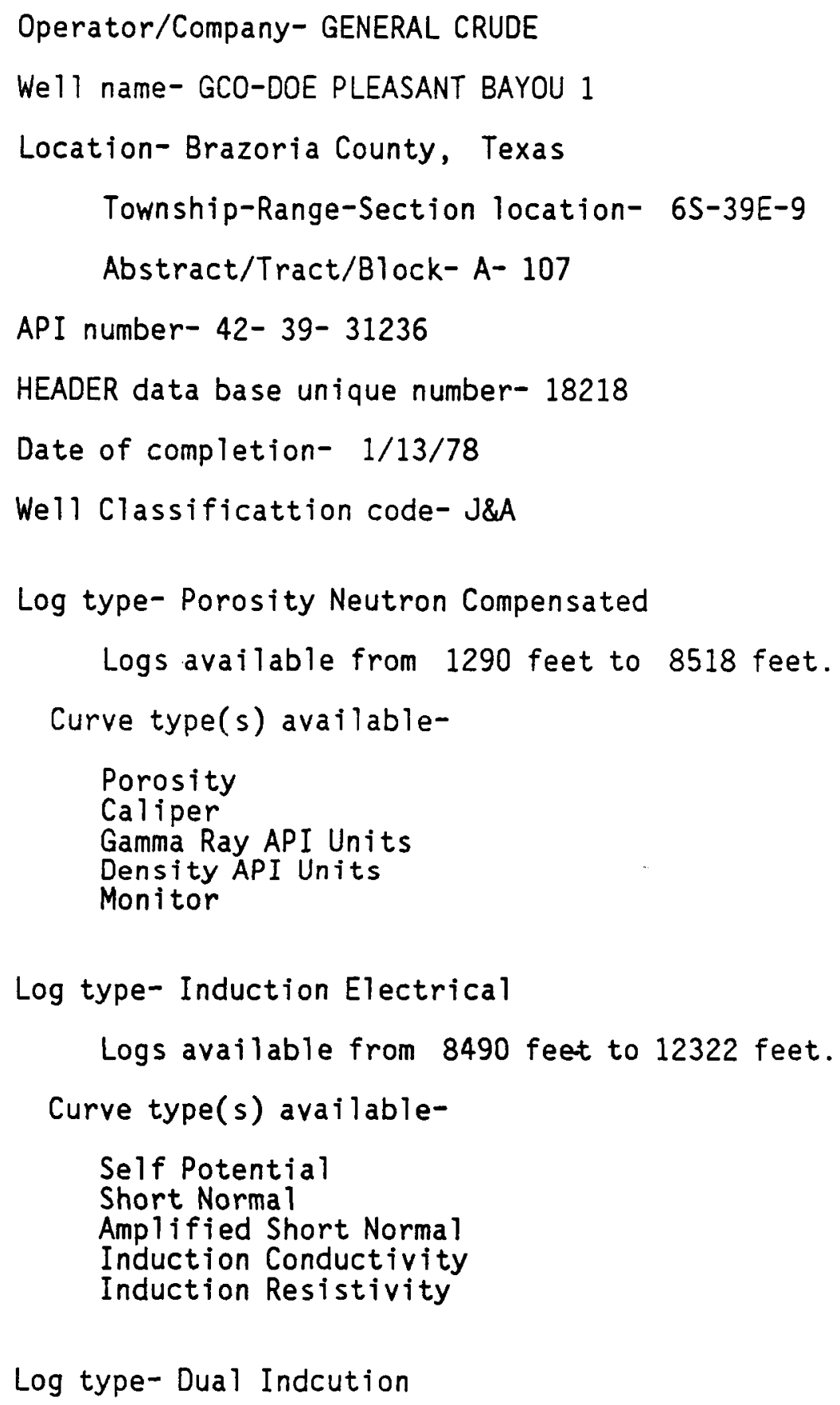

Log type- Dual Indcution 
Logs available from 1300 feet to 14772 feet.

Curve type(s) available-

Self Potential

Focussed Resistivity

Amplified Focussed Resistivity

Induction Conductivity

Induction Resistivity

Log type- Velocity

Logs available from 1300 feet to 14772 feet.

Curve type(s) available-

Self Potential

Acoustic Compensated

145. LOGDEX unique identification number- 219

Operator/Company- HOUSTON OIL \& MINERALS

Well name- E. W. BOYT 1

Location-Galveston County, Texas

Township-Range-Section location- 5S-42E-7

Abstract/Tract/Block-A- 162

API number- 42-167- 30127

HEADER data base unique number- 11800

Date of completion-12/29/73

Well Classificattion code- D\&A

Log type- Induction Electrical .

Logs available from 3500 feet to 13428 feet.

Curve type(s) available-

Self Potential

Induction Conductivity

Induction Resistivity

Focussed Resistivity

Amplified Focussed Resistivity 
Summary of LOGDEX Data Base

146. LOGDEX unique identification number- 220

Operator/Company- HOUSTON OIL \& MINERALS

Well name- 3421

Location-Galveston County, Texas

Township-Range-Section location- 5S-42E-7

Abstract/Tract/Block- T- 342

API number- 42-167- 30087

HEADER data base unique number- 20406

Date of completion- $9 / 25 / 72$

Well Classificattion code- GAS

Log type- Induction Electrical

Logs available from 200 feet to 12976 feet.

Curve type(s) available-

Self Potential

Induction Conductivity

Induction Resistivity

Focussed Resistivity

Amplified Focussed Resistivity

147. LOGDEX unique identification number- 222

Operator/Company- HUMBLE

Well name- $113 \mathrm{~A}-1$

Location-Galveston County, Texas

Township-Range-Section location- 5S-42E-9

API number- 42-167-

Log type- Electrical

Logs available from 260 feet to 14114 feet. 
Curve type(s) available-

Self Potential

Short Norma 1

Ampl ified Short Normal

Long Norma 1

Lateral

148. LOGDEX unique identification number- 223

Operator/Company- HUMBLE

We 11 name- C. M. ARMSTRONG 27

Location-Kenedy County, Texas

Township-Range-Section location-26S-18E-3

Abstract/Tract/Block-A- 3

API number- 42-261- 30073

HEADER data base unique number- 14963

Date of completion-12/18/72

Well Classificattion code- D\&A

Log type- Induction Electrical

Logs available from 2000 feet to 10736 feet.

Curve type(s) available-

Self Potential

Short Normal

Ampl ified Short Normal

Induction Resistivity

149. LOGDEX unique identification number- 224

Operator/Company- HUMBLE

We 11 name- CHARLES M. ARMSTRONG 8

Location-Kenedy County, Texas

Township-Range-Section location-25S-18E-8 
Summary of LOGDEX Data Base

\author{
Abstract/Tract/Block-A- 3 \\ API number- 42-261- 223 \\ HEADER data base unique number- 14868 \\ Date of completion- 6/28/55 \\ Well Classificattion code- D\&A \\ Log type- Electrical \\ Logs available from 100 feet to 16020 feet. \\ Curve type(s) available- \\ Self Potential \\ Short Normal \\ Long Norma 1
}

150. LOGDEX unique identification number- 225

Operator/Company- HUMBLE

We 11 name-S. K. EAST 54

Location- Kenedy County, Texas

Township-Range-Section location- 24S-18E-8

Abstract/Tract/Block-A- 36

API number- 42-261- 30067

HEADER data base unique number- 15170

Date of completion- $11 / 16 / 72$

Well Classificattion code- D\&A

Log type- Induction Electrical

Logs available from 2670 feet to 14470 feet.

Curve type(s) available-

Self Potential

Short Normal

Induction Resistivity 
151. LOGDEX unique identification number- 226

Operator/Company- HUMBLE

We 11 name-MRS. S. K. EAST 41

Location- Kenedy County, Texas

Township-Range-Section location-25S-18E-1

Abstract/Tract/Block-A- 36

API number- 42-261- 480

HEADER data base unique number- 11790

Date of completion- $8 / 5 / 68$

We1l Classificattion code- D\&A

Log type- Induction Electrical

Logs available from 2600 feet to 15066 feet.

Curve type(s) available-

Self Potential

Short Normal

Induction Resistivity

152. LOGDEX unique identification number- 227

Operator/Company- HUMBLE

We 11 name-MRS. S. K. EAST 22

Location- Kenedy County, Texas

Township-Range-Section location-25S-18E-3

Abstract/Tract/Block-A- 36

API number- 42-261- 393

HEADER data base unique number- 15105

Date of completion- 6/13/65

Well Classificattion code- D\&A 
Summary of LOGDEX Data Base

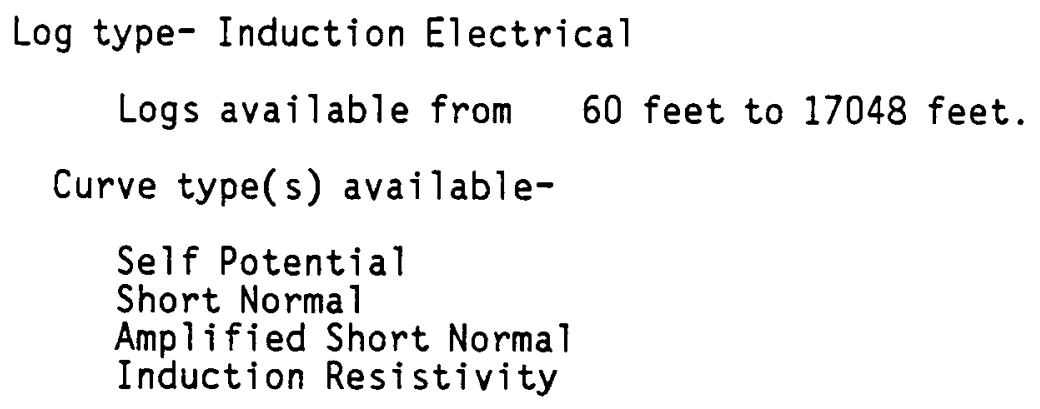

153. LOGDEX unique identification number- 228

Operator/Company- LAMAR HUNT

Well name- FLENIKEN 1

Location- Karnes County, Texas

Township-Range-Section location- 9S-19E-8

Abstract/Tract/Block-A- 13

API number- 42-255- 30121

HEADER data base unique number- 1197

Date of completion- $3 / 30 / 72$

Well Classificattion code- D\&A

Log type- Induction Electrical

Logs available from 4300 feet to 16042 feet.

Curve type(s) available-

Self Potential

Short Normal

Induction Resistivity

154. LOGDEX unique identification number- 229

Operator/Company- DIXEL RESOURCES

Wel1 name- R. J. ROEDER 1

Location- DeWitt County, Texas

Page 102 
Township-Range-Section location- 9S-20E-3

Abstract/Tract/Block- A- 223

API number- 42-123- 30170

HEADER data base unique number- 11407

Date of completion- 8/5/74

Well Classificattion code- D\&A

Log type- Induction Electrical

Logs available from 3500 feet to 13666 feet.

$\cdots$ Curve type(s) available-

Self Potential

Induction Resistivity

Focussed Resistivity

155. LOGDEX unique identification number- 230

Operator/Company- LONE STAR PRODUCING

We 11 name- ALBERT JANK 1

Location-Goliad County, Texas

Township-Range-Section location-10S-20E-6

Abstract/Tract/Block- A- 340

API number- 42-175- 2112

HEADER data base unique number- 2006

Date of completion- 2/4/69

Well Classificattion code- D\&A .

Log type- Induction Electrical

Logs available from 1800 feet to 14020 feet.

Curve type(s) available-

Self Potential

Short Normal

Induction Resistivity 
Summary of LOGDEX Data Base

156. LOGDEX unique identification number- 232

Operator/Company- EXXON

We 11 name- C. M. ARMSTRONG 31

Location-Kenedy County, Texas

Township-Range-Section location-26S-18E-2

Abstract/Tract/Block-A- 3

API number- 42-261- 30119

HEADER data base unique number- 15102

Date of completion-11/18/74

Well Classificattion code- GAS

Log type- Induction Electrical

Logs available from 2050 feet to 10230 feet.

Curve type(s) available-

Self Potential

Short Normal

Induction Resistivity

157. LOGDEX unique identification number- 233

Operator/Company-SHELL

Well name- E. A. SIBLEY 1

Location- Lavaca County, Texas

Township-Range-Section location- 7S-25E-5

Abstract/Tract/Block- A- 554

API number- 42-285- 473

HEADER data base unique number- 11337

Date of completion-11/12/62

Well Classificattion code- D\&A 
Log type- Induction Electrical

Logs available from 3100 feet to 15600 feet.

Curve type(s) available-

Self Potential

Short Normal

Induction Resistivity

158. LOGDEX unique identification number- 234

Operator/Company- GULF

Well name-W. V. ROELL 1

Location-Victoria County, Texas

Township-Range-Section location- 9S-24E-4

Abstract/Tract/Block-A- 20

API number- 42-469- 774

HEADER data base unique number- 15072

Date of completion-11/28/59

We1l Classificattion code- D\&A

Log type- Induction Electrical

Logs available from 600 feet to 10046 feet.

Curve type(s) available-

Self Potential

Short Normal

Induction Resistivity

159. LOGDEX unique identification number- 235

Operator/Company- CHEVRON

We 11 name- R. G. JACOBS 1

Location-Goliad County, Texas

Township-Range-Section location- 10S-22E-9 
Summary of LOGDEX Data Base

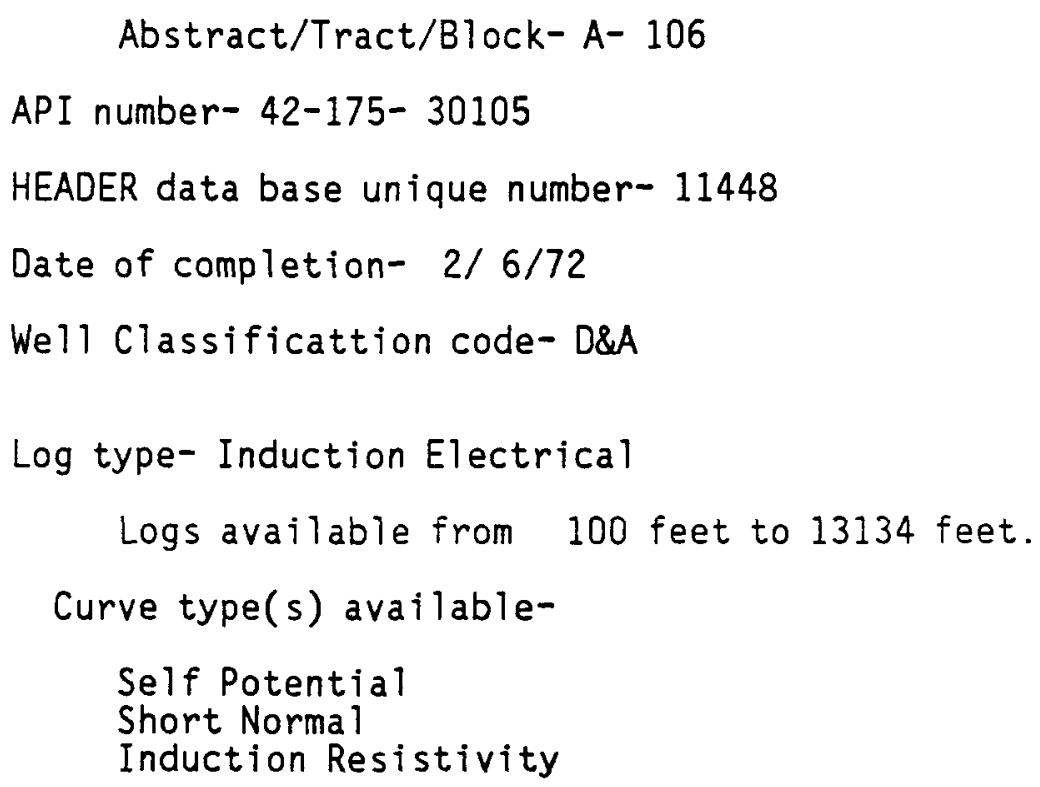

160. LOGDEX unique identification number- 236

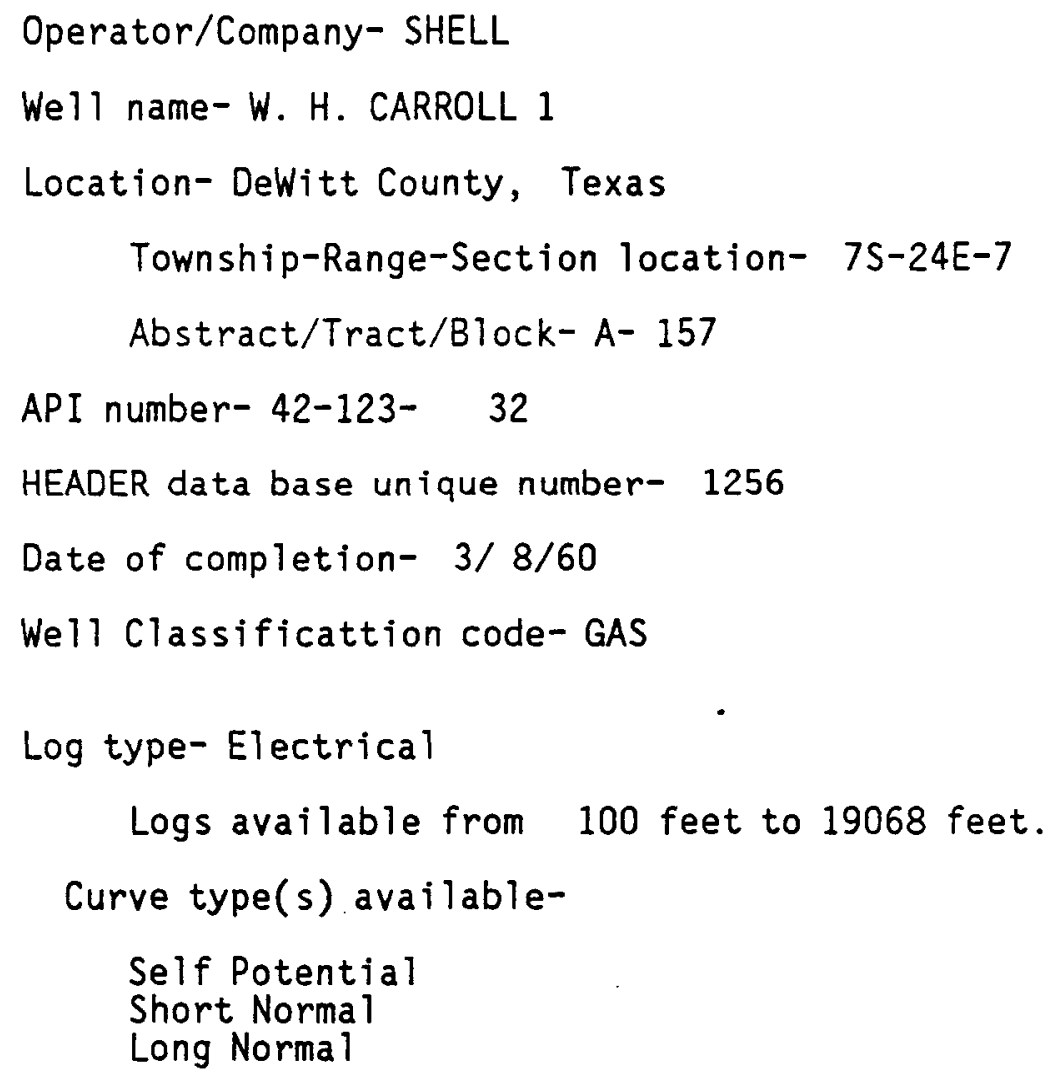


161. LOGDEX unique identification number- 237

Operator/Company-MAGNOLIA

We 11 name- F. G. MANN 1

Location-DeWitt County, Texas

Township-Range-Section location- 8S-20E-6

Abstract/Tract/Block-A- 451

API number- 42-123- 981

HEADER data base unique number- 1261

Date of completion- $8 / 20 / 53$

We 11 Classificattion code- D\&A

Log type- Electrical

Logs available from 100 feet to 10376 feet.

Curve type(s) available-

Self Potential

Short Normal

Long Norma 1

162. LOGDEX unique identification number- 238

Operator/Company-WEAVER ACQUISITION

We 11 name- HILDRED BOLTON 1

Location-DeWitt County, Texas

Township-Range-Section location- 7S-23E-7

Abstract/Tract/Block- A- 421

API number- 42-123- 30347

HEADER data base unique number- 15079

Date of completion- 1/16/77

Well Classificattion code- D\&A 
Summary of LOGDEX Data Base

Log type- Induction Electrical

Logs available from 2000 feet to 11212 feet.

Curve type(s) available-

$$
\begin{aligned}
& \text { Self Potential } \\
& \text { Short Normal } \\
& \text { Induction Resistivity }
\end{aligned}
$$

163. LOGDEX unique identification number- 239

Operator/Company- MONSANTO

We 11 name- KULAWIK 1

Location- DeWitt County, Texas

Township-Range-Section location- 9S-21E-5

Abstract/Tract/Block-A- 116

API number- 42-123- 1083

HEADER data base unique number- 20008

Date of completion- 6/26/68

Wel1 Classificattion code- GAS

Log type- Induction Electrical

Logs available from 1600 feet to 13034 feet.

Curve type(s) available-

Self Potential

Short Norma 1

Induction Resistivity

164. LOGDEX unique identification number- 240

Operator/Company- SHELL

Well name- CORA S. BROWN 1

Location- DeWitt County, Texas

Township-Range-Section location- 8S-21E-4 
Abstract/Tract/Block-A- 219

API number-42-123- 30020

HEADER data base unique number- 1258

Date of completion- $4 / 16 / 70$

We 11 Classificattion code- D\&A

Log type- Induction Electrical

Logs available from 100 feet to 15700 feet.

Curve type(s) available-

Self Potential

Short Normal

Induction Resistivity

165. LOGDEX unique identification number- 241

Operator/Company- LONE STAR PRODUCING

We 11 name- BERTHA MUELLER 1

Location- DeWitt County, Texas

Township-Range-Section location- 8S-22E-7

API number- 42-123-

Log type- Induction Electrical

Logs available from 1750 feet to 13524 feet.

Curve type(s) available-

Self Potential

Short Normal

Induction Resistivity

166. LOGDEX unique identification number- 242

Operator/Company- ATLANTIC RICHFIELD

Well name- R. A. HARTMAN 1 
Summary of LOGDEX Data Base

\author{
Location- DeWitt County, Texas \\ Township-Range-Section location- $8 S-23 E-8$ \\ API number- 42-123-
}

Log type- Induction Electrical

Logs available from 2100 feet to 10396 feet.

Curve type(s) available-

$$
\begin{aligned}
& \text { Self Potential } \\
& \text { Induction Resistivity } \\
& \text { Focussed Resistivity }
\end{aligned}
$$

Log type- Velocity

Logs available from 2020 feet to 16002 feet.

Curve type(s) available-

Self Potential

Acoustic Compensated

Caliper

Gamma Ray API Units

167. LOGDEX unique identification number- 243

Operator/Company- STANDARD OIL COMPANY OF TEXAS

We 11 name- LOUIS TRAUTWEIN ET AL 1

Location- DeWitt County, Texas

Township-Range-Section location- 9S-22E-2

Abstract/Tract/Block-A- 172

API number- 42-123- 557

HEADER data base unique number- 12370

Date of completion- $5 / 20 / 61$

Well Classificattion code- GAS

Log type- Induction Electrical

Logs available from 1850 feet to 13752 feet. 
Curve type(s) available-

Self Potential

Short Normal

Induction Resistivity

Log type- Velocity

Logs available from 100 feet to 13738 feet.

Curve type(s) available-

Self Potential

Acoustic Compensated

168. LOGDEX unique identification number- 244

Operator/Company- LONE STAR PRODUCING

We 11 name- FRIEDA VOGT 1

Location-Victoria County, Texas

Township-Range-Section location- 9S-23E-5

API number- 42-469-

Log type- Induction Electrical

Logs available from 1800 feet to 12106 feet.

Curve type(s) available-

Self Potential

Short Normal

Induction Resistivity

169. LOGDEX unique identification number- 245

Operator/Company-MOBIL .

Wel1 name-M. L. SPEARY 1

Location- Karnes County, Texas

Township-Range-Section location- 10S-19E-2 
Summary of LOGDEX Data Base

\author{
Abstract/Tract/B1ock-A- 290 \\ API number- 42-255- 270 \\ HEADER data base unique number- 12480 \\ Date of completion- $3 / 29 / 61$ \\ We11 Classificattion code- GAS \\ Log type- Induction Electrical \\ Logs available from 1100 feet to 14402 feet. \\ Curve type(s) available- \\ Self Potential \\ Short Normal \\ Induction Resistivity
}

170. LOGDEX unique identification number- 246

Operator/Company - HUMBLE OIL AND REFINING

Well name- MARY A. MEYER 1

Location-Goliad County, Texas

Township-Range-Section location- 10S-21E-2

API number- 42-175-

Log type- Induction Electrical

Logs available from 1600 feet to 13042 feet.

Curve type(s) available-

Self Potential

Short Normal

Induction Resistivity

171. LOGDEX unique identification number- 247

Operator/Company- PAN AMERICAN

We 11 name- C. A. O'CONNOR 1

Page 112 


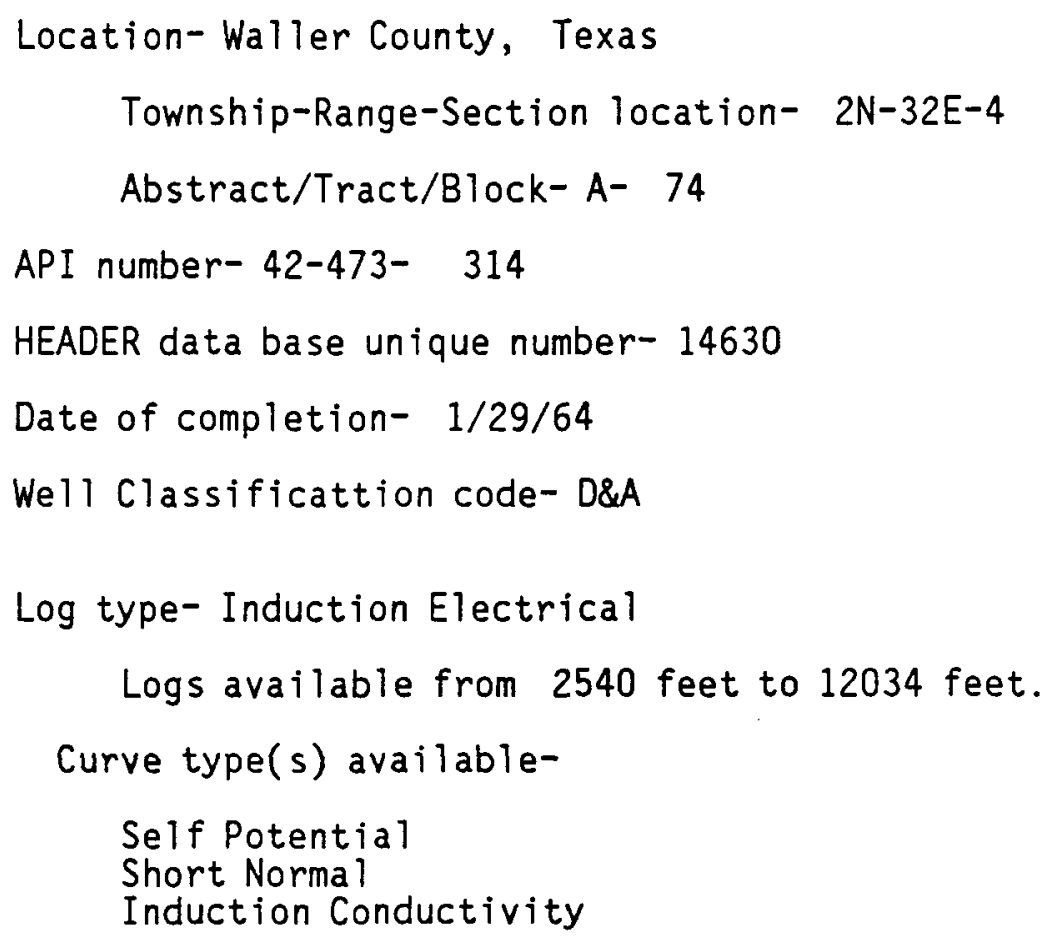

172. LOGDEX unique identification number- 248

Operator/Company- SINCLAIR OIL AND GAS

We 11 name- FOSTER LUMBER CO. 1

Location-Montgomery County, Texas

Township-Range-Section location- 2N-39E-4

API number- 42-339-

Log type- Electrical

Logs available from 2600 feet to 12002 feet.

Curve type(s) available-

Self Potential

Short Normal

Long Normal

173. LOGDEX unique identification number- 249 
Summary of LOGDEX Data Base

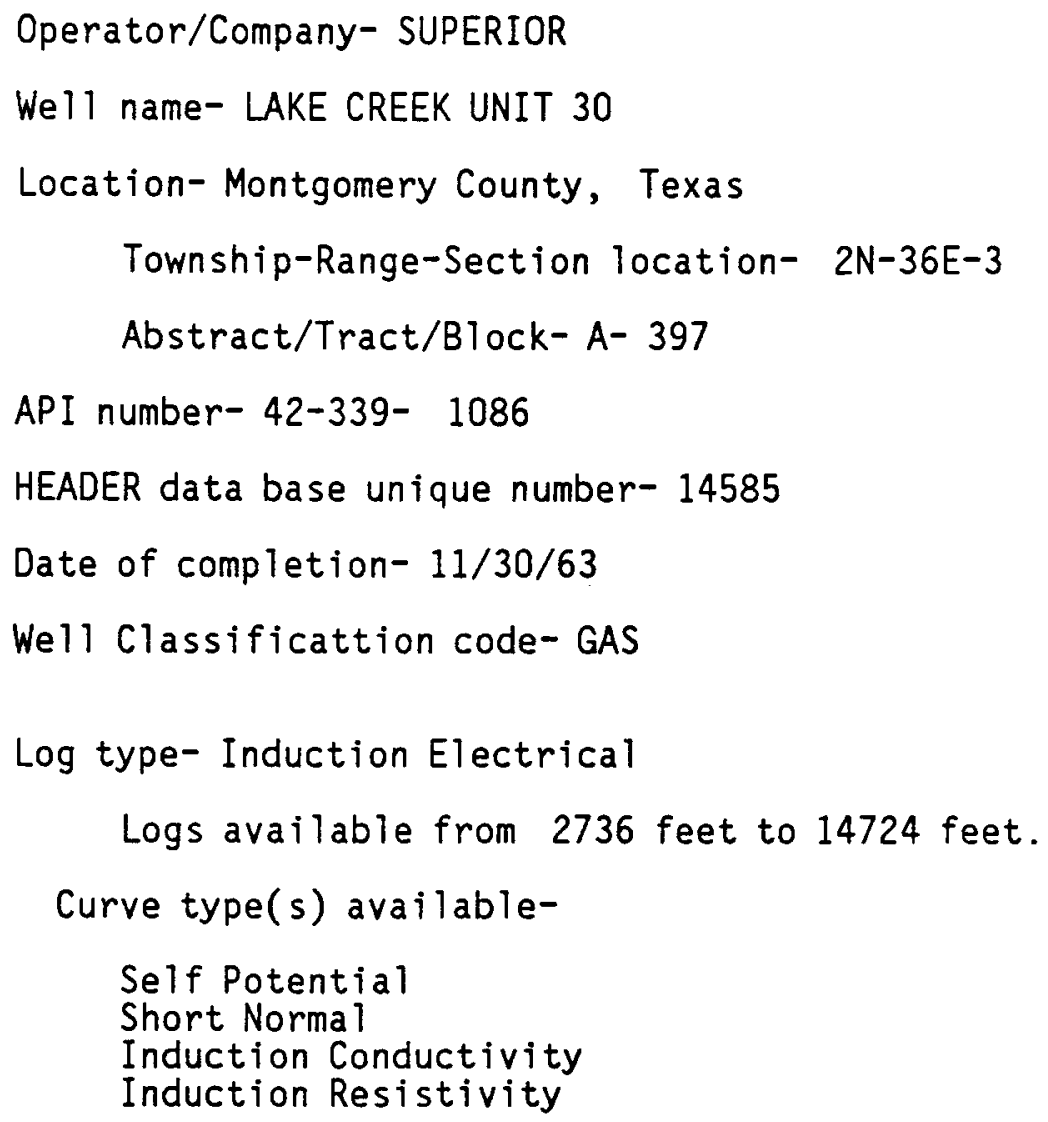

174. LOGDEX unique identification number- 250

Operator/Company-MOBIL

Well name- BENDER ESTATE FARM 1

Location-Montgomery County, Texas

Township-Range-Section location- $1 \mathrm{~N}-38 \mathrm{E}-3$

Abstract/Tract/Block-A- 351

API number- 42-339- 1739

HEADER data base unique number- 14587

Date of completion- 8/6/62

Well Classificattion code- D\&A

Log type- Induction Electrical

Logs available from 100 feet to 14884 feet. 
Curve type(s) available-

Self Potential

Short Normal

Induction Conductivity

175. LOGDEX unique identification number- 252

Operator/Company-SINCLAIR OIL AND GAS

We 11 name- HENRY KREZDORN 1

Location-Harris County, Texas

Township-Range-Section location- 1N-33E-1

API number- 42-201-

Log type- Electrical

Logs available from 2500 feet to 12012 feet.

Curve type(s) available-

Self Potential

Short Normal

Long Norma 1

176. LOGDEX unique identification number- 253

Operator/Company- SUN

We 11 name- FOSTER 34

Location-Montgomery County, Texas

Township-Range-Section location- 2N-38E-3

Abstract/Tract/Block-A- 98

API number- 42-339- 30386

HEADER data base unique number- 11707

Date of completion- 1/23/77

Well Classificattion code- GAS 
Log type- Induction Electrical

Logs available from 2000 feet to 11486 feet.

Curve type(s) available-

Self Potential

Induction Conductivity

Focussed Resistivity

177. LOGDEX unique identification number- 254

Operator/Company- SUPERIOR

Well name- T. E. MOSTYN 1

Location-Montgomery County, Texas

Township-Range-Section location- 2N-35E-2

API number- 42-339-

Log type- Induction Electrical

Logs available from 1800 feet to 11492 feet.

Curve type(s) available-

Self Potential

Short Norma 1

Induction Conductivity

178. LOGDEX unique identification number- 255

Operator/Company- CARELTON SPEED, JR.

We 11 name- SAUERBRUNN ET AL 1

Location-Grimes County, Texas

Township-Range-Section location- 2N-34E-3

API number- 42-185-

Log type- Induction Electrical 
Logs available from 1400 feet to 12024 feet.

Curve type(s) available-

Self Potential

Short Norma 1

Induction Conductivity

Induction Resistivity

179. LOGDEX unique identification number- 256

Operator/Company- MANA OIL \& ASSOCIATED OIL AND GAS

Well name- J. H. SNITH 1

Location-Waller County, Texas

Township-Range-Section location- $2 \mathrm{~N}-33 \mathrm{E}-4$

API number- 42-473-

Log type- Induction Electrical

Logs available from 1550 feet to 11042 feet.

Curve type(s) available-

Self Potential

Short Normal

Induction Conductivity

180. LOGDEX unique identification number- 257

Operator/Company- HUMBLE

We 11 name- FOSTER LUMBER COMPANY 1

Location- Harris County, Texas

Township-Range-Section location- $1 \mathrm{~N}-39 \mathrm{E}-7$

Abstract/Tract/Block- A- 757

API number- 42-201- 1032

HEADER data base unique number- 11096

Date of completion- $12 / 29 / 62$ 
Summary of LOGDEX Data Base

\author{
Well Classificattion code- D\&A \\ Log type- Induction Electrical \\ Logs available from 150 feet to 13216 feet. \\ Curve type(s) available- \\ Self Potential \\ Short Norma 1 \\ Induction Conductivity
}

181. LOGDEX unique identification number- 258

Operator/Company- HUMBLE

Wel1 name- TOMBALL GAS UNIT 111

Location- Harris County, Texas

Township-Range-Section location- 1N-36E-4

API number- 42-201-

Log type- Induction Electrical

Logs available from 200 feet to 10212 feet.

Curve type(s) available-

Self Potential

Short Normal

Induction Conductivity

Gamma Ray API Units

182. LOGDEX unique identification number- 259

Operator/Company- SINCLAIR

We 11 name- MCCRABB 1

Location- Montgomery County, Texas

Township-Range-Section location- $1 \mathrm{~N}-34 \mathrm{E}-1$

Abstract/Tract/Block-A- 234

Page 118 
API number- 42-339- 1015

HEADER data base unique number- 14877

Date of completion- 3/24/55

Well Classificattion code- D\&A

Log type- Electrical

Logs available from 2540 feet to 11022 feet.

Curve type(s) available-

Self Potential

Short Normal

Long Normal

183. LOGDEX unique identification number- 261

Operator/Company- GOODALE BERTMAN

Well name- E. D. MAYNARD 1

Location- Montgomery County, Texas

Township-Range-Section location- $2 \mathrm{~N}-38 \mathrm{E}-2$

API number- 42-339-

Log type- Induction Electrical

Logs available from 2030 feet to 10612 feet.

Curve type(s) available-

Self Potential

Short Normal

Induction Conductivity

Induction Resistivity

184. LOGDEX unique identification number- 262

Operator/Company- GENERAL CRUDE

We 11 name- GCO-DOE PLEASANT BAYOU 2 


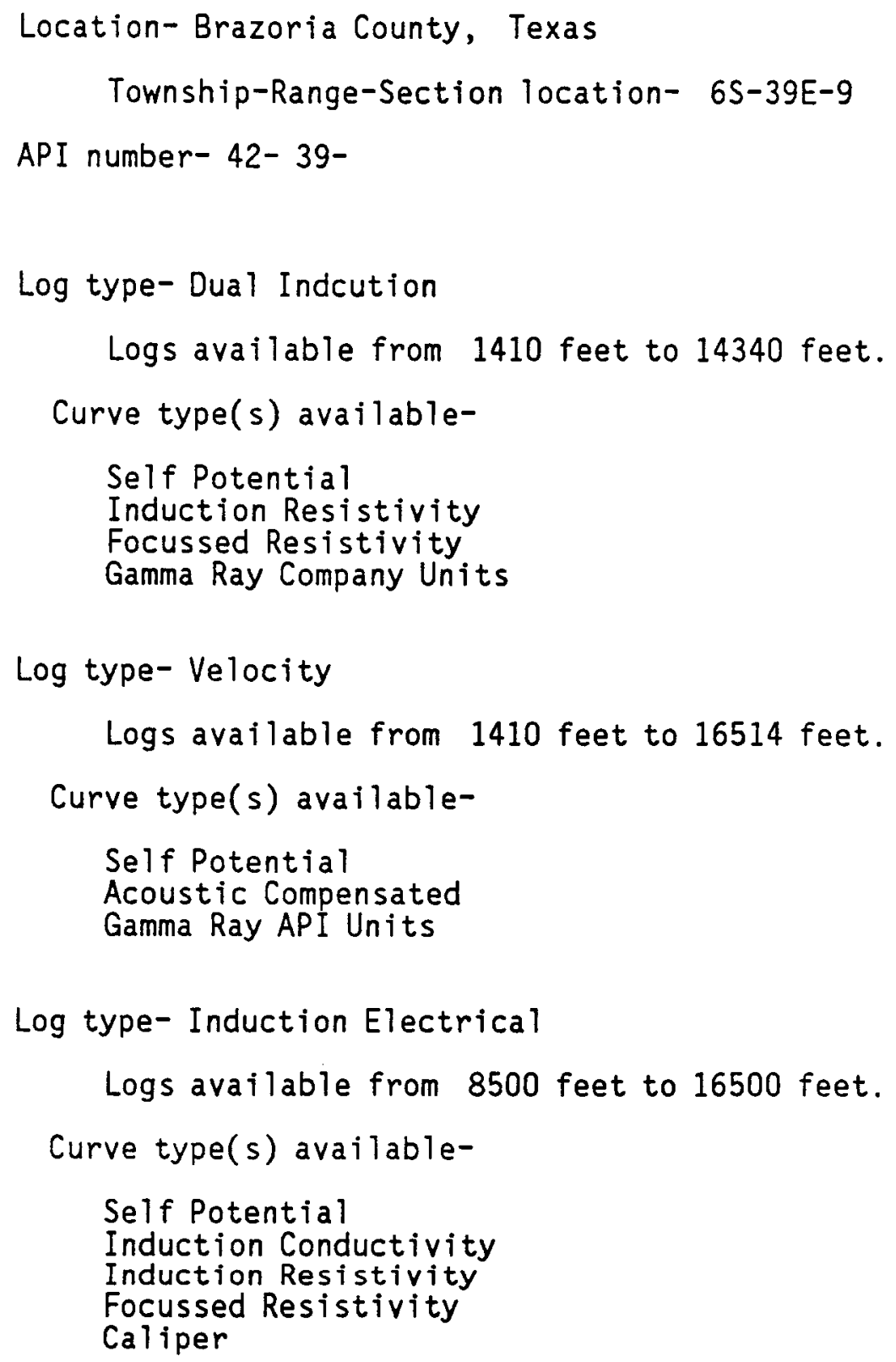

Log type- Temperature

Logs available from 160 feet to 16458 feet. Curve type(s) available-

Temperature

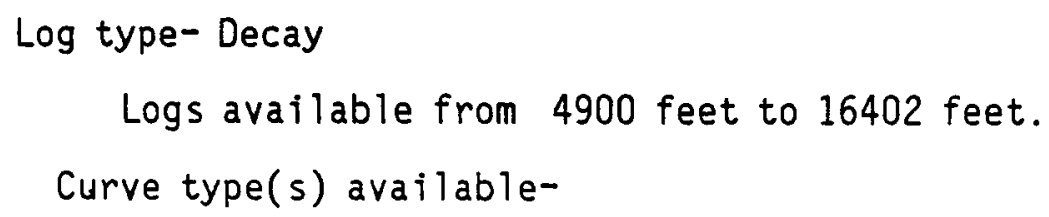


Sigma

Ratio

Monitor

Log type- Porosity Neutron Compensated

Logs available from 14230 feet to 16522 feet.

Curve type(s) available-

Porosity

Caliper

Gamma Ray API Units

Density API Units

Monitor

Log type- Spectralog

Logs available from 13700 feet to 16426 feet.

Curve type(s) available-

Uranium

Thorium

Potassium

Gamma Ray API Units

185. LOGDEX unique identification number- 263

Operator/Company- SPARTA OIL

Wel1 name- J. HARVEY SHUTTLES ET AL 1

Location- Harris County, Texas

Township-Range-Section location- 2S-37E-8

Abstract/Tract/Block-A- 31

API number- 42-201- 646

HEADER data base unique number- 11070

Date of completion- $9 / 12 / 50$

Well Classificattion code- D\&A

Log type- Induction Electrical

Logs available from 100 feet to 8860 feet. 
Summary of LOGDEX Data Base

\author{
Curve type(s) available- \\ Self Potential \\ Short Normal \\ Induction Conductivity
}

186. LOGDEX unique identification number- 264

Operator/Company- CROWN CENTRAL, ACORN AND CAULKINS

We 11 name- LUCILLE FISHER 1

Location- Harris County, Texas

Township-Range-Section location- 2S-35E-8

Abstract/Tract/Block- A- 193

API number- 42-201- 4130

HEADER data base unique number- 11190

Date of completion- 5/4/60

Well Classificattion code- D\&A

Log type- Induction Electrical

Logs available from 2080 feet to 8180 feet.

Curve type(s) available-

Self Potential

Short Norma ]

Induction Conductivity

187. LOGDEX unique identification number- 265

Operator/Company- EXXON

Wel1 name- KGFU WELL \#W-35 1

Location-Waller County, Texas

Township-Range-Section location- 2S-34E-9

API number- 42-473- 
Log type- Induction Electrical

Logs available from 2470 feet to 11620 feet.

Curve type(s) available-

Self Potential

Induction Resistivity

Focussed Resistivity

188. LOGDEX unique identification number- 266

Operator/Company- EXXON

We 11 name- KGFU WELL \#W-45 2

Location-Waller County, Texas

Township-Range-Section location- 2S-33E-1

Abstract/Tract/Block-A- 52

API number- 42-473- 125

HEADER data base unique number- 11174

Date of completion- $8 / 30 / 58$

We 11 Classificattion code- D\&A

Log type- Induction Electrical

Logs available from 2950 feet to 11242 feet.

Curve type(s) available-

Self Potential

Induction Conductivity

Induction Resistivity

Focussed Resistivity

189. LOGDEX unique identification number- 267

Operator/Company-MOUND

Well name- L. F. FUQUA 1

Location-Waller County, Texas 
Summary of LOGDEX Data Base

\author{
Township-Range-Section location- 2S-32E-1 \\ API number- 42-473- \\ Log type- Electrical \\ Logs available from 980 feet to 8178 feet. \\ Curve type(s) available- \\ Self Potential \\ Short Norma 1 \\ Long Norma ?
}

190. LOGDEX unique identification number- 268

Operator/Company- SOUTHERN NATURAL GAS

Wel1 name- FRANK UHYREK 1

Location-Austin County, Texas

Township-Range-Section location- 3S-32E-9

Abstract/Tract/Block-A- 164

API number- 42- 15- 683

HEADER data base unique number- 11585

Date of completion- 12/15/66

Well Classificattion code- D\&A

Log type- Induction Electrical

Logs available from 200 feet to 13026 feet.

Curve type(s) available-

Self Potential

Short Norma 1

Induction Conductivity

Induction Resistivity

191. LOGDEX unique identification number- 269

Page 124 


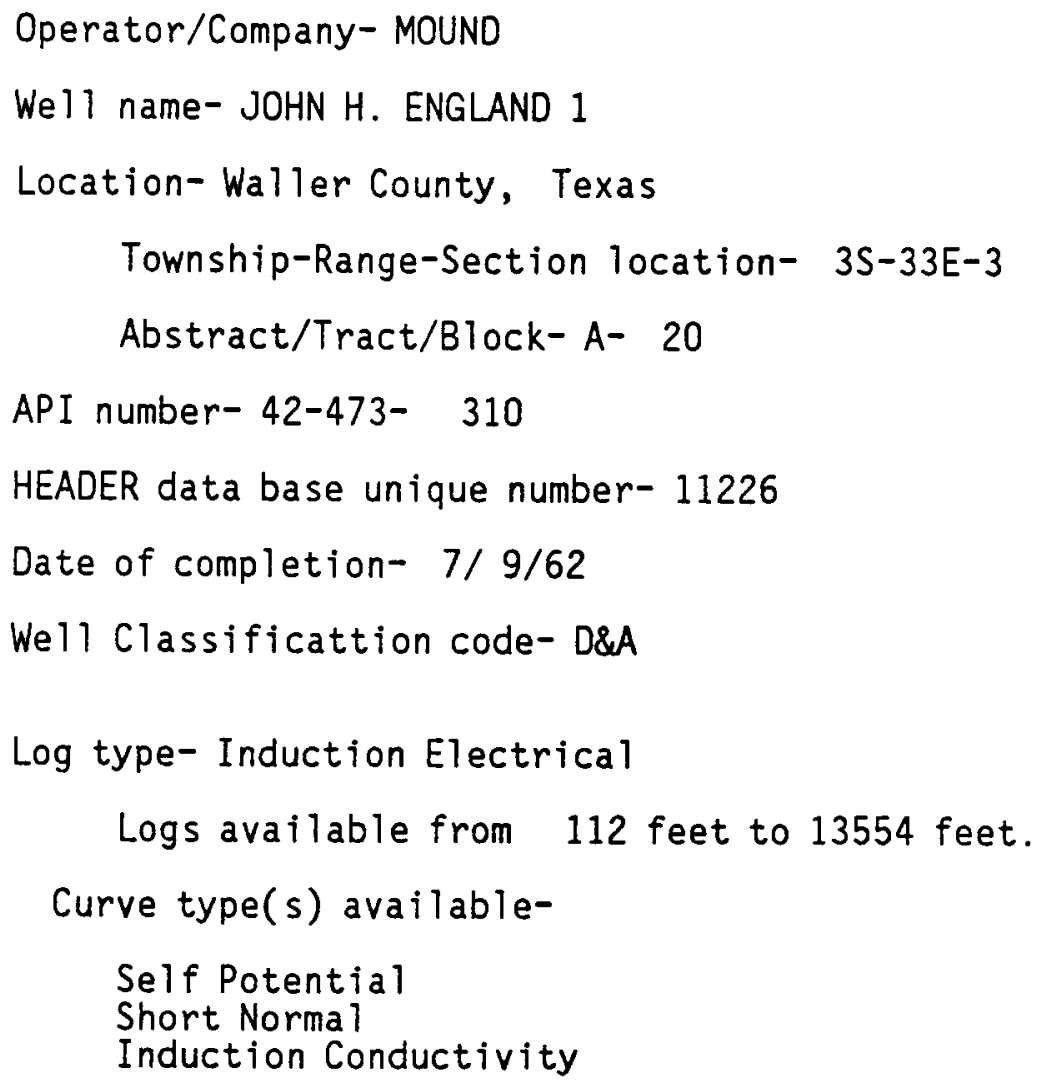

192. LOGDEX unique identification number- 270

Operator/Company- J P OWEN

We 11 name- JOHN H. ENGLAND 1

Location-Waller County, Texas

Township-Range-Section location- 3S-33E-3

Abstract/Tract/Block-A- 20

API number- 42-473- 310

HEADER data base unique number- 11587

Date of completion- 7/9/64

Well Classificattion code- D\&A

Log type- Induction Electrical

Logs available from 12050 feet to 16070 feet. 


\section{Curve type(s) available- \\ Self Potential \\ Short Normal \\ Induction Conductivity}

193. LOGDEX unique identification number- 271

Operator/Company- PURE

We 11 name- N. A. BINZ I

Location- Fort Bend County, Texas

Township-Range-Section location- 3S-34E-5

API number- 42-157-

Log type- Induction Electrical

Logs available from 280 feet to 13538 feet.

Curve type(s) available-

Self Potential

Short Normal

Lateral

Induction Conductivity

194. LOGDEX unique identification number- 272

Operator/Company- CERRO DE PASCO ET AL

We 11 name- EDGAR BROWN ET AL 1

Location- Harris County, Texas .

Township-Range-Section location- 3S-35E-1

Abstract/Tract/B1ock-A- 19

API number- 42-201- 3113

HEADER data base unique number- 11598

Date of completion- $8 / 23 / 61$

We11 Classificattion code- D\&A 
Log type- Induction Electrical

Logs available from 1024 feet to 8362 feet.

Curve type(s) available-

Self Potential

Short Norma 1

Induction Conductivity

195. LOGDEX unique identification number- 273

Operator/Company- CERRO DE PASCO \& CH OSMOND

Well name- BESSIE BUDEWIG 1

Location-Harris County, Texas

Township-Range-Section location- 3S-36E-4

Abstract/Tract/Block- A- 406

API number- 42-201- 4426

HEADER data base unique number- 11229

Date of completion-12/7/56

Well Classificattion code- D\&A

Log type- Electrical

Logs available from 1266 feet to 9022 feet.

Curve type(s) available-

Self Potential

Short Normal

Long Normal

196. LOGDEX unique identification number- 274

Operator/Company- EDWIN L COX

Well name- J. W. ATKINSON 1

Location-McMullen County, Texas

Township-Range-Section location- 16S-12E-1 
Summary of LOGDEX Data Base

Abstract/Tract/Block-A- 657

API number- 42-311- 30504

HEADER data base unique number- 12993

Date of completion- 1/12/77

Well Classificattion code- D\&A

Log type- Induction Electrica 1

Logs available from 830 feet to 9552 feet.

Curve type(s) available-

Self Potential

Lateral

Induction Conductivity

197. LOGDEX unique identification number- 275

Operator/Company- MID CENTURY OIL AND GAS

We 11 name- WILLIAM HUBBEND 1

Location- McMullen County, Texas

Township-Range-Section location-16S-11E-1

Abstract/Tract/Block-A-1043

API number- 42-311- 1843

HEADER data base unique number- 1211

Date of completion- $8 / 15 / 66$

Well Classificattion code- D\&A

Log type- Induction Electrical

Logs available from 1010 feet to 8058 feet.

Curve type(s) available-

Self Potential

Short Norma 1

Induction Conductivity 
198. LOGDEX unique identification number- 276

Operator/Company- HARKINS AND COMPANY AND HUMBLE

We 11 name- VIRGINIA RAGSDALE SEC 1201

Location- Duval County, Texas

Township-Range-Section location-16S-13E-9

API number- 42-131-

Log type- Induction Electrical

Logs available from 1024 feet to 11172 feet.

Curve type(s) available-

Self Potential

Short Norma 1

Induction Conductivity

199. LOGDEX unique identification number- 277

Operator/Company- MARINE CONTRACTORS AND SUPPLY

Well name- HAHL - WEIDERNEHR 1

Location- Duva1 County, Texas

Township-Range-Section location-17S-11E-7

Abstract/Tract/Block- A- 435

API number- 42-131- 31154

HEADER data base unique number- 13102

Date of completion- 3/12/75

Well Classificattion code- GAS

Log type- Induction Electrical

Logs available from 580 feet to 10230 feet.

Curve type(s) available- 
Self Potential

Short Normal

Induction Conductivity

200. LOGDEX unique identification number- 278

Operator/Company- TEXACO

Well name- S. G. MARSHALL - STATE 1

Location- Duval County, Texas

Township-Range-Section location-17S-12E-1

Abstract/Tract/Block- A-2123

API number- 42-131- 2067

HEADER data base unique number- 11639

Date of completion- $4 / 10 / 63$

We 11 Classificattion code- GAS

Log type- Induction Electrical

Logs available from 100 feet to 13522 feet.

Curve type(s) available-

Self Potential

Short Norma 1

Induction Conductivity

201. LOGDEX unique identification number- 279

Operator/Company- EL PASO NATURAL.GAS

Well name- FARMERS LIFE YATES RANCH 1

Location- Duval County, Texas

Township-Range-Section location- 17S-13E-6

API number- 42-131-

Log type- Induction Electrical 
Logs available from 1500 feet to 10306 feet.

Curve type(s) available-

Self Potential

Short Normal

Induction Conductivity

202. LOGDEX unique identification number- 280

Operator/Company- HUMBLE

Wel1 name- PAUL E. WHITE "B" 98

Location- Duval County, Texas

Township-Range-Section location- 18S-11E-2

Abstract/Tract/Block-A- 808

API number- 42-131- 10868

HEADER data base unique number- 11659

Date of completion- $7 / 12 / 66$

Well Classificattion code- D\&A

Log type- Induction Electrical

Logs available from 2620 feet to 11176 feet.

Curve type(s) available-

Self Potential

Short Normal

Induction Conductivity

Gamma Ray API Units

203. LOGDEX unique identification number- 281

Operator/Company- MOODY PROPERTIES AND SOUTHLAND DRILLING

Well name- NICOLAS CANTU 1

Location- Duval County, Texas

Township-Range-Section location- 18S-13E-4 
Summary of LOGDEX Data Base

API number- 42-131-

Log type- Induction Electrical

Logs available from 262 feet to 7512 feet.

Curve type(s) available-

Self Potential

Short Normal

Induction Conductivity

204. LOGDEX unique identification number- 282

Operator/Company- EASON

We 11 name- PETERS ESTATE 1

Location- Duval County, Texas

Township-Range-Section location- 19S-11E-9

Abstract/Tract/Block- A- 385

API number- 42-131- 31372

HEADER data base unique number- 13316

Date of completion- 3/17/75

Well Classificattion code- GAS

Log type- Induction Electrical

Logs available from 1200 feet to 11270 feet.

Curve type(s) available-

Self Potential

Induction Conductivity

Focussed Resistivity

205. LOGDEX unique identification number- 283

Operator/Company-SHELL

We 11 name- J. B. STEGALL "A" 1

Page 132 


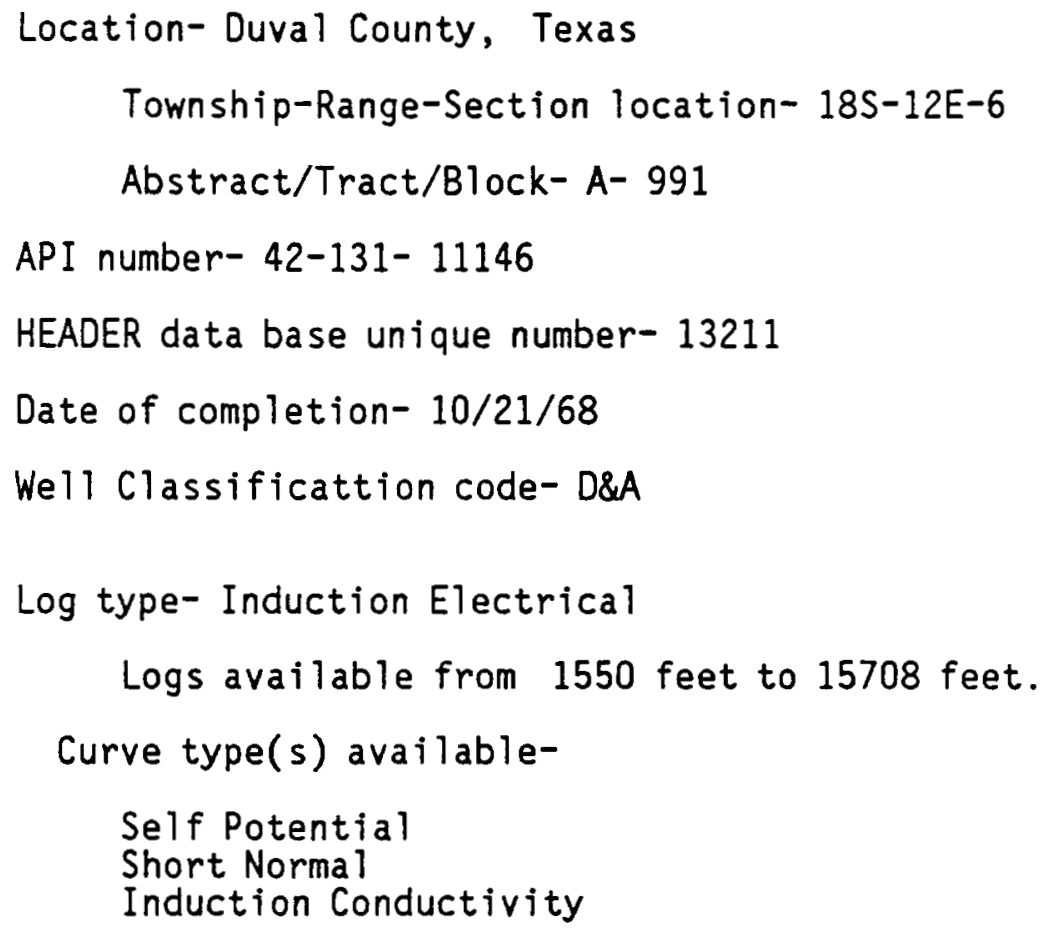

206. LOGDEX unique identification number- 284

Operator/Company- CHERRYVILLE CORPORATION

We 11 name-WILLIAMS 1

Location-Live Oak County, Texas

Township-Range-Section location-14S-16E-1

Abstract/Tract/Block-A- 178

API number- 42-297- 2782

HEADER data base unique number- 12869

Date of completion- 4/20/68

Well Classificattion code- D\&A

Log type- Induction Electrical

Logs available from 1550 feet to 10428 feet.

Curve type(s) available-

Self Potential

Short Normal 
Summary of LOGDEX Data Base

\section{Induction Conductivity}

207. LOGDEX unique identification number- 285

Operator/Company- HUMBLE

Well name- C. L. MCCASLIN 12

Location- Live Oak County, Texas

Township-Range-Section location-16S-16E-3

Abstract/Tract/Block-A- 86

API number- 42-297-30301

HEADER data base unique number- 20212

Date of completion- $12 / 14 / 71$

Well Classificattion code- GAS

Log type- Induction Electrical

Logs available from 1640 feet to 7032 feet.

Curve type(s) available-

Self Potential

Short Normal

Induction Conductivity

208. LOGDEX unique identification number- 286

Operator/Company- KILROY , TESORO AND SUBURBAN

Wel1 name- ROYCE PASSMORE 1

Location- Live Oak County, Texas

Township-Range-Section location- 16S-15E-3

Abstract/Tract/Block-A- 102

API number- 42-297- 30331

HEADER data base unique number- 15803

Date of completion- $6 / 25 / 72$ 
Well Classificattion code- GAS

Log type- Induction Electrical

Logs available from 2520 feet to 11590 feet.

Curve type(s) available-

Self Potential

Induction Conductivity

Focussed Resistivity

209. LOGDEX unique identification number- 287

Operator/Company- HUMBLE

Well name- T. M. BROOKSHIRE 1

Location- Live Oak County, Texas

Township-Range-Section location- 16S-14E-6

API number- 42-297-

Log type- Induction Electrical

Logs available from 130 feet to 14112 feet.

Curve type(s) available-

Self Potential

Short Normal

Induction Conductivity

210. LOGDEX unique identification number- 288

Operator/Company- INTEGRAL ENERGY

Wel1 name- LENNOX-BURNS GAS UNIT A-1

Location- Live Oak County, Texas

Township-Range-Section location-14S-15E-8

API number- 42-297- 
Summary of LGGDEX Data Base

Log type- Induction Electrical

Logs available from 1000 feet to 11182 feet.

Curve type(s) available-

Self Potential

Induction Conductivity

Focussed Resistivity

211. LOGDEX unique identification number- 289

Operator/Company- TIDEWATER

We 11 name- MABEL W. HALL ESTATE A-1

Location- Live Oak County, Texas

Township-Range-Section location-15S-16E-4

Abstract/Tract/Block-A- 94

API number- 42-297- 2079

HEADER data base unique number- 12947

Date of completion- $3 / 20 / 65$

Well Classificattion code- O\&A

Log type- Induction Electrical

Logs available from 1150 feet to 11524 feet.

Curve type(s) available-

Self Potential

Short Norma 1

Induction Conductivity

212. LOGDEX unique identification number- 290

Operator/Company- STANDARD OIL COMPANY OF TEXAS

Well name- MRS. CLAY WEST BURNS 1

Location- Live Oak County, Texas

Township-Range-Section location- 15S-15E-5

Page 136 


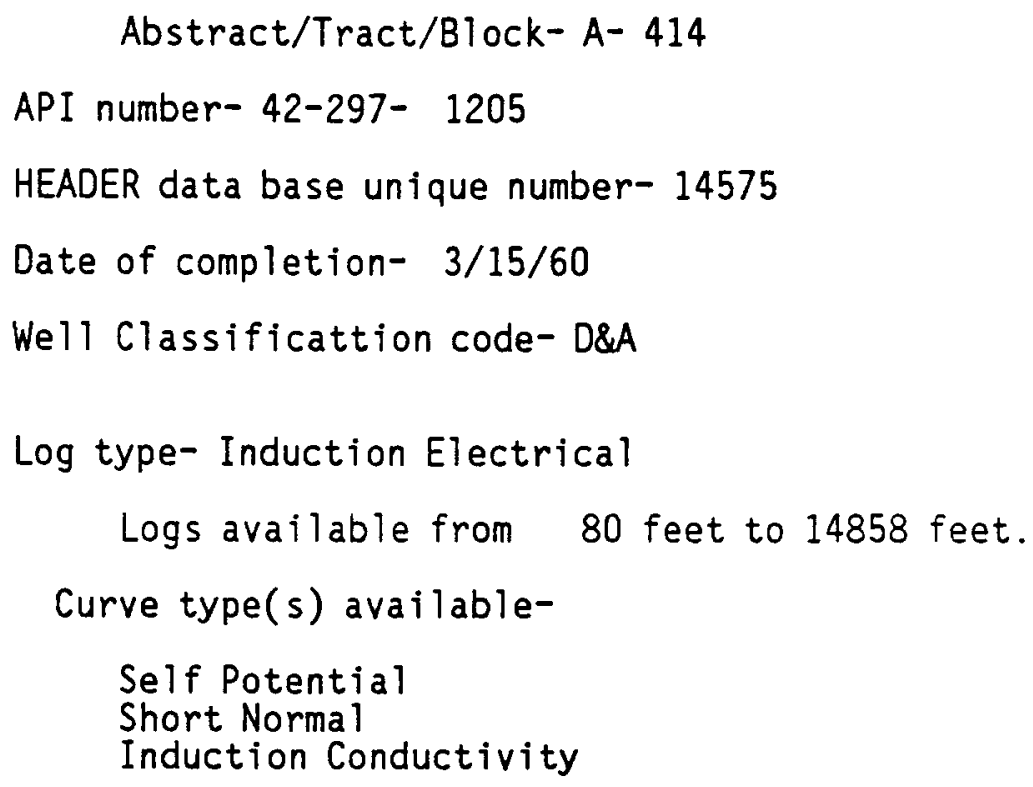

213. LOGDEX unique identification number- 291

Operator/Company- DAVIS

We 11 name- SANGER HEIRS 1

Location- Live Oak County, Texas

Township-Range-Section location-15S-14E-6

Abstract/Tract/Block-A- 860

API number- 42-297- 30852

HEADER data base unique number- 12895

Date of completion- $9 / 3 / 76$

Well Classificattion code- D\&A

Log type- Induction Electrical

Logs available from 1050 feet to 10574 feet.

Curve type(s) available-

Self Potential

Short Normal

Induction Conductivity 
214. LOGDEX unique identification number- 292

Operator/Company- M.P.S PRODUCTION -NORTH CENTRAL OIL - HARRY HURT

Wel1 name- DOUGHERTY ESTATE 1

Location- Live Oak County, Texas

Township-Range-Section location- 14S-14E-5

Abstract/Tract/Block-A-1010

API number- 42-297- 736

HEADER data base unique number- 12823

Date of completion- $8 / 26 / 61$

Well Classificattion code- GAS

Log type- Induction Electrical

Logs available from 730 feet to 8104 feet.

Curve type(s) available-

Self Potential

Short Normal

Lateral

Induction Conductivity

215. LOGDEX unique identification number- 293

Operator/Company- PAN AMERICAN

Well name- BROWN 1

Location- Harris County, Texas .

Township-Range-Section location- 1S-36E-8

API number- 42-201-

Log type- Induction Electrical

Logs available from 100 feet to 15500 feet.

Curve type(s) available- 
Self Potential

Short Normal

Induction Conductivity

Gamma Ray API Units

216. LOGDEX unique identification number- 294

Operator/Company- HOUSTON NATURAL GAS

We 11 name- HAMILL GA UNIT 21

Location- Harris County, Texas

Township-Range-Section location- 1S-37E-4

Abstract/Tract/Block-A- 262

API number- 42-201- 7853

HEADER data base unique number- 15038

Date of completion- $9 / 10 / 61$

Well Classificattion code- GAS

Log type- Induction Electrical

Logs available from 2558 feet to 14706 feet.

Curve type(s) available-

Self Potential

Short Normal

Induction Conductivity

Gamma Ray API Units

217. LOGDEX unique identification number- 295

Operator/Company- HUMBLE

Well name- T. E. SPARKS 1

Location-Waller County, Texas

Township-Range-Section location- 1S-36E-6

Abstract/Tract/Block- A- 174

API number- 42-473- 30027 
Summary of LOGOEX Data Base

HEADER data base unique number- 11083

Date of completion- $9 / 4 / 72$

Well Classificattion code- D\&A

Log type- Induction Electrical

Logs available from 230 feet to 11184 feet.

Curve type(s) available-

Self Potential

Induction Conductivity

Focussed Resistivity

218. LOGDEX unique identification number- 296

Operator/Company- STANDARD OIL COMPNAY OF TEXAS

We 11 name- H. J. LONGENBAUGH 1-1

Location- Harris County, Texas

Township-Range-Section location- 1S-35E-4

Abstract/Tract/Block-A- 432

API number- 42-201- 3941

HEADER data base unique number- 11088

Date of completion- 4/4/61

We11 Classificattion code- GAS

Log type- Induction Electrical

Logs available from 1720 feet to 15010 feet.

Curve type(s) available-

Self Potential

Induction Conductivity

Focussed Resistivity

219. LOGDEX unique identification number- 297 


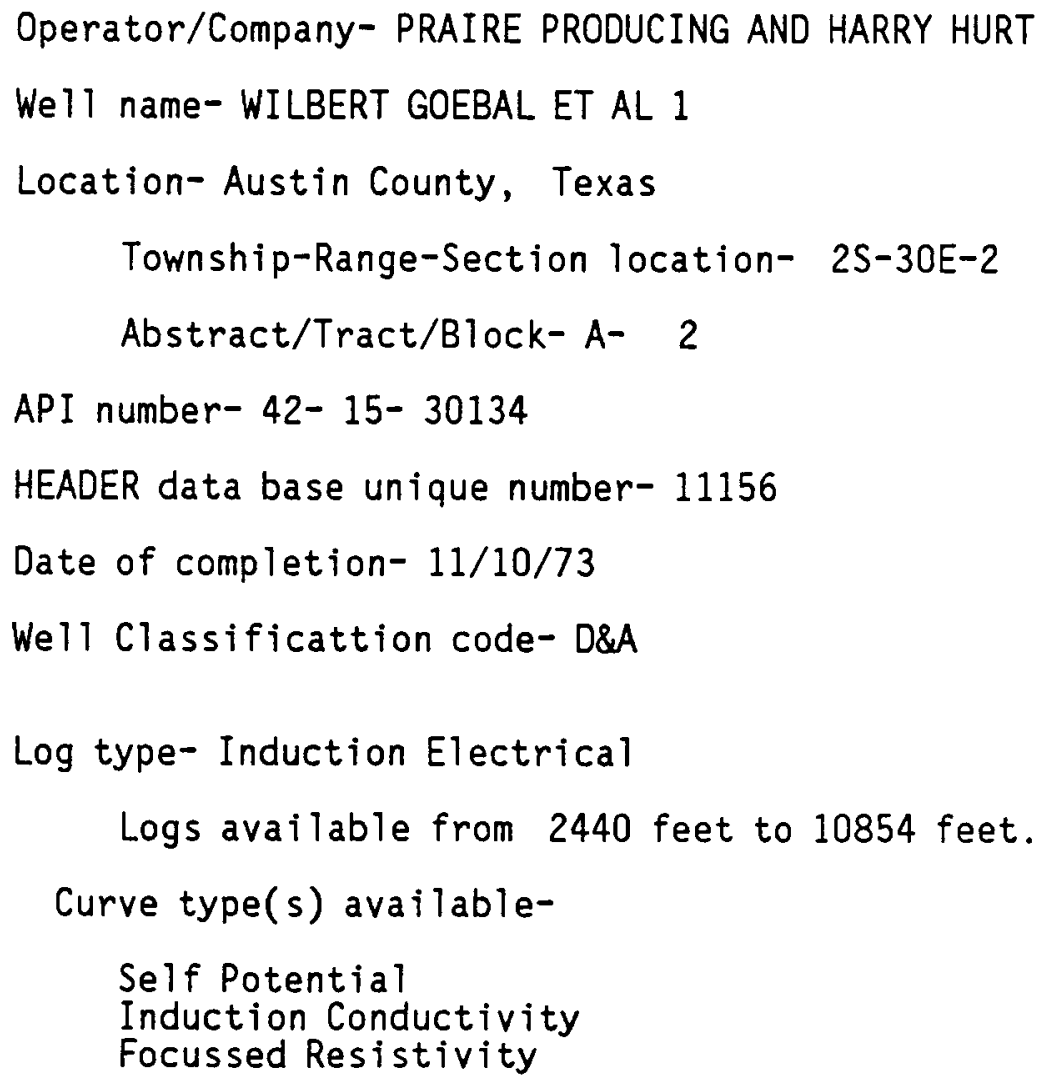

220. LOGDEX unique identification number- 298

Operator/Company- SHELL ET AL

We 11 name- SEALY GAS UNIT 1

Location-Austin County, Texas

Township-Range-Section location- 2S-31E-9

API number- 42- $15-$

Log type- Induction Electrical

Logs available from 2530 feet to 15512 feet.

Curve type(s) available-

Self Potential

Short Normal

Induction Conductivity 
221. LOGDEX unique identification number- 299

Operator/Company- KING RESOURCES

We11 name- MARY LYLE 1

Location-Colorado County, Texas

Township-Range-Section location- 3S-29E-8

API number- 42-89-

Log type- Induction Electrical

Logs available from 2550 feet to 9608 feet.

Curve type(s) available-

Self Potential

Short Norma 1

Induction Conductivity

222. LOGDEX unique identification number- 300

Operator/Company- NEWMONT OIL AND TIDEWATER OIL

We 11 name- ANNABELL EVERETT 1

Location-Colorado County, Texas

Township-Range-Section location- 3S-30E-5

Abstract/Tract/Block- A- 687

API number- 42- 89- 904

HEADER data base unique number- 11223

Date of completion- 6/13/67

Well Classificattion code- D\&A

Log type- Induction Electrical

Logs available from 4000 feet to 12156 feet.

Curve type(s) available- 


\author{
Self Potential \\ Short Normal \\ Induction Conductivity
}

223. LOGDEX unique identification number- 301

Operator/Company- SUNDANCE OIL, MAY PETROLEUM AND STUARCO OIL

Well name- BEST 2

Location-Austin County, Texas

Township-Range-Section location- 3S-31E-5

API number- 42- 15-

Log type- Induction Electrical

Logs available from 2750 feet to 13774 feet.

Curve type(s) available-

Self Potential

Induction Conductivity

Focussed Resistivity

224. LOGDEX unique identification number- 302

Operator/Company- HALBOUTY MICHEL T

Well name- FOSTER-GULF 1

Location-Montgomery County, Texas

Township-Range-Section location- 3N-39E-5

Abstract/Tract/Block-A- 310

API number- 42-339- 25

HEADER data base unique number- 14579

Date of completion- 9/9/59

Well Classificattion code- GAS

Log type- Induction Electrical 
Logs available from 1850 feet to 10954 feet.

Curve type(s) available-

Self Potential

Short Norma 1

Induction Conductivity

225. LOGDEX unique identification number- 303

Operator/Company- MORAN

We 11 name- RANDALL CARTWRIGHT 1

Location-Montgomery County, Texas

Township-Range-Section location- 3N-37E-4

Abstract/Tract/81ock-A- 289

API number- 42-339- 30078

HEADER data base unique number- 11418

Date of completion-11/8/72

Well Classificattion code- D\&A

Log type- Induction Electrical

Logs available from 2410 feet to 12440 feet.

Curve type(s) available-

Self Potential

Induction Conductivity

Focussed Resistivity

226. LOGDEX unique identification number- 304

Operator/Company- MCCULLOCH AND VENUS

Well name- E. G. FROST ESTATE 1

Location- Montgomery County, Texas

Township-Range-Section location- 3N-36E-8

Abstract/Tract/Block-A- 18

Page 144 
API number- 42-339- 30079

HEADER data base unique number- 11138

Date of completion- $6 / 8 / 73$

Well Classificattion code- D\&A

Log type- Induction Electrical

Logs available from 2720 feet to 14002 feet.

Curve type(s) available-

Self Potential

Induction Conductivity

Focussed Resistivity

227. LOGDEX unique identification number- 305

Operator/Company-MOBIL

We11 name- CHESTERVILLE 8

Location-Colorado County, Texas

Township-Range-Section location- 4S-31E-3

Abstract/Tract/Block- A- 707

API number- 42- 89- 909

HEADER data base unique number- 11669

Date of completion- 8/10/67

Well Classificattion code- GAS

Log type- Induction Electrical

Logs available from 1560 feet to 9600 feet.

Curve type(s) available-

Self Potential

Short Normal

Induction Conductivity 
228. LOGDEX unique identification number- 306

Operator/Company- TEX-STAR AND HARKINS

We11 name- T. P. HALEY TRUST 1

Location- Colorado County, Texas

Township-Range-Section location- 4S-30E-5

Abstract/Tract/Block-A- 239

API number- 42- 89- 289

HEADER data base unique number- 11658

Date of completion- 12/17/61

Wel1 Classificattion code- D\&A

Log type- Induction Electrical

Logs available from 1030 feet to 10022 feet.

Curve type(s) available-

Self Potential

Short Normal

Induction Conductivity

229. LOGDEX unique identification number- 307

Operator/Company- TEXAS OIL AND GAS

Wel1 name- OSCAR BUNGE 1

Location- Colorado County, Texas

Township-Range-Section location- 4S-29E-8

Abstract/Tract/Block-A- 48 .

API number- 42- $89-30423$

HEADER data base unique number- 15573

Date of completion- $5 / 7 / 75$

Well Classificattion code- GAS

Log type- Induction Electrical 


\author{
Logs available from 1300 feet to 10572 feet. \\ Curve type(s) available- \\ Self Potential \\ Short Normal \\ Induction Conductivity
}

230. LOGDEX unique identification number- 308

Operator/Company- CRESCENT

We 11 name- JAUN G. BENAVIDES 2

Location- Zapata County, Texas

Township-Range-Section location-26S-6E-8

Abstract/Tract/Block-A- 71

API number- 42-507- 1998

HEADER data base unique number- 13606

Date of completion- 6/29/62

Well Classificattion code- GAS

Log type- Induction Electrical

Logs avajlable from 200 feet to 11932 feet.

Curve type(s) available-

Self Potential

Short Normal

Induction Conductivity

231. LOGDEX unique identification number- 309

Operator/Company- TEXAS OIL AND GAS

We 11 name- 0. V. GUERRA "L" 1

Location- Zapata County, Texas

Township-Range-Section location- 26S-7E-8

API number- 42-507- 
Log type- Induction Electrical

Logs available from 1000 feet to 9000 feet.

Curve type(s) available-

Self Potential

Short Normal

Induction Conductivity

232. LOGDEX unique identification number- 310

Operator/Company- HUGO ALLEN

Wel1 name- GARCIA-VILLAREAL 1

Location-Webb County, Texas

Township-Range-Section location-20S-9E-9

API number- 42-479-

Log type- Induction Electrical

Logs available from 1450 feet to 8514 feet.

Curve type(s) available-

Self Potential

Short Normal

Induction Conductivity

233. LOGDEX unique identification number- 311

Operator/Company-MOBIL

We11 name- BRENNAN BENAVIDES "B" 1

Location- Duval County, Texas

Township-Range-Section location- 21S-10E-7

API number- 42-131- 
Log type- Induction Electrical

Logs available from 100 feet to 13652 feet.

Curve type(s) available-

Self Potential

Short Normal

Induction Conductivity

Gamma Ray API Units

234. LOGDEX unique identification number- 312

Operator/Company- ATLANTIC REFINING

We 11 name- E. GARCIA ESTATE "A" 1

Location-Webb County, Texas

Township-Range-Section location-22S- 9E-9

Abstract/Tract/Block- A-1393

API number- 42-479- 4661

HEADER data base unique number- 11714

Date of completion- 7/15/64

Well Classificattion code- D\&A

Log type- Induction Electrical

Logs available from 100 feet to 10008 feet.

Curve type(s) available-

Self Potential

Short Normal

Induction Conductivity

235. LOGDEX unique identification number- 313

Operator/Company- COASTAL STATES

We 11 name- V. L. PUIG 1

Location-Webb County, Texas 
Summary of LOGDEX Data Base

\author{
Township-Range-Section location-22S-9E-2 \\ Abstract/Tract/Block- A-1996 \\ API number- 42-479- 4703 \\ HEADER data base unique number- 14637 \\ Date of completion- 2/ $1 / 65$ \\ We11 Classificattion code- D\&A \\ Log type- Velocity \\ Logs available from 616 feet to 10700 feet. \\ Curve type(s) available- \\ Self Potential \\ Acoustic Compensated \\ Log type- Induction Electrical \\ Logs available from 610 feet to 11664 feet. \\ Curve type(s) available- \\ Self Potential \\ Short Normal \\ Induction Conductivity
}

236. LOGDEX unique identification number- 314

Operator/Company- ATLANTIC RICHFIELD

We 11 name- M. MCLEAN TRUST "C" 2

Location- Jim Hogg County, Texas

Township-Range-Section location- 22S-10E-9

API number- 42-247-

Log type- Induction Electrical

Logs available from 720 feet to 16030 feet.

Curve type(s) available-

Self Potential

Page 150 


\author{
Short Normal \\ Long Normal \\ Lateral \\ Induction Conductivity
}

237. LOGDEX unique identification number- 316

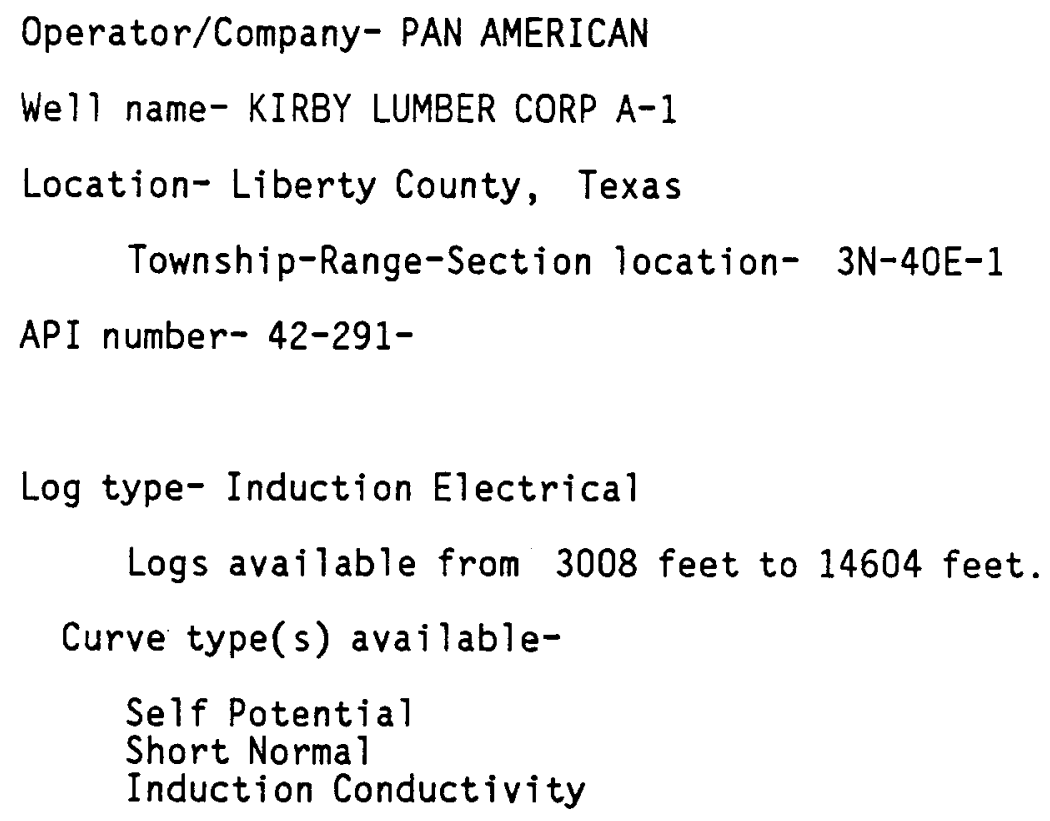

238. LOGDEX unique identification number- 317

Operator/Company- SUNDANCE

We11 name- WIRT DAVIS 1

Location- Liberty County, Texas

Township-Range-Section locatjon- $3 \mathrm{~N}-40 \mathrm{E}-2$

Abstract/Tract/Block- A- 304

API number- 42-291- 30262

HEADER data base unique number- 14670

Date of completion- 1/20/72

Well Classificattion code- D\&A 
Summary of LOGDEX Data Base

Log type- Induction Electrical

Logs available from 2050 feet to 11018 feet.

Curve type(s) available-

Self Potential

Induction Conductivity

Focussed Resistivity

239. LOGDEX unique identification number- 318

Operator/Company- PRAIRE PRODUCING AND COVEST ENERGY

Wel1 name- GIBBS BROS ET AL 1

Location-San Jacinto County, Texas

Township-Range-Section location- 4N-39E-3

Abstract/Tract/Block- A- 40

API number- 42-407- 2883

HEADER data base unique number- 11473

Date of completion- $1 / 7 / 59$

Well Classificattion code- D\&A

Log type- Induction Electrical

Logs available from 1830 feet to 11236 feet.

Curve type(s) available-

Self Potential

Induction Conductivity

Focussed Resistivity

240. LOGDEX unique identification number- 319

Operator/Company- AMOCO

We 11 name- W. W. LANGHAM 1

Location-San Jacinto County, Texas

Township-Range-Section location- 5N-41E-9

Page 152 


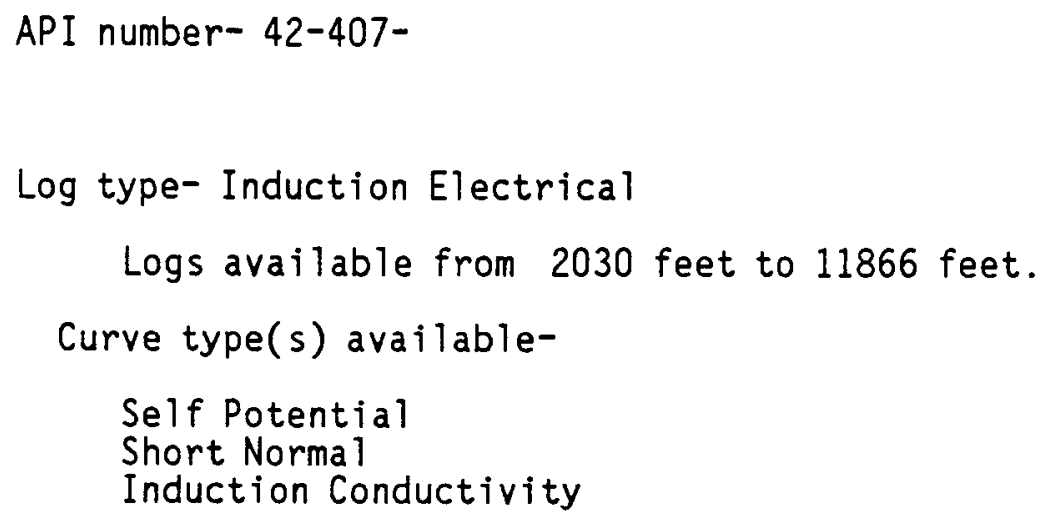

241. LOGDEX unique identification number- 320

Operator/Company- VIKING DRILLING AND RODNEY DE LANGE

Well name- LANGRAM GAS UNIT 1

Location- San Jacinto County, Texas

Township-Range-Section location- 5N-40E-6

API number- 42-407-

Log type- Induction Electrical

Logs available from 1450 feet to 10530 feet.

Curve type(s) available-

Self Potential

Short Normal

Induction Conductivity

Induction Resistivity

242. LOGDEX unique identification number- 321

Operator/Company - STANDARD OF TEXAS

We 11 name- FOSTER LUMBER COMPANY 1

Location- San Jacinto County, Texas

Township-Range-Section location- $5 \mathrm{~N}-39 \mathrm{E}-7$

Abstract/Tract/Block-A- 292 


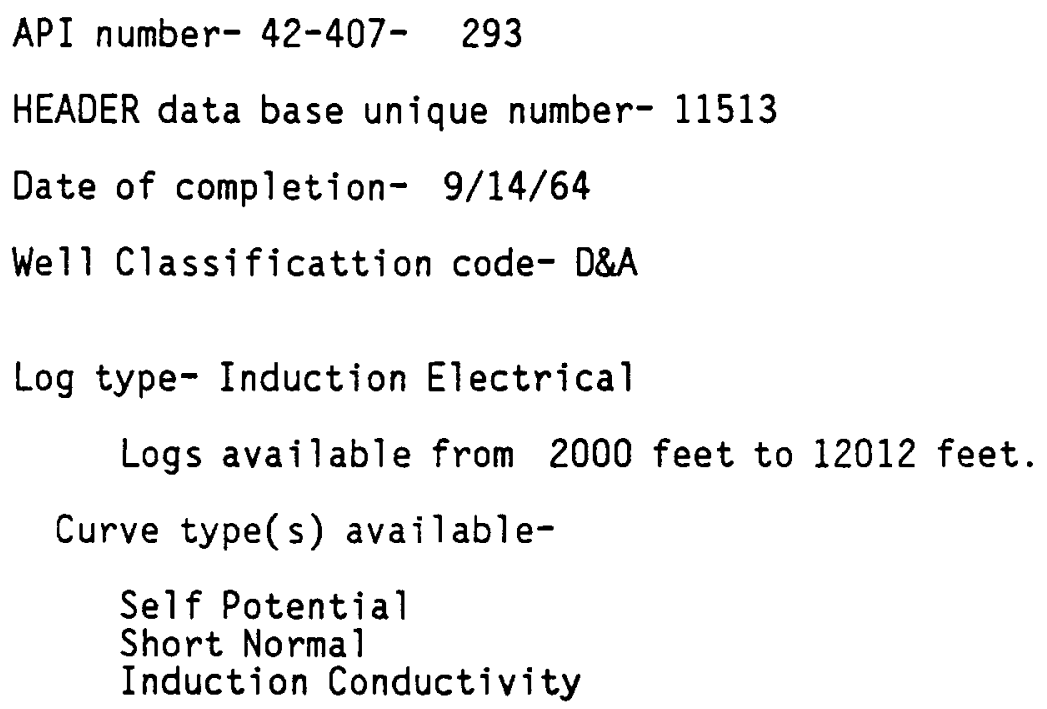

243. LOGDEX unique identification number- 322

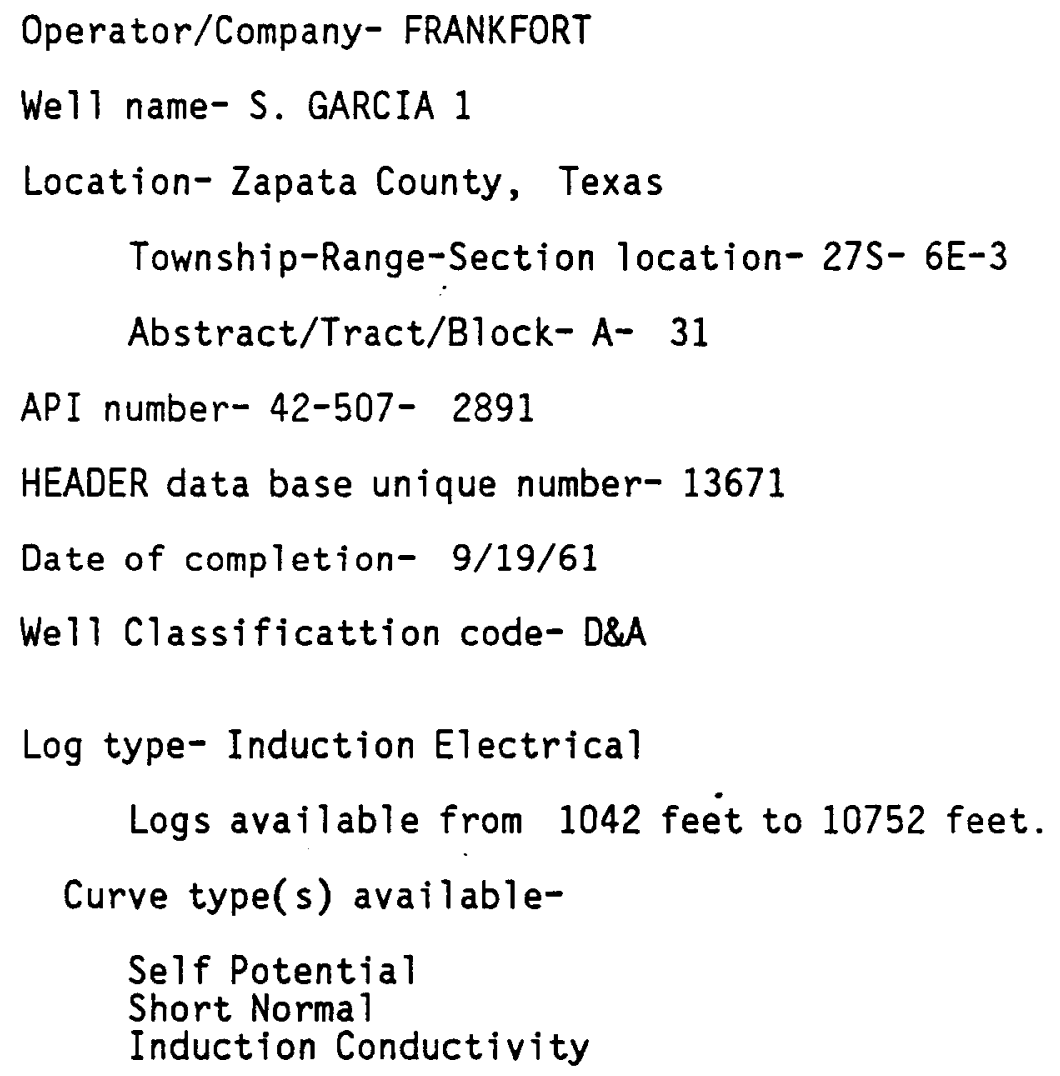


244. LOGDEX unique identification number- 323

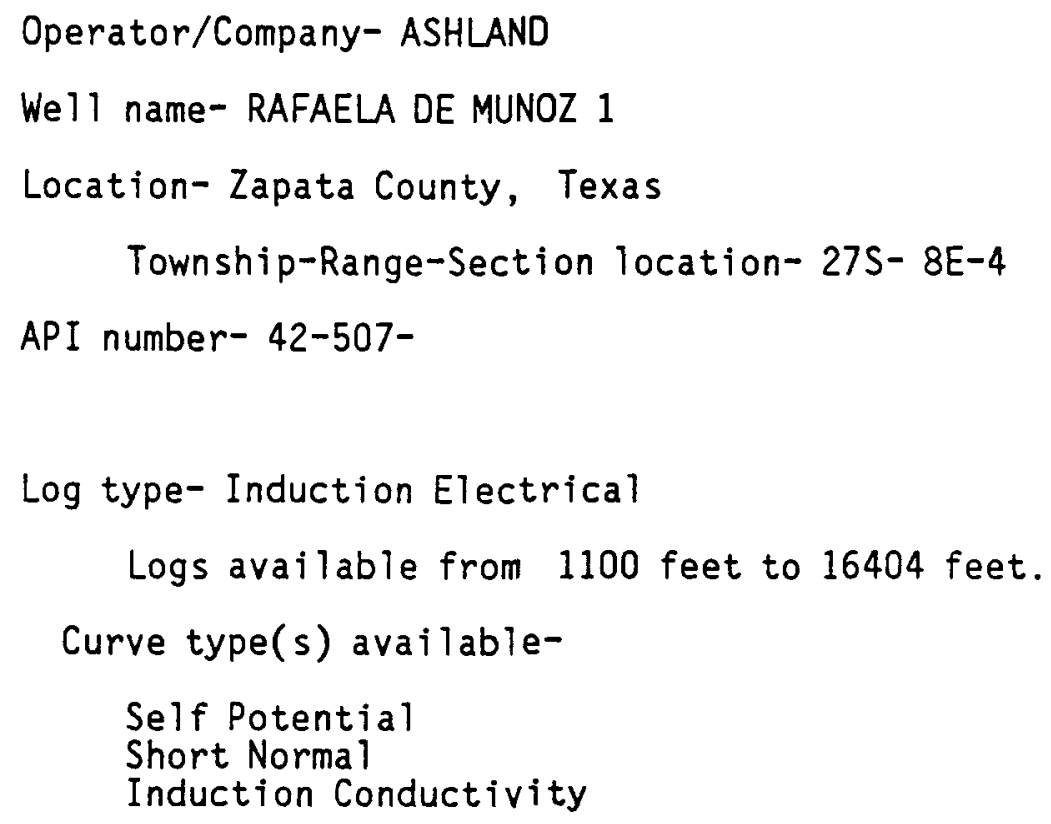

245. LOGDEX unique identification number- 324

Operator/Company- ROBERT MOSBACHER

We 11 name- T. E. FULBRIGHT 1

Location- Jim Hogg County, Texas

Township-Range-Section location-22S-9E-8

API number- 42-247-

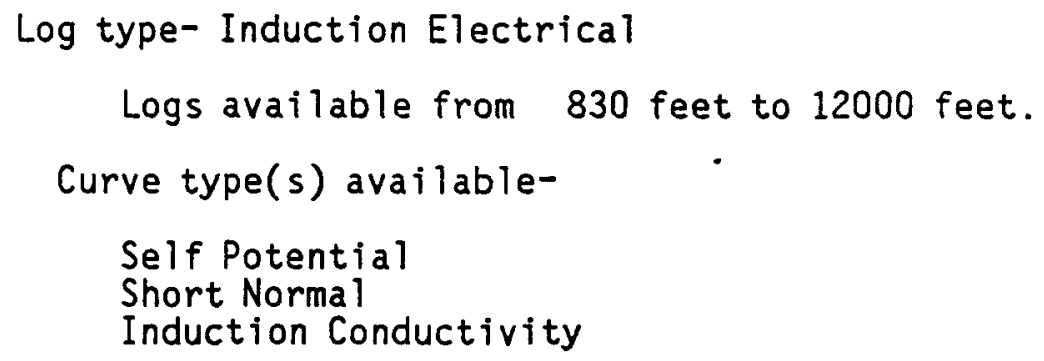

246. LOGDEX unique identification number- 325 
Summary of LOGDEX Data Base

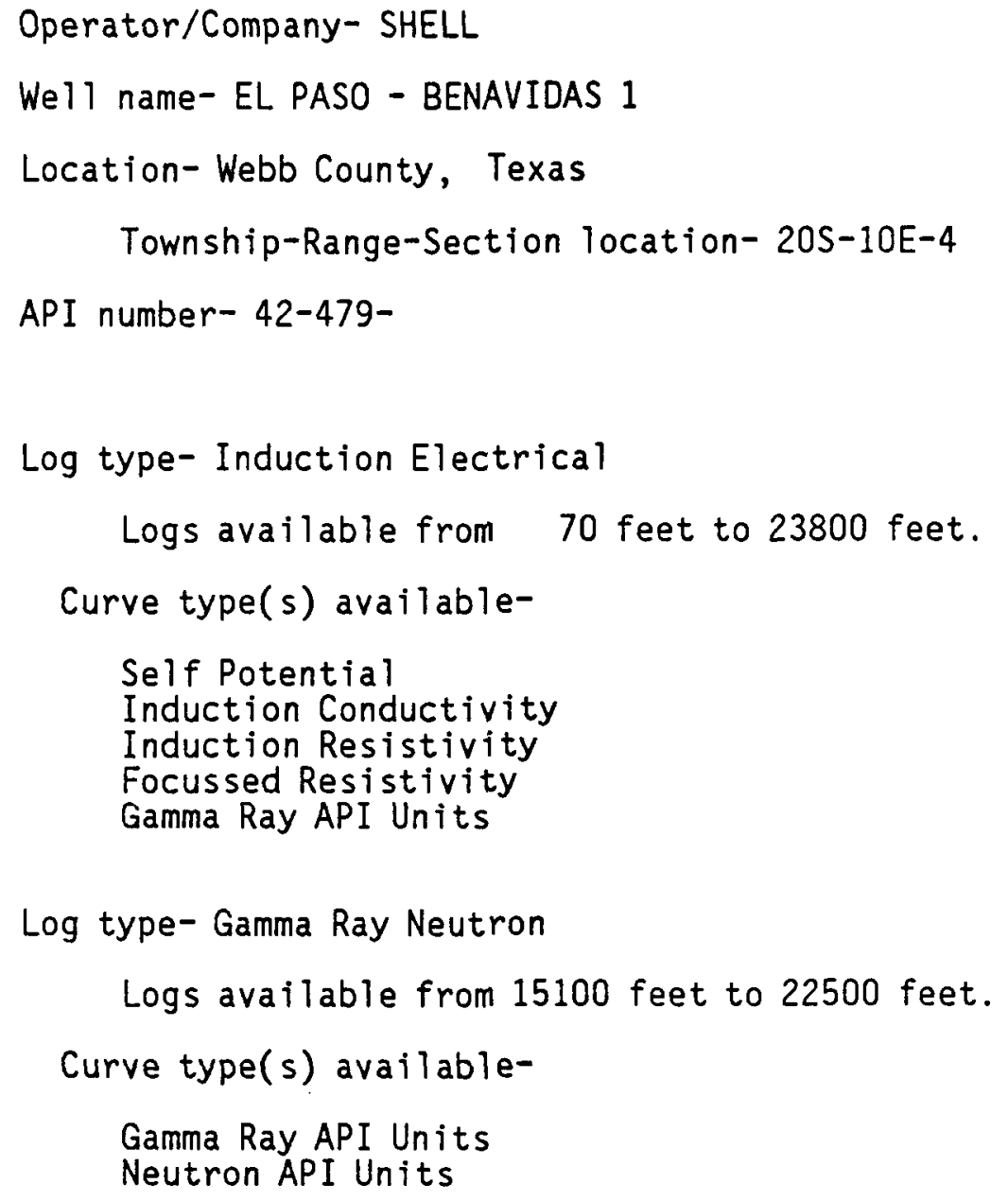

Log type- Gamma Ray Neutron

Logs available from 15100 feet to 22500 feet.

Curve type(s) available-

Gamma Ray API Units

Neutron API Units

247. LOGDEX unique identification number- 326

Operator/Company- GETTY, MISSION AND OHIO

We 11 name- STATE TRACT 1251

Location-Aransas County, Texas. Township-Range-Section location- 16S-24E-6

Abstract/Tract/Block- T- 125

API number- 42- 7- 542

HEADER data base unique number- 14741

Date of completion- 9/19/58

Well Classificattion code- GAS 
Log type- Induction Electrical

Logs available from 320 feet to 9832 feet.

Curve type(s) available-

Self Potential

Short Norma ]

Induction Conductivity

248. LOGDEX unique identification number- 327

Operator/Company- HUMBLE

We 11 name- HARBOR ISLAND GAS UNIT 1

Location- Nueces County, Texas

Township-Range-Section location- 18S-24E-3

Abstract/Tract/Block- T- 312

API number- 42-355- 6552

HEADER data base unique number- 11673

Date of completion-12/24/67

Well Classificattion code- D\&A

Log type- Induction Electrical

Logs available from 2582 feet to 15412 feet.

Curve type(s) available-

Self Potential

Short Normal

Induction Conductivity

249. LOGDEX unique identification number- 328

Operator/Company- ARNOLD O MORGAN

We11 name- BESSIE K. WELDER ET AL A-1

Location-San Patricio County, Texas

Township-Range-Section location-18S-23E-3 
Summary of LOGDEX Data Base

\author{
Abstract/Tract/Block-A- 292 \\ API number- $42-409-4160$ \\ HEADER data base unique number- 13304 \\ Date of completion- 1/4/67 \\ Well Classificattion code- D\&A \\ Log type- Induction Electrical \\ Logs available from 1600 feet to 12520 feet. \\ Curve type(s) available- \\ Self Potential \\ Short Normal \\ Induction Conductivity
}

250. LOGDEX unique identification number- 329

Operator/Company- OHIO

We 11 name- JEAN M. DAVIS 1

Location-San Patricio County, Texas

Township-Range-Section location-17S-23E-4

Abstract/Tract/Block-A- 132

API number- 42-409- 2239

HEADER data base unique number- 13163

Date of completion- 2/9/60

Well Classificattion code- GAS

Log type- Induction Electrical

Logs available from 200 feet to 12450 feet.

Curve type(s) available-

Self Potential

Short Normal

Induction Conductivity

Page 158 
251. LOGDEX unique identification number- 330

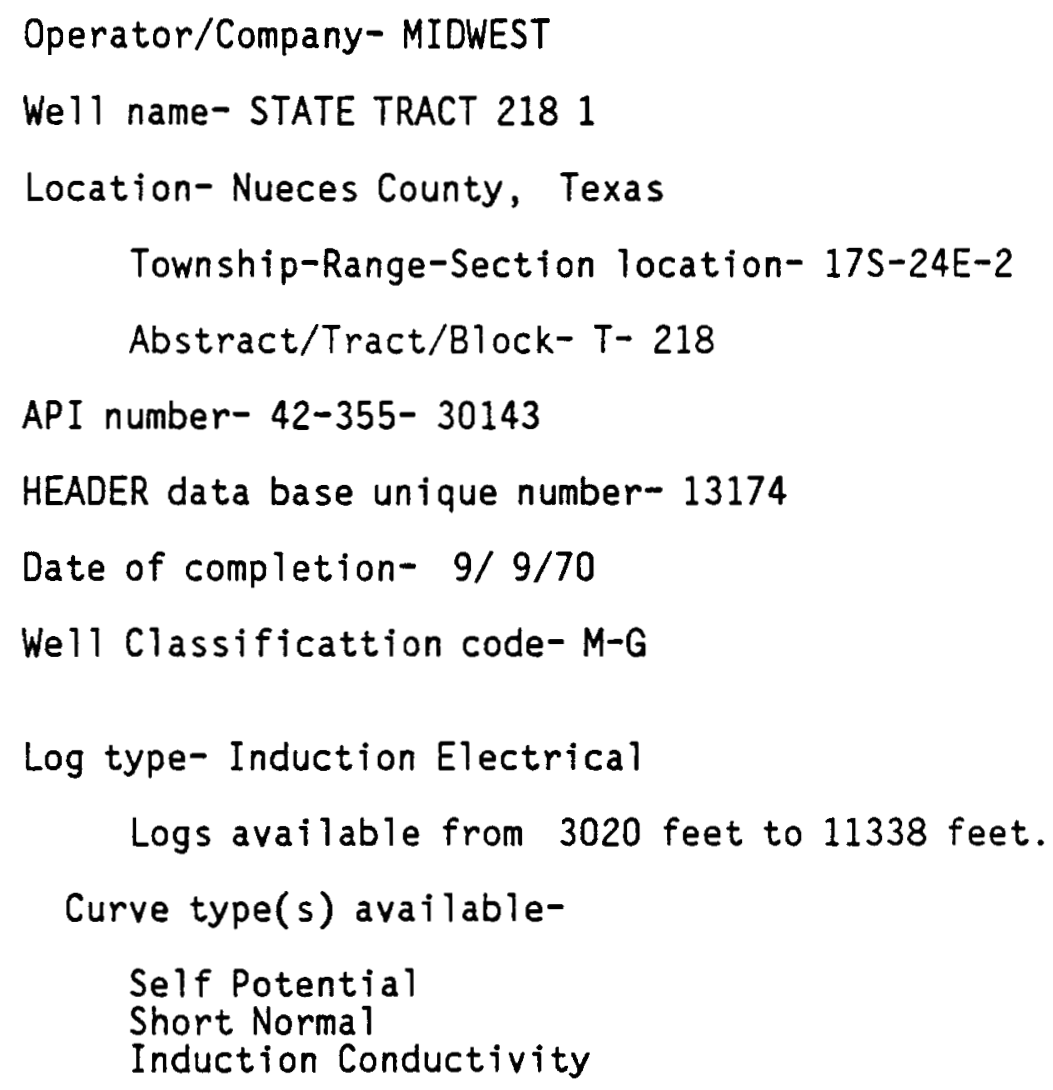

252. LOGDEX unique identification number- 331

Operator/Company-GETTY

We 11 name- AMERICAN LIBERTY OIL COMPANY 1

Location-Calhoun County, Texas

Township-Range-Section location-16S-26E-2

Abstract/Tract/Block-A- 203

API number- 42- 57- 30050

HEADER data base unique number- 11629

Date of completion- $5 / 9 / 70$

Well Classificattion code- D\&A 
Summary of LOGDEX Data Base

Log type- Induction Electrical

Logs available from 1520 feet to 11516 feet.

Curve type(s) available-

Self Potential

Short Normal

Induction Conductivity

Gamma Ray API Units

253. LOGDEX unique identification number- 332

Operator/Company- SUNRAY MID-CONTINENT

We 11 name- STATE TRACT 961

Location-Aransas County, Texas

Township-Range-Section location- 16S-25E-3

Abstract/Tract/Block- T- 96

API number- 42- 7- 98

HEADER data base unique number- 13085

Date of completion- $3 / 30 / 59$

Well Classificattion code- D\&A

Log type- Induction Electrical

Logs available from 2016 feet to 11860 feet.

Curve type(s) available-

Self Potential

Short Normal

Induction Conductivity

254. LOGDEX unique identification number- 333

Operator/Company- MIDWEST

We 11 name- STATE TRACT 1221

Location-Aransas County, Texas 
Township-Range-Section location-16S-23E-6

Abstract/Tract/Block- T- 122

API number- 42- 7- 929

HEADER data base unique number- 13048

Date of completion- $8 / 14 / 68$

Well Classificattion code- MOG

Log type- Induction Electrical

Logs available from 1290 feet to 10700 feet.

Curve type(s) available-

Self Potential

Short Norma 7

Induction Conductivity

255. LOGDEX unique identification number- 334

Operator/Company- GULF

Wel1 name-MUELLER 1

Location-DeWitt County, Texas

Township-Range-Section location- 8S-21E-1

API number- 42-123-

Log type- Velocity

Logs available from 100 feet to 13988 feet.

Curve type(s) available-

Self Potential

Acoustic Compensated

Caliper

256. LOGDEX unique identification number- 335

Operator/Company- HUGHES \& HUGHES 


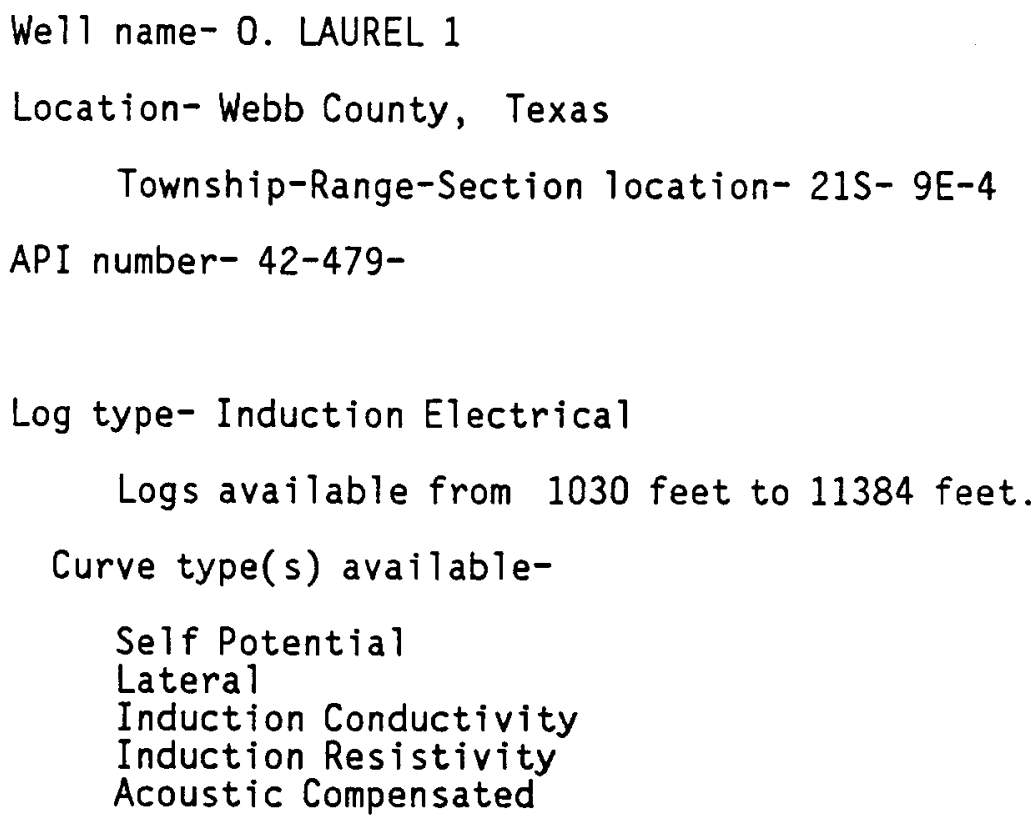

257. LOGDEX unique identification number- 336

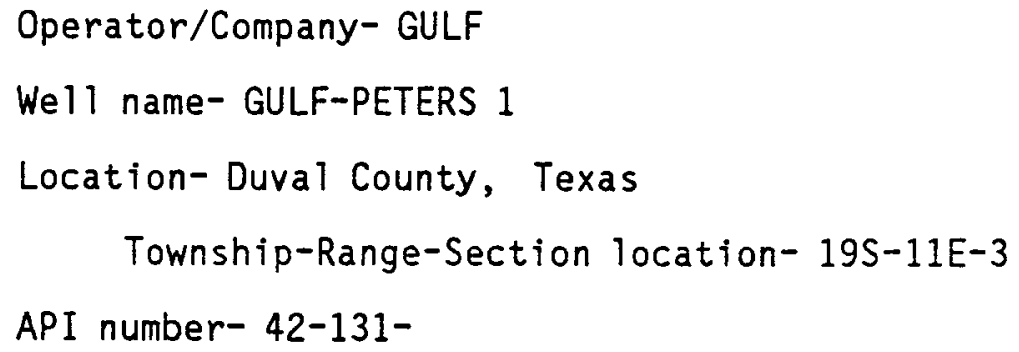


Acoustic Compensated

258. LOGDEX unique identification number- 337

Operator/Company- ATLANTIC RICHFIELD

Well name- RHODES 541

Location-McMullen County, Texas

Township-Range-Section location-16S-13E-1

API number- 42-311-

Log type- Induction Electrical

Logs available from 7300 feet to 13532 feet.

Curve type(s) available-

Self Potential

Short Normal

Induction Conductivity

Gamma Ray API Units

Log type- Density

Logs available from 8300 feet to 13452 feet.

Curve type(s) available-

Density API Units

Gamma Ray API Units

Caliper

Monitor

259. LOGDEX unique identification number- 338

Operator/Company-SHELL

We 11 name- L. WEATHERBY 1

Location- Duval County, Texas

Township-Range-Section location- 18S-12E-6

API number- 42-131- 


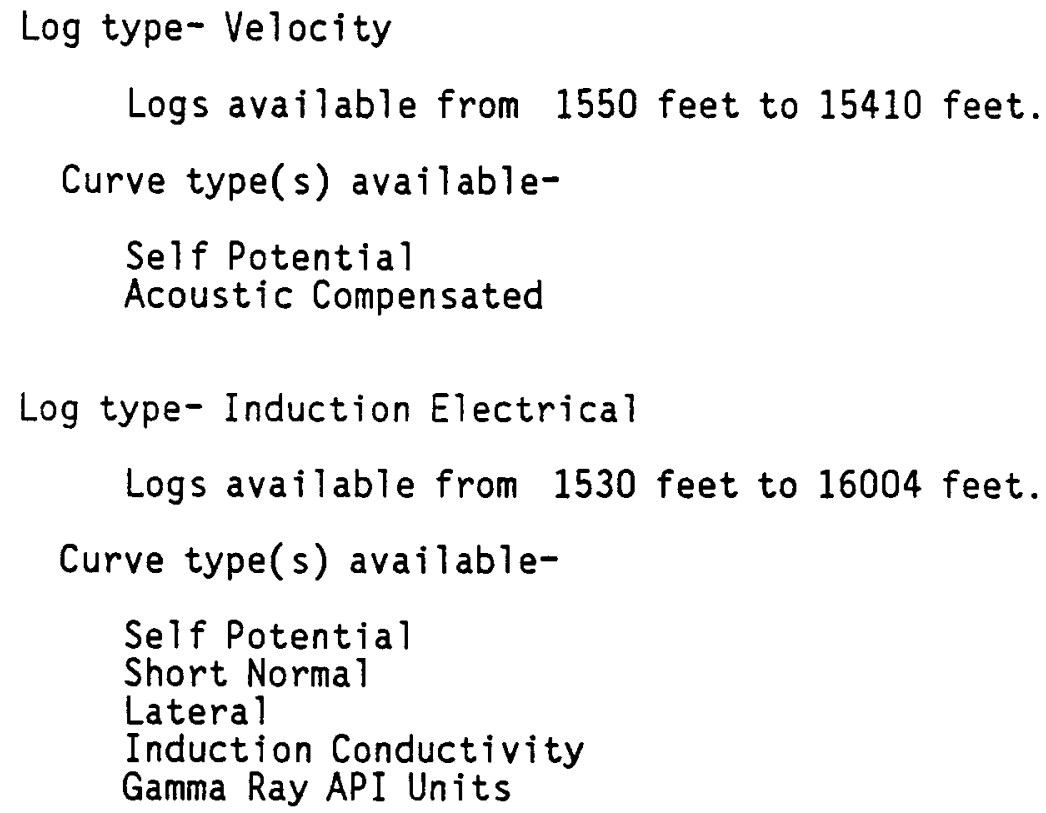

260. LOGDEX unique identification number- 339

Operator/Company- KILLAM \& HURD LTD.

We 11 name- B. LOPEZ 1

Location-Webb County, Texas

Township-Range-Section location-22S-9E-3

API number- 42-479-

Log type- Induction Electrical

Logs available from 400 feet to 11084 feet.

Curve type(s) available-

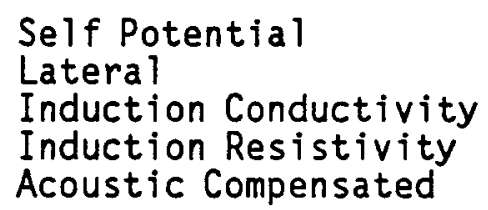


261. LOGDEX unique identification number- 340

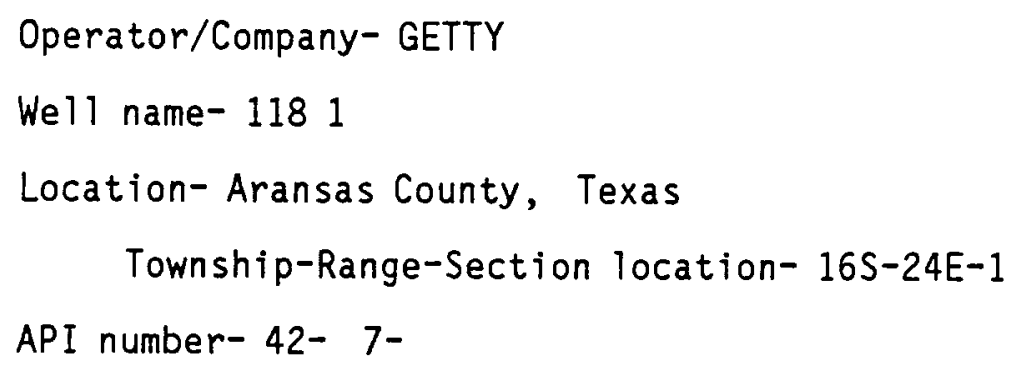

262. LOGDEX unique identification number- 341

Operator/Company-CITIES SERVICE

Well name- BOGGAN 1

Location- Nueces County, Texas

Township-Range-Section location-20S-19E-1

API number- 42-355-

Log type- Velocity

Logs available from 2000 feet to 13104 feet.

Curve type(s) available-

Self Potential

Acoustic Compensated

263. LOGDEX unique identification number- 342

Operator/Company- PAN AMERICAN 


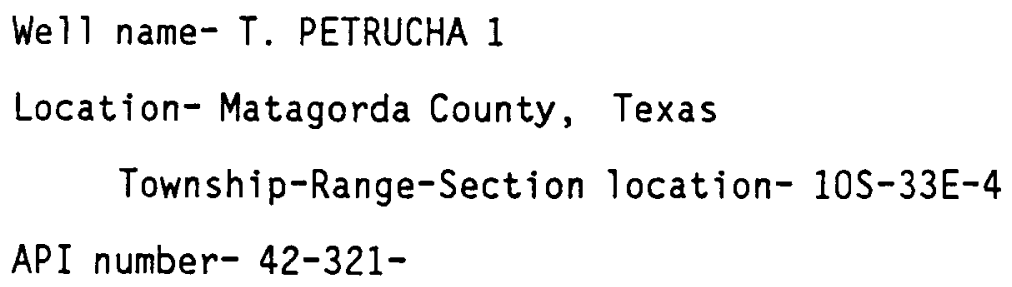

264. LOGDEX unique identification number- 343

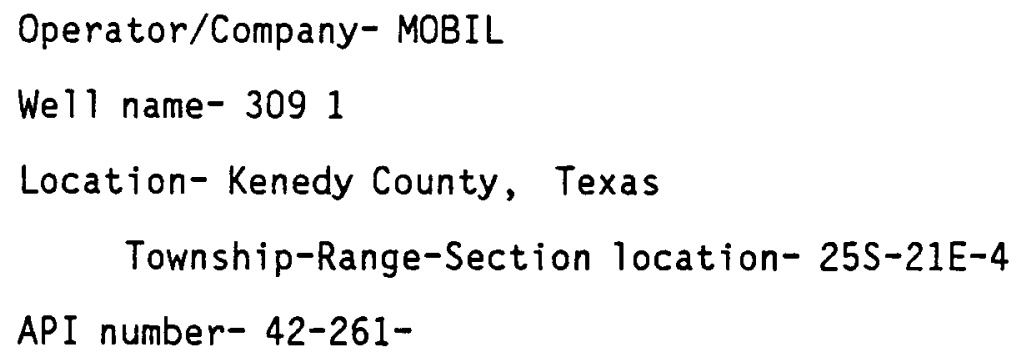

265. LOGDEX unique identification number- 344

Operator/Company- STANDARD

We 11 name- W. MOOTHART 1

Location-Cameron County, Texas 
Township-Range-Section location- 32S-18E-1

API number- 42- 61-

Log type- Velocity

Logs available from 2000 feet to 16002 feet.

Curve type(s) available-

Self Potential

Acoustic Compensated

266. LOGDEX unique identification number- 345

Operator/Company- CHEVRON

Well name- J. RODRIGUEZ 1

Location- Cameron County, Texas

Township-Range-Section location- 31S-20E-5

API number- 42- 61-

Log type-Velocity

Logs available from 300 feet to 18500 feet.

Curve type(s) available-

Self Potential

Acoustic Compensated

267. LOGDEX unique identification number- 346

Operator/Company-SHELL

Well name- J. ALVARADO 1

Location-Bee County, Texas

Township-Range-Section location-12S-17E-3

API number- 42- 25- 
Summary of LOGDEX Data Base

\author{
Log type- Velocity \\ Logs available from ,3530 feet to 15800 feet. \\ Curve type(s) available- \\ Self Potential \\ Acoustic Compensated
}

268. LOGDEX unique identification number- 347

Operator/Company- PAN AMERICAN

Well name- D. BROWN 1
Location- Harris County, Texas
Township-Range-Section location- $15-36 E-8$
API number- $42-201-$
Log type- Velocity
Logs available from 150 feet to 15524 feet.
Curve type(s) available-
Self Potential
Acoustic Compensated
Gamma Ray API Units

269. LOGDEX unique identification number- 348

Operator/Company- SHELL

We 11 name- 0. FRIEDRICHS 1

Location- Goliad County, Texas

Township-Range-Section location-11S-22E-9

API number- 42-175-

Log type- Velocity

Logs available from 100 feet to 16000 feet. 
Curve type(s) available-

Self Potential

Acoustic Compensated

270. LOGDEX unique identification number- 349

Operator/Company-SOHIO \& SKELLY

Well name- $P$. STOCK 1

Location-Lavaca County, Texas

Township-Range-Section location- 45-25E-8

API number- 42-285-

Log type- Velocity

Logs available from 1810 feet to 14000 feet.

Curve type(s) available-

Self Potential

Acoustic Compensated

271. LOGDEX unique identification number- 350

Operator/Company- SHELL

Well name- KIRBY LUMBER 1

Location- Liberty County, Texas

Township-Range-Section location- $2 \mathrm{~N}-41 \mathrm{E}-5$

API number- 42-291-

Log type- Velocity

Logs available from 2550 feet to 17150 feet.

Curve type(s) available-

Acoustic Compensated

Gamma Ray API Units 
272. LOGDEX unique identification number- 351

Operator/Company- AMOCO

Well name- PENNY 1

Location-Matagorda County, Texas

Township-Range-Section location-10S-32E-6

API number- 42-321-

Log type- Velocity

Logs available from 2024 feet to 13980 feet.

- Curve type(s) available-

Self Potential

Acoustic Compensated

273. LOGDEX unique identification number- 352

Operator/Company- TENNECO

We 11 name- W. MCCAMBELL 1

Location- San Patricio County, Texas

Township-Range-Section location- 17S-23E-5

API number- 42-409-

Log type- Velocity

Logs available from 6500 feet to 14052 feet.

Curve type(s) available-

Self Potential

Acoustic Compensated

274. LOGDEX unique identification number- 353

Page 170 
Operator/Company- SHELL

Well name- L. PENWELL 1

Location- Duval County, Texas

Township-Range-Section location- 18S-12E-5

API number- 42-131-

Log type- Velocity

Logs available from 1050 feet to 12900 feet.

Curve type(s) available-

Self Potential

Acoustic Compensated

275. LOGDEX unique identification number- 354

Operator/Company- HUMBLE

We 11 name- J. KENEDY 4

Location- Kenedy County, Texas

Township-Range-Section location- 24S-19E-2

API number- 42-261-

Log type- Velocity

Logs available from 100 feet to 20004 feet.

Curve type(s) available-

Self Potential

Acoustic Compensated

276. LOGDEX unique identification number- 355

Operator/Company- HUMBLE

We 11 name- HUMBLE - MARTINEZ III 1 
Summary of LOGDEX Data Base

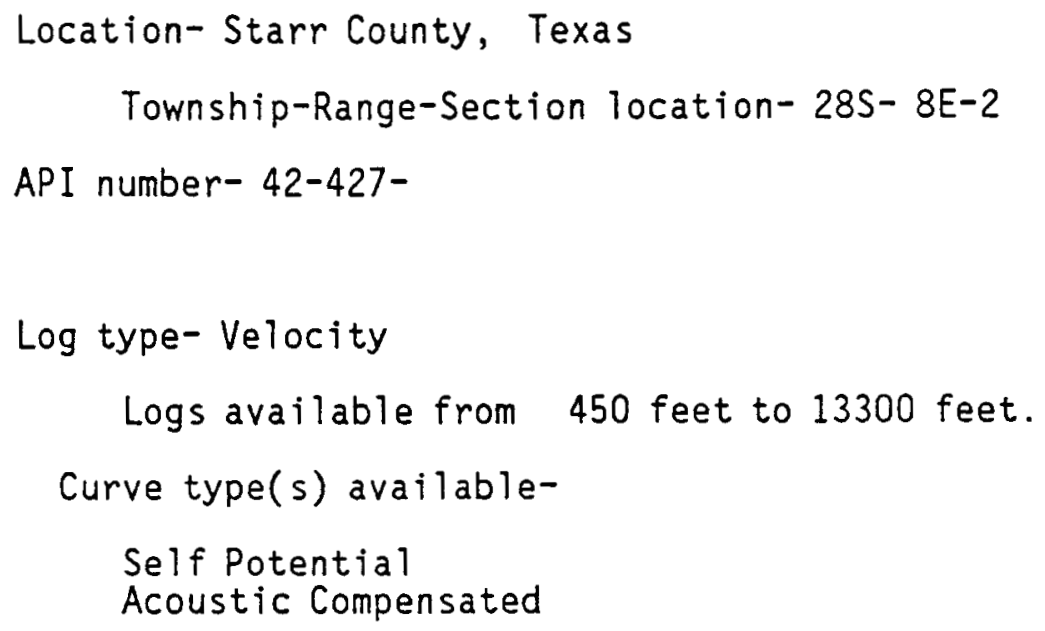

277. LOGDEX unique identification number- 356

Operator/Company- HUMBLE

We 11 name-S. EAST 22

Location- Kenedy County, Texas

Township-Range-Section location-25S-18E-3

API number- 42-261-

Log type-Velocity

Logs available from 100 feet to 17000 feet.

Curve type(s) available-

Self Potential

Acoustic Compensated

278. LOGDEX unique identification number- 357

Operator/Company- STANDARD OIL COMPANY OF TEXAS

Well name- RIO FARMS INC. 1

Location-Hidalgo County, Texas

Township-Range-Section location-30S-17E-2 
API number- 42-215-

Log type- Velocity

Logs available from 2600 feet to 17000 feet.

Curve type(s) available-

Self Potential

Acoustic Compensated

279. LOGDEX unique identification number- 358

Operator/Company- COASTAL STATES AND PRESTON OIL

We 11 name- E. DELCAMBRE 1

Location-Vermilion Parish, Louisiana

Township-Range-Section location- 14S- 5E-5

API number-17-113-

Log type- Velocity

Logs available from 12550 feet to 13020 feet.

Curve type(s) available-

Self Potential

Acoustic Compensated

Caliper

Log type- Gamma Ray

Logs available from 12700 feet to 12990 feet.

Curve type(s) available-

Gamma Ray API Units

Log type- Induction Electrical

Logs available from 2500 feet to 14510 feet.

Curve type(s) available-

Self Potential 


\section{Short Norma 1 \\ Induction Conductivity \\ Induction Resistivity}

Log type- Temperature

Logs available from 1000 feet to 13372 feet.

Curve type(s) available-

Temperature

Log type- Density

Logs available from 12400 feet to 12720 feet.

Curve type(s) available-

Density API Units

Porosity

Gamma Ray API Units

280. LOGDEX unique identification number- 359

Operator/Company- HUMBLE

Wel1 name- L. KUNETKA 1

Location-DeWitt County, Texas

Township-Range-Section location- 7S-24E-9

API number- 42-123-

Log type- Velocity

Logs available from 9980 feet to 12248 feet.

Curve type(s) available-

Self Potential

Acoustic Compensated

Caliper

281. LOGDEX unique identification number- 360

\section{Page 174}




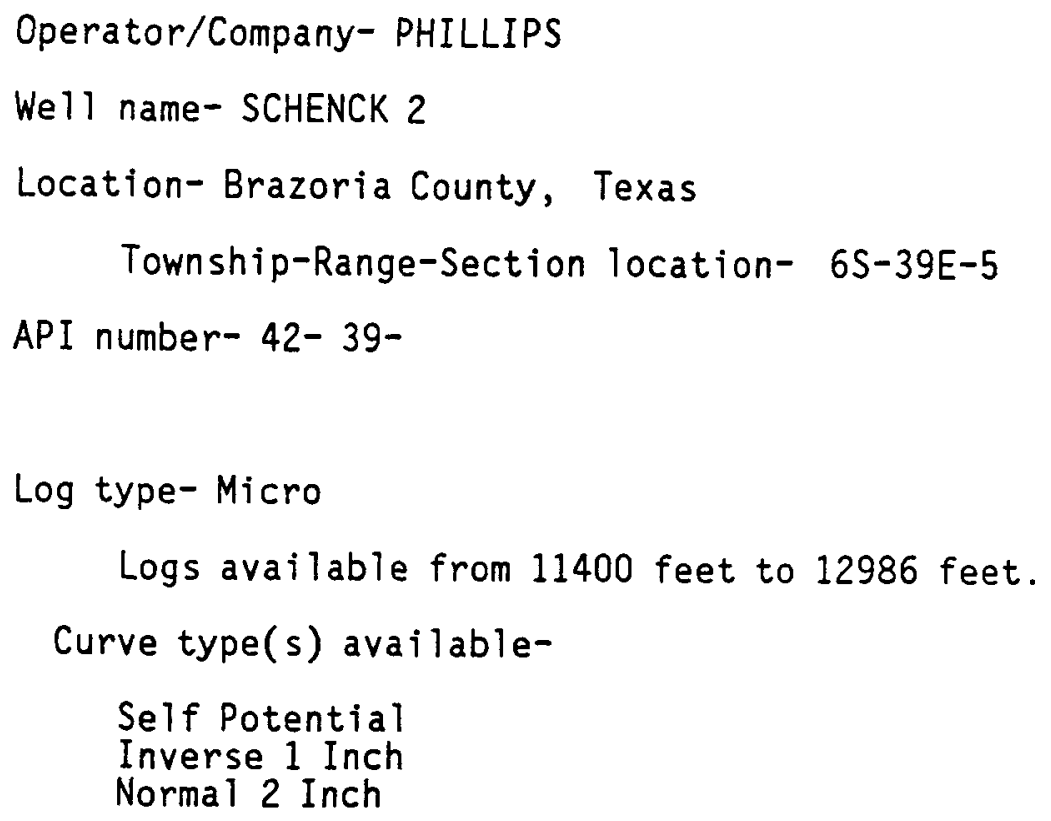

282. LOGDEX unique identification number- 361

Operator/Company- PHILLIPS

Well name- GEWIL 2

Location-Brazoria County, Texas

Township-Range-Section location- 6S-39E-5

API number- 42- 39-

Log type- Micro

Logs available from 8700 feet to 12250 feet.

Curve type(s) available-

Self Potential

Inverse 1 Inch

Normal 2 Inch

283. LOGDEX unique identification number- 362

Operator/Company - HANOVER 
Summary of LUGDEX Data Base

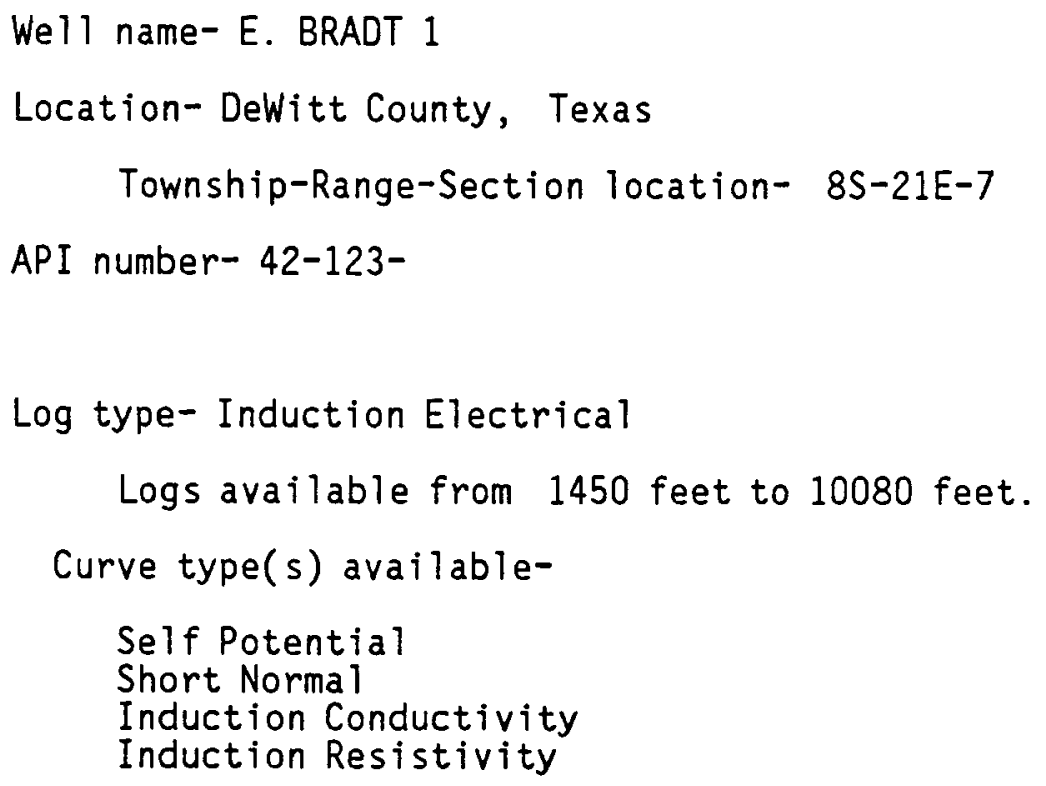

284. LOGDEX unique identification number- 363

Operator/Company- SHELL

We 11 name- $W$. OROEHL 1

Location- DeWitt County, Texas

Township-Range-Section location- 8S-21E-8

API number- 42-123-

Log type- Velocity

Logs available from 3550 feet to 14300 feet.

Curve type(s) available-

Self Potential

Acoustic Compensated

285. LOGDEX unique identification number- 364

Operator/Company- LONE STAR PRODUCING

We 11 name- H. GIPS 1 


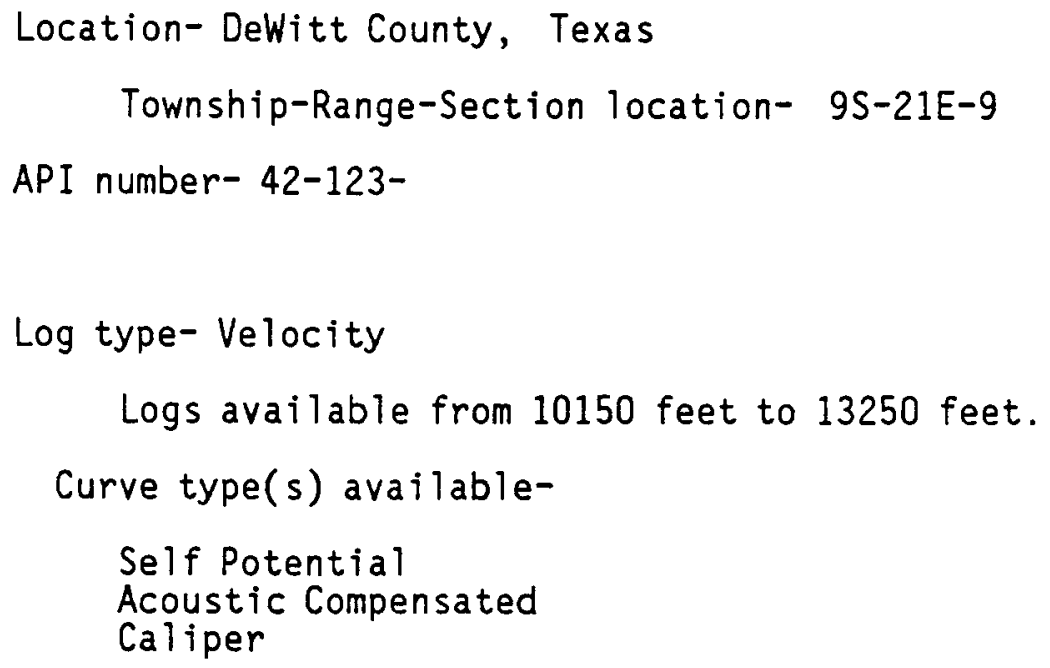

286. LOGDEX unique identification number- 365

Operator/Company-BRAZOS OIL AND GAS

Well name-T. BRYSCH 1

Location-DeWitt County, Texas

Township-Range-Section location- 9S-21E-6

API number- 42-123-

Log type-Velocity

Logs available from 1692 feet to 12020 feet.

Curve type(s) available-

Self Potential

Acoustic Compensated

Caliper

287. LOGDEX unique identification number- 366

Operator/Company- HUNT WM H-OCCIDENTAL

We 11 name- M. OSTERLOH 1

Location-DeWitt County, Texas 
Summary of LOGDEX Data Base

Township-Range-Section location- 9S-19E-6

API number- 42-123-

Log type- Velocity

Logs available from 3000 feet to 14900 feet.

Curve type(s) available-

Self Potential

Acoustic Compensated

Log type- Density

Logs available from 15800 feet to 16390 feet.

Curve type(s) available-

Density API Units

Caliper

Monitor

Log type- Porosity Neutron Compensated

Logs available from 14900 feet to 16390 feet.

Curve type(s) available-

Porosity

Caliper

288. LOGDEX unique identification number- 367

Operator/Company- HUMBLE

We 11 name- KESELING GAS UNIT NO 11

Location-DeWitt County, Texas

Township-Range-Section location- 8S-22E-7

API number- 42-123-

Log type- Velocity

Logs available from 6200 feet to 12024 feet. 
Curve type(s) available-

Self Potential

Acoustic Compensated

289. LOGDEX unique identification number- 368

Operator/Company- HARKINS AND HUMBLE

Wel1 name- KESELING GAS UNIT 21

Location- DeWitt County, Texas

Township-Range-Section location- 8S-22E-9

API number- 42-123-

Log type- Velocity

Logs available from 10394 feet to 11830 feet.

Curve type(s) available-

Self Potential

Acoustic Compensated

290. LOGDEX unique identification number- 369

Operator/Company-GEORGE R. BROWN

We11 name- A. FRIAR "A" 1

Location- DeWitt County, Texas

Township-Range-Section location- 8S-23E-2

API number- 42-123-

Log type- Velocity

Logs available from 1900 feet to 10400 feet.

Curve type(s) available-

Self Potential

Acoustic Compensated 
291. LOGDEX unique identification number- 370

Operator/Company- ATLANTIC

Well name- C. SCHORRE 1

Location- DeWitt County, Texas

Township-Range-Section Tocation- 8S-22E-9

API number- 42-123-

Log type- Velocity

Logs available from 6100 feet to 12000 feet.

Curve type(s) available-

Self Potential

Acoustic Compensated

292. LOGDEX unique identification number- 371

Operator/Company- BRIGHT AND SCHIFF

We 11 name- BROWN 1

Location-DeWitt County, Texas

Township-Range-Section location- 8S-23E-9

API number- 42-123-

Log type- Velocity

Logs available from 10700 feet to 12280 feet.

Curve type(s) available-

Self Potential

Acoustic Compensated

293. LOGDEX unique identification number- 372 
Operator/Company- HIGHLAND

Well name- J. WOOD JR. 1

Location- DeWitt County, Texas

Township-Range-Section location- $8 \mathrm{~S}-21 \mathrm{E}-8$

API number- 42-123-

Log type- Velocity

Logs available from 9400 feet to 14702 feet.

Curve type(s) available-

Self Potential

Acoustic Compensated

294. LOGDEX unique identification number- 373

Operator/Company- D.O.E. - GRUY FEDERAL, INC.

Well name- GRABBE 1

Location-Swisher County, Texas

Township-Range-Section location $=99 \mathrm{~N}-99 \mathrm{E}-9$

API number- 42-437-

Log type- Velocity

Logs available from 92 feet to 4200 feet.

Curve type(s) available-

Acoustic Compensated

Log type- Amplitude

Logs available from 90 feet to 4199 feet.

Curve type(s) available-

Acoustic Amp 1 itude

Log type- Porosity Neutron Compensated 
Logs available from 94 feet to 4207 feet.

Curve type(s) available-

Porosity

Ratio of Counters

Caliper

Gamma Ray API Units

Density API Units

Monitor

Log type- Induction Electrical

Logs available from 98 feet to 4189 feet.

Curve type(s) available-

Self Potential

Short Normal

Long Norma 1

Lateral

Induction Resistivity

Focussed Resistivity

295. LOGDEX unique identification number- 374

Operator/Company- D.O.E. - GRUY FEDERAL, INC.

We 11 name- R. WHITE 1

Location-Randa 11 County, Texas

Township-Range-Section location- 99N-99E-9

API number- 42-381-

Log type- Velocity

Logs available from 250 feet to 4000 feet.

Curve type(s) available-

Acoustic Compensated

Log type- Amplitude

Logs available from 100 feet to 4000 feet.

Curve type(s) available- 
Acoustic Amp litude

Log type- Porosity Neutron Compensated

Logs available from 96 feet to 4004 feet.

Curve type(s) available-

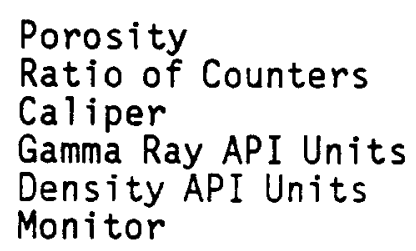

Log type- Induction Electrical

Logs available from 98 feet to 3988 feet.

Curve type(s) available-

Self Potential

Short Normal

Long Normal

Lateral

Induction Resistivity

Focussed Resistivity

296. LOGDEX unique identification number- 375

Operator/Company- MONSANTO AND PENNZOIL UNITED

Well name- DENTLER 1

Location- DeWitt County, Texas

Township-Range-Section location- 9S-21E-9

API number- 42-123-

Log type- Velocity

Logs available from 2700 feet to 9934 feet.

Curve type(s) available-

Self Potential

Acoustic Compensated 
297. LOGDEX unique identification number- 376

Operator/Company- AUSTRAL OIL

Well name- 0. SCHROETER 1

Location- DeWitt County, Texas

Township-Range-Section location- 9S-21E-1

API number- 42-123-

Log type- Velocity

Logs available from 2400 feet to 12000 feet.

Curve type(s) available-

Self Potential

Acoustic Compensated

Caliper

298. LOGDEX unique identification number- 377

Operator/Company- AUSTRAL OIL AND CROWN CENTRAL OIL

We 11 name- H. FERGUSON 1

Location- DeWitt County, Texas

Township-Range-Section location- 9S-22E-3

API number- 42-123-

Log type- Velocity

Logs available from 7200 feet to 12016 feet.

Curve type(s) available-

Self Potential

Acoustic Compensated

Caliper

Gamma Ray API Units 
299. LOGDEX unique identification number- 378

Operator/Company- HUMBLE

We11 name- GUARANTY TITLE \&TRUST 1

Location- DeWitt County, Texas

Township-Range-Section location- 9S-20E-7

API number- 42-123-

Log type- Velocity

Logs available from 1750 feet to 11800 feet.

Curve type(s) available-

Self Potential

Acoustic Compensated

Caliper

300. LOGDEX unique identification number- 379

Operator/Company- NEIL HANSON, EDWIN COX, MONSANTO, ET. AL.

We 11 name-MATEJEK 1

Location- DeWitt County, Texas

Township-Range-Section location- 9S-20E-8

API number- 42-123-

Log type- Velocity

Logs available from 3180 feet to 12000 feet.

Curve type(s) available-

Self Potential

Acoustic Compensated

Caliper 
301. LOGDEX unique identification number- 380

Operator/Company- EDWIN L. COX

Well name- OEHLKE 1

Location- DeWitt County, Texas

Township-Range-Section location- 9S-20E-8

API number- 42-123-

Log type- Induction Electrical

Logs available from 1900 feet to 11600 feet.

Curve type(s) available-

Self Potential

Induction Conductivity

Focussed Resistivity

Acoustic Compensated

302. LOGDEX unique identification number- 381

Operator/Company- SUPERIOR

Well name- H. BRANDT ET AL 1

Location-DeWitt County, Texas

Township-Range-Section location- 9S-21E-1

API number- 42-123-

Log type- Porosity Neutron Compensated

Logs available from 4500 feet to 13004 feet.

Curve type(s) available-

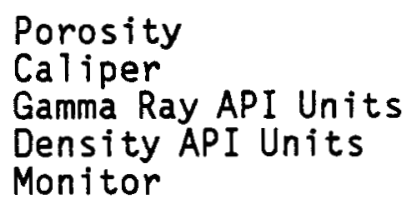


303. LOGDEX unique identification number- 382

Operator/Company- GETTY

Well name- NORDHEIM GAS UNIT 1515

Location- DeWitt County, Texas

Township-Range-Section location- 9S-20E-9

API number- 42-123-

Log type- Porosity Neutron Compensated

Logs available from 10000 feet to 11200 feet.

Curve type(s) available-

Porosity

Caliper

Gamma Ray API Units

Density API Units

Monitor

304. LOGDEX unique identification number- 383

Operator/Company- NEUHOFF

We 11 name- FAIRFAX FOSTER SUTTER 2

Location-Saint Mary Parish, Louisiana

Township-Range-Section location- 14S-10E-6

API number-17-101-

Log type- Induction Electrical

Logs available from 14400 feet to 16350 feet.

Curve type(s) available-

Self Potential

Induction Conductivity

Induction Resistivity

Focussed Resistivity 
305. LOGDEX unique identification number- 384

Operator/Company- SOUTHPORT EXPLORATION

Well name- B. SIMON 2

Location-Vermilion Parish, Louisiana

Township-Range-Section location- 11S-2E-6

API number- 17-113-

Log type- Induction Electrical

Logs available from 13950 feet to 15154 feet.

Curve type(s) available-

Self Potential

Induction Resistivity

Focussed Resistivity

306. LOGDEX unique identification number- 385

Operator/Company- EXXON

We 11 name- EXXON FEE 80

Location-Vermilion Parish, Louisiana

Township-Range-Section location-17S- 1E-5

API number- 17-113-

Log type- Velocity

Logs available from 13290 feet to 15700 feet.

Curve type(s) available-

Acoustic Compensated

Caliper

Gamma Ray API Units

Log type- Induction Electrical

Logs available from 3500 feet to 15700 feet.

Curve type(s) available-

Page 188 


\author{
Self Potential \\ Short Normal \\ Induction Resistivity \\ Gamma Ray API Units
}

307. LOGDEX unique identification number- 386

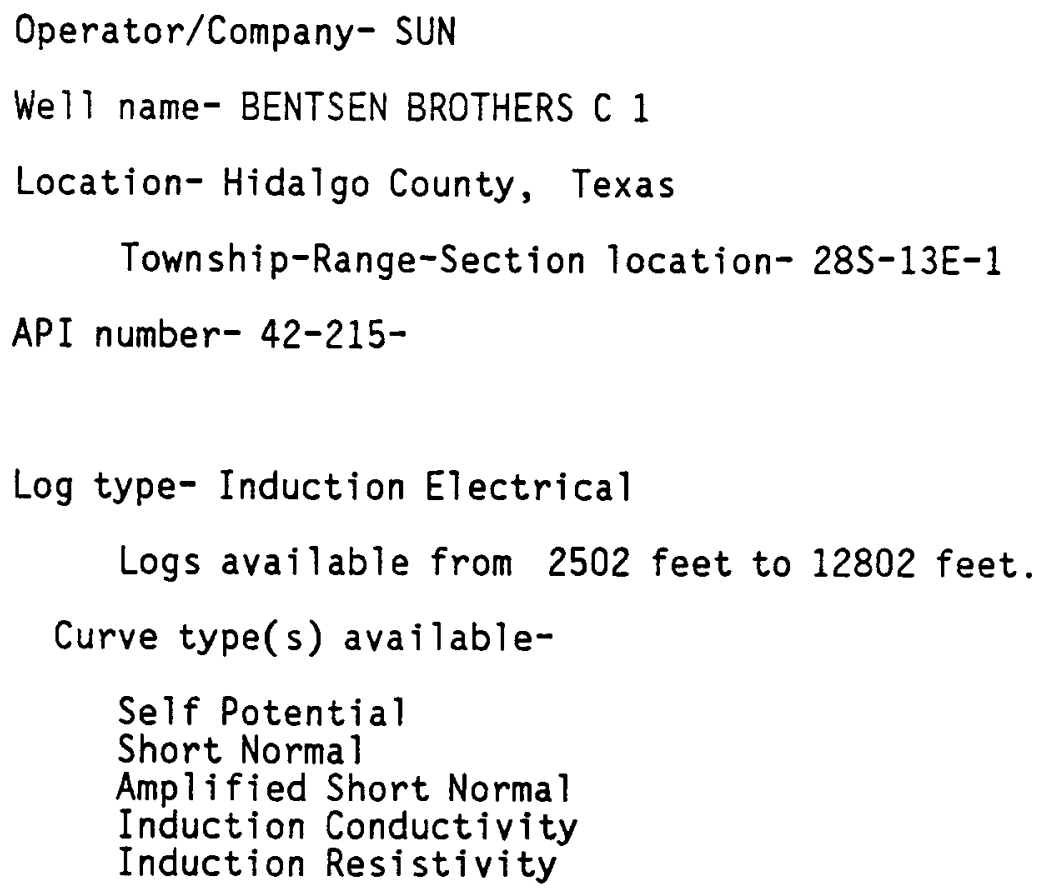

308. LOGDEX unique identification number- 387

Operator/Company- HUMBLE

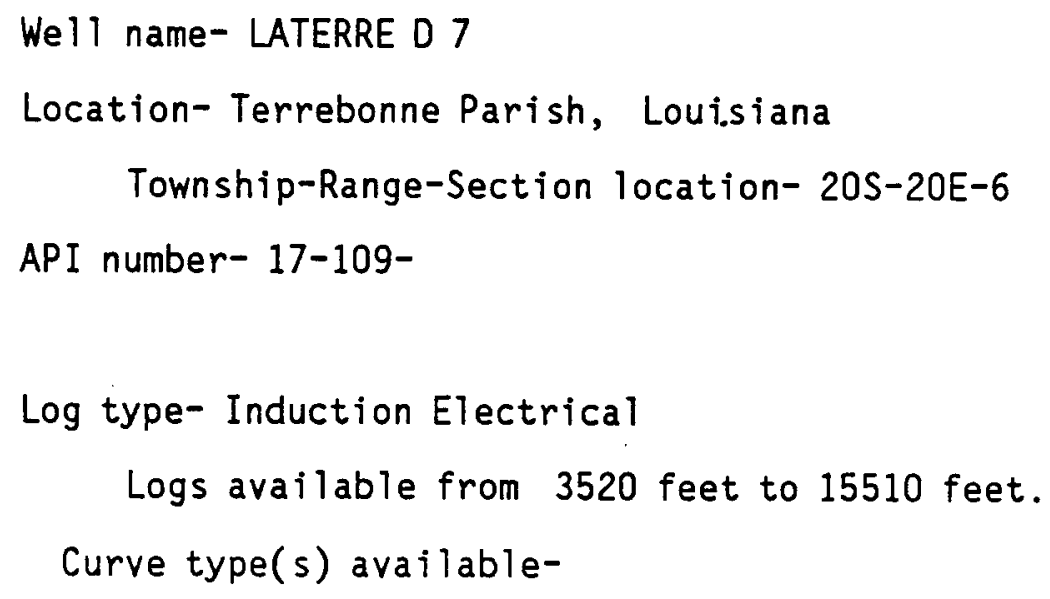


Self Potential

Short Normal

Amplified Short Normal

Induction Conductivity

Induction Resistivity

309. LOGDEX unique identification number- 388

Operator/Company- EXXON

We 11 name- C. M. ARMSTRONG 46

Location-Kenedy County, Texas

Township-Range-Section location-25S-18E-1

API number- 42-261-

Log type- Induction Electrical

Logs available from 15450 feet to 16216 feet.

Curve type(s) available-

Self Potential

Induction Conductivity

Induction Resistivity

Amplified Focussed Resistivity

310. LOGDEX unique identification number- 389

Operator/Company- TEXACO

Well name- H. H. THOMAS 16

Location-Matagorda County, Texas

Township-Range-Section location- 10S-31E-4

API number- 42-321-

Log type- Induction Electrical

Logs available from 1550 feet to 15000 feet.

Curve type(s) available- 
Self Potential

Induction Conductivity

Induction Resistivity

Focussed Resistivity

Acoustic Compensated

Log type- Porosity Neutron Compensated

Logs available from 6960 feet to 15000 feet.

Curve type(s) available-

Porosity

Caliper

Gamma Ray API Units

Density API Units

311. LOGDEX unique identification number- 390

Operator/Company- SUPERIOR

Well name- BURKE-HERMAN 1

Location-Matagorda County, Texas

Township-Range-Section location- 10S-31E-3

API number- 42-321-

Log type- Induction Electrical

Logs available from 1520 feet to 11830 feet.

Curve type(s) available-

Self Potential

Short Normal

Ampl ified Short Normal

Induction Conductivity

Induction Resistivity

312. LOGDEX unique identification number- 391

Operator/Company- SUPERIOR

We 11 name-W. NELSEN 1 
Summary of LOGDEX Data Base

Location-Matagorda County, Texas

Township-Range-Section location- 10S-31E-5

API number- 42-321-

Log type- Induction Electrical

Logs available from 1700 feet to 11730 feet.

Curve type(s) available-

Self Potential
Induction Conductivity
Induction Resistivity

313. LOGDEX unique identification number- 392

Operator/Company- COASTAL ŚTATES

We 11 name- WYLIE 1

Location- Matagorda County, Texas

Township-Range-Section location-10S-31E-2

API number- 42-321-

Log type- Induction Electrical

Logs available from 1270 feet to 13110 feet.

Curve type(s) available-

Self Potential

Induction Conductivity

Induction Resistivity

Focussed Resistivity

Amplified Focussed Resistivity

Log type- Velocity

Logs available from 1270 feet to 13114 feet.

Curve type(s) available-

Self Potential

Acoustic Non-Compensated

Acoustic Compensated

Page 192 
314. LOGDEX unique identification number- 393

Operator/Company- SUN

Well name- HEFFELFINGER 1

Location-Matagorda County, Texas

Township-Range-Section location-10S-31E-1

API number- 42-321-

Log type- Induction Electrical

Logs available from 2050 feet to 12002 feet.

Curve type(s) available-

Self Potential

Short Normal

Induction Conductivity

Induction Resistivity

315. LOGDEX unique identification number- 394

Operator/Company- HUMBLE

We 11 name- J. T. WILLIAMS 1

Location-Matagorda County, Texas

Township-Range-Section location- 10S-31E-1

API number- 42-321-

Log type- Induction Electrical

Logs available from 2066 feet to 11376 feet.

Curve type(s) available-

Self Potential

Short Normal

Amplified Short Normal

Induction Conductivity

Induction Resistivity 
316. LOGDEX unique identification number- 395

Operator/Company- MONSANTO CHEMICAL

Well name- BUCKEYE 1

Location- Matagorda County, Texas

Township-Range-Section location-10S-32E-3

API number- 42-321-

Log type- Induction Electrical

Logs available from 2506 feet to 16924 feet.

Curve type(s) available-

Self Potential

Short Norma 1

Ampl ified Short Normal

Long Normal

Latera 1

Induction Conductivity

Induction Resistivity

317. LOGDEX unique identification number- 396

Operator/Company-MONSANTO CHEMICAL

We 11 name- DOMAN 1

Location-Matagorda County, Texas

Township-Range-Section location- 10S-32E-3

API number- 42-321-

Log type- Induction Electrical

Logs available from 2140 feet to 12536 feet.

Curve type(s) available-

Self Potential

Short Normal

Long Norma 1 
Lateral

Induction Conductivity

Induction Resistivity

318. LOGDEX unique identification number- 397

Operator/Company- HUMBLE

Well name-PIERCE ESTATE 2

Location-Matagorda County, Texas

Township-Range-Section location-10S-32E-3

API number- 42-321-

Log type- Induction Electrical

Logs available from 2100 feet to 11700 feet.

Curve type(s) available-

Self Potential

Short Norma ]

Induction Conductivity

Induction Resistivity

319. LOGDEX unique identification number- 398

Operator/Company- COASTAL STATES

We 11 name- CORNELIUS 2

Location-Matagorda County, Texas

Township-Range-Section location- 9S-31E-8

API number- 42-321-

Log type- Induction Electrical

Logs available from 1262 feet to 13442 feet.

Curve type(s) available-

Self Potential 


\title{
Induction Conductivity \\ Induction Resistivity \\ Focussed Resistivity \\ Caliper
}

Log type- Velocity

Logs available from 1270 feet to 13470 feet.

Curve type(s) available-

\author{
Self Potential \\ Acoustic Non-Compensated \\ Acoustic Compensated
}

320. LOGDEX unique identification number-

Operator/Company- MONSANTO CHEMICAL

We 11 name- BEHRENS 1

Location-Matagorda County, Texas

Township-Range-Section location- 9S-32E-9

API number- 42-321-

Log type- Induction Electrical

Logs available from 2994 feet to 12512 feet.

Curve type(s) available-

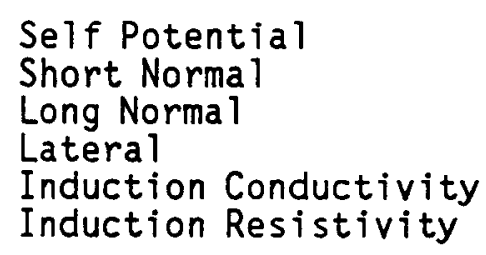

321. LOGDEX unique identification number- 400

Operator/Company- UNION PRODUCING

We 11 name- POTTMAST 1

Location-Matagorda County, Texas 
Township-Range-Section location-10S-30E-6

API number- 42-321-

Log type-Electrical

Logs available from 100 feet to 12300 feet.

Curve type(s) available-

Self Potential

Short Norma 1

Long Normal

Lateral

322. LOGDEX unique identification number- 401

Operator/Company- HUMBLE

Well name- J. C. LEWIS B 1

Location-Matagorda County, Texas

Township-Range-Section location- 9S-32E-8

API number- 42-321-

Log type- Induction Electrical

Logs available from 2050 feet to 12050 feet.

Curve type(s) available-

Self Potential

Short Normal

Induction Conductivity

Induction Resistivity

323. LOGDEX unique identification number- 402

Operator/Company-MONSANTO CHEMICAL

We11 name- MUNGER 1

Location-Matagorda County, Texas 


\author{
Township-Range-Section location- 9S-32E-9 \\ API number- 42-321- \\ Log type- Induction Electrical \\ Logs available from 1930 feet to 12530 feet. \\ Curve type(s) available- \\ Self Potential \\ Short Normal \\ Induction Conductivity \\ Induction Resistivity
}

324. LOGDEX unique identification number- 403

Operator/Company- CONTINENTAL

We 11 name-W. W. FONOREN ET AL 1

Location- Matagorda County, Texas

Township-Range-Section location- 9S-32E-7

API number- 42-321-

Log type- Induction Electrical

Logs available from 1300 feet to 13550 feet.

Curve type(s) available-

Self Potential

Short Normal

Induction Conductivity

Induction Resistivity

Gamma Ray API Units

325. LOGDEX unique identification number- 404

Operator/Company- TEXKAN

We 11 name- T. E. SLONE 1

Location- Matagorda County, Texas 
Township-Range-Section location- 10S-30E-7

API number- 42-321-

Log type- Induction Electrical

Logs available from 1300 feet to 12004 feet.

Curve type(s) available-

Self Potential

Short Normal

Induction Conductivity

Induction Resistivity

Gamma Ray API Units

326. LOGDEX unique identification number- 405

Operator/Company- TENNECO

We 11 name- B. W. TRULL ESTATE A-3

Location-Matagorda County, Texas

Township-Range-Section location-10S-30E-6

API number- 42-321-

Log type- Induction Electrical

Logs available from 1150 feet to 12500 feet.

Curve type(s) available-

Self Potential

Short Normal

Induction Conductivity

Induction Resistivity

Gamma Ray API Units

327. LOGDEX unique identification number- 406

Operator/Company- TENNECO

We 11 name- B. W. TRULL ESTATE A-2 
Summary of LOGDEX Data Base

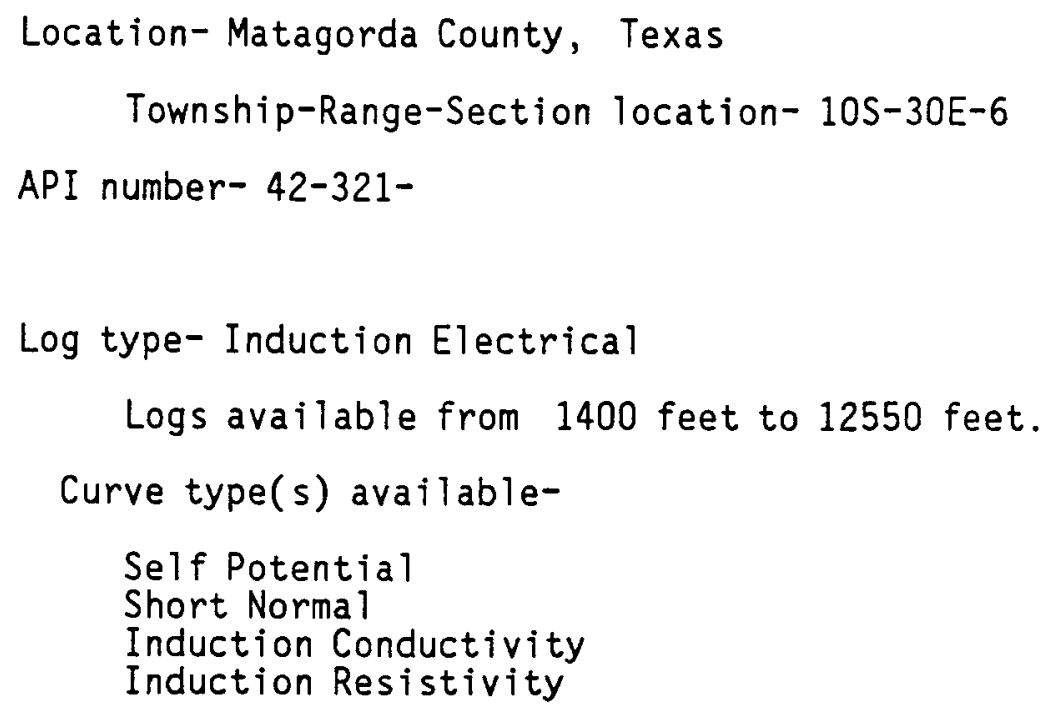

328. LOGDEX unique identification number- 407

Operator/Company- HUMBLE

Well name- C. M. ARMSTRONG 20

Location- Kenedy County, Texas

Township-Range-Section location- 25S-18E-7

API number- 42-261-

Log type- Induction Electrical

Logs available from 100 feet to 19280 feet.

Curve type(s) available-

Self Potential

Short Norma 1

Lateral

Induction Conductivity

Induction Resistivity

329. LOGDEX unique identification number- 408

Operator/Company- TEXACO

We 11 name- WEITING 15

Page 200 
Location-Brazoria County, Texas

Township-Range-Section location- $6 \mathrm{~S}-39 \mathrm{E}-8$

API number- 42- 39-

Log type- Electrical

Logs available from 416 feet to 12386 feet.

Curve type(s) available-

Self Potential

Short Norma 1

Long Norma 1

Lateral

330. LOGDEX unique identification number- 409

Operator/Company- SUN

We11 name- STATE-LAGUNA MADRE 1

Location- Kenedy County, Texas

Township-Range-Section location- 23S-21E-1

API number- 42-261-

Log type- Electrical

Logs available from 284 feet to 11138 feet.

Curve type(s) available-

Self Potential

Short Normal

Long Normal

Lateral

331. LOGDEX unique identification number- 410

Operator/Company- SUN

We 11 name- JEFFERIES 2 
Summary of LUGDEX Data Base

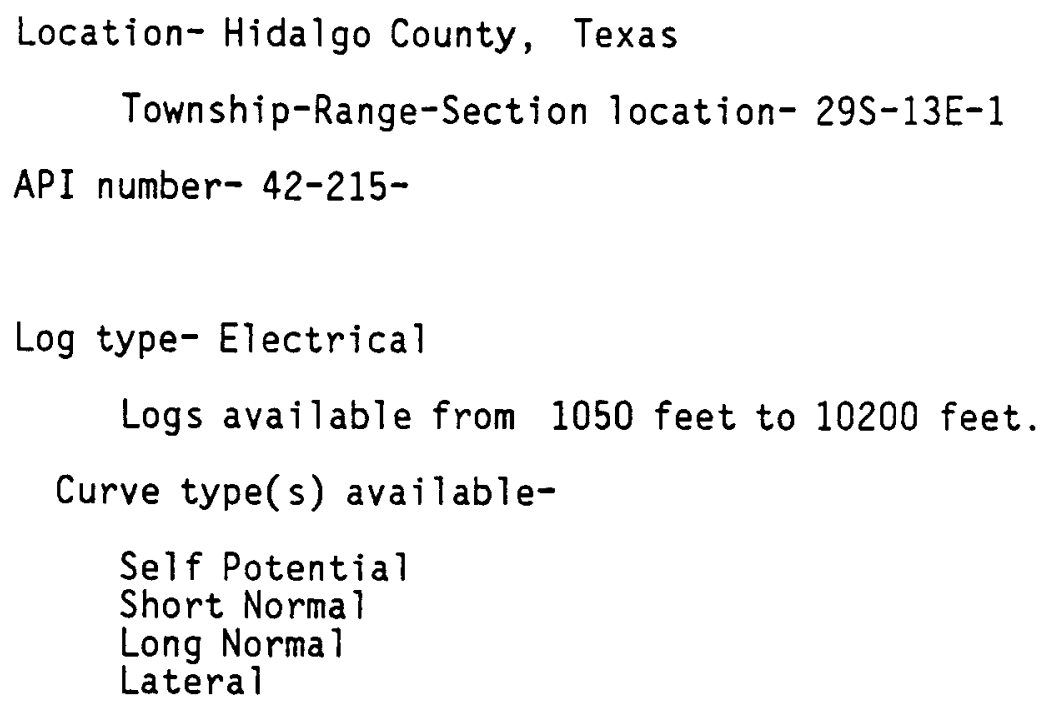

332. LOGDEX unique identification number- 411

Operator/Company- C \& K PETROLEUM

We 11 name-POTTHAST 1

Location-Matagorda County, Texas

Township-Range-Section location-10S-30E-6

API number- 42-321-

Log type- Induction Electrical

Logs available from 2500 feet to 12334 feet.

Curve type(s) available-

\section{Self Potential}

Induction Conductivity

Induction Resistivity

Focussed Resistivity

333. LOGDEX unique identification number- 412

Operator/Company- SUN

Well name- 768 


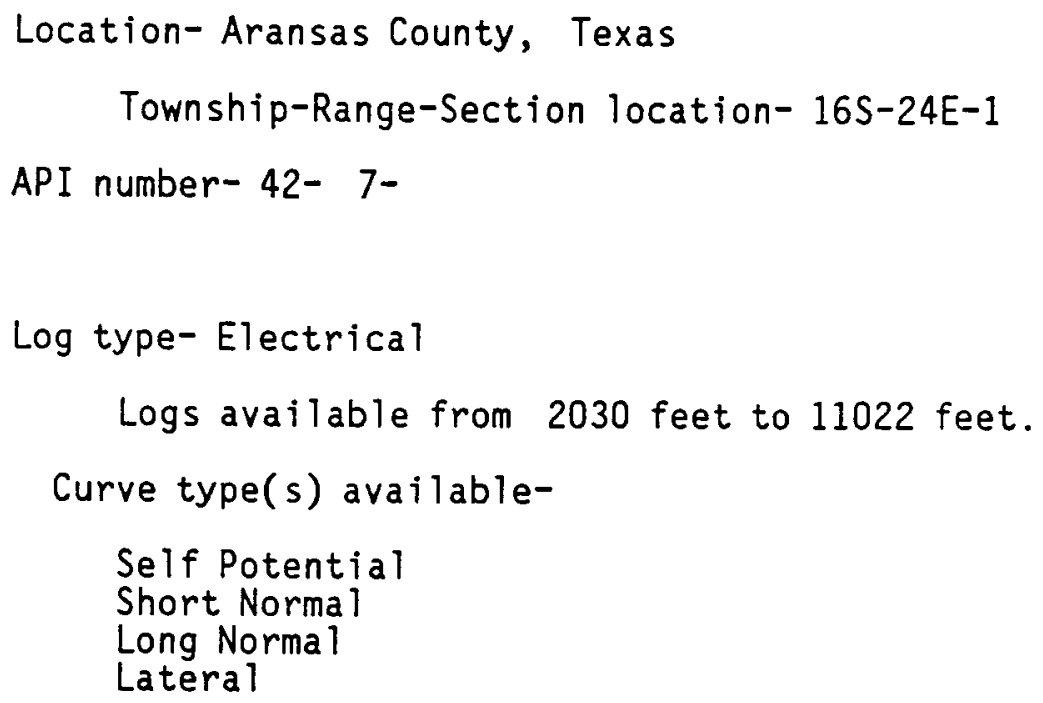

334. LOGDEX unique identification number- 413

Operator/Company- PHILLIPS

We 11 name- HOUSTON Z 1

Location-Brazoria County, Texas

Township-Range-Section location- 6S-39E-8

API number- 42- 39-

Log type- Electrical

Logs available from 200 feet to 14290 feet.

Curve type(s) available-

Self Potential

Short Normal

Long Norma 1

Lateral

335. LOGDEX unique identification number- 414

Operator/Company- PAN AMERICAN

We11 name- SHERRILL GAS UNIT 1 
Summary of LOGDEX Data Base

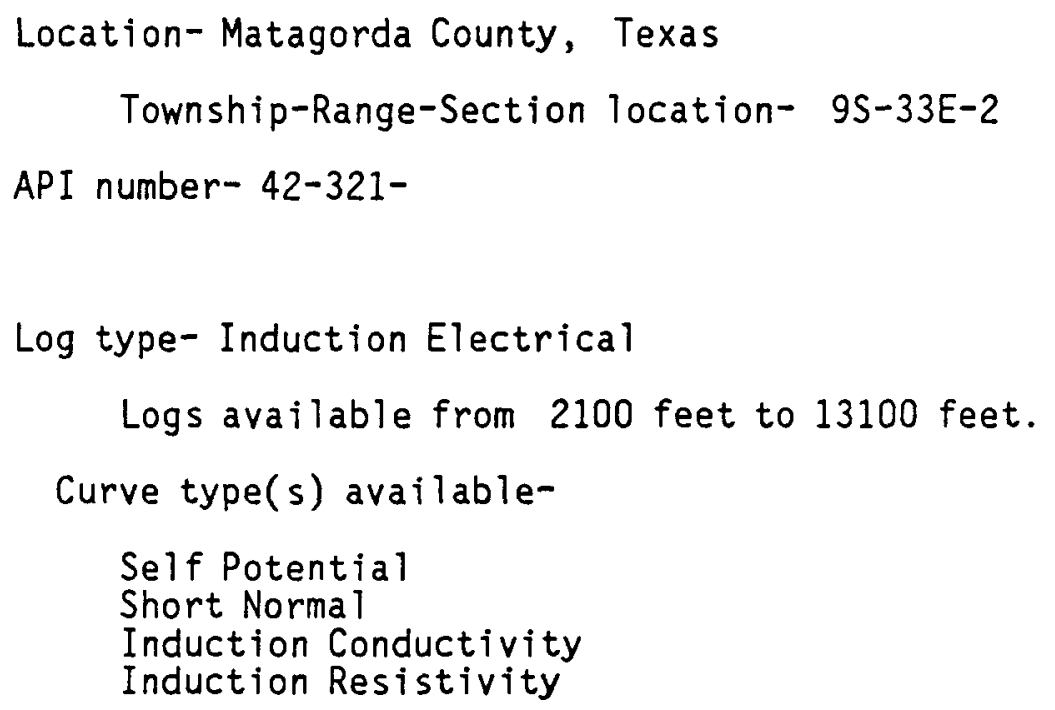

336. LOGDEX unique identification number- 415

Operator/Company- HUMBLE

We 11 name- P. HUEBNER 1

Location-Matagorda County, Texas

Township-Range-Section location- 9S-33E-6

API number- 42-321-

Log type- Induction Electrical

Logs available from 100 feet to 14000 feet.

Curve type(s) available-

Self Potential

Short Normal

Lateral

Induction Conductivity

Induction Resistivity

337. LOGDEX unique identification number- 416

Operator/Company- GULF

We11 name- GILMORE ET AL 1 


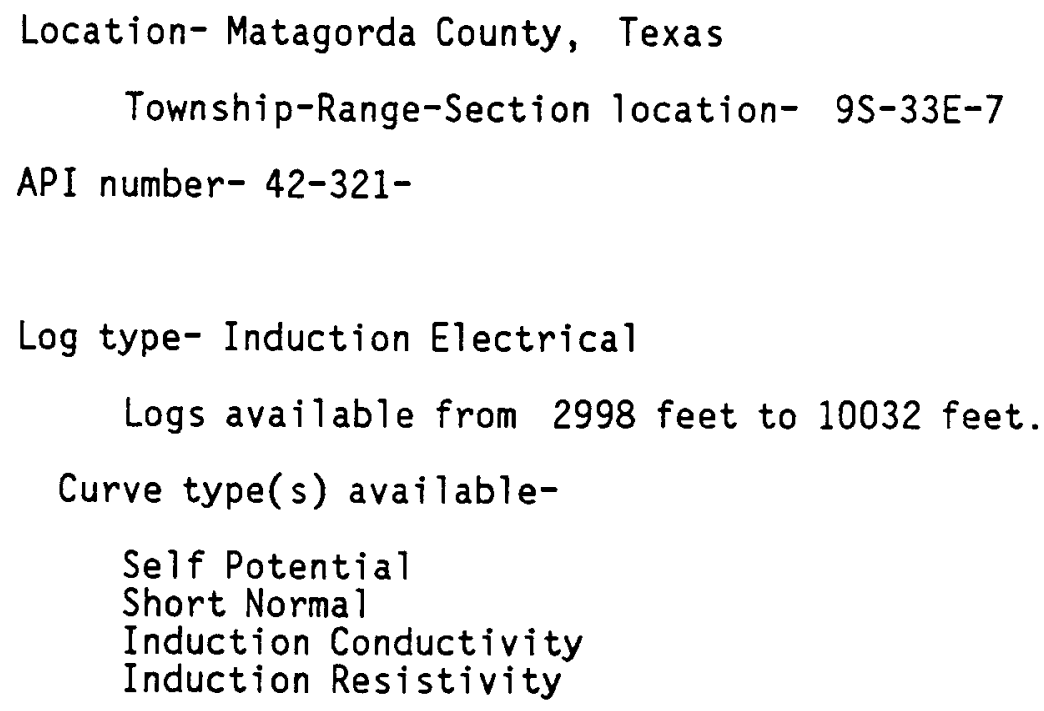

338. LOGDEX unique identification number- 417

Operator/Company- CYPRUS

We 11 name- RUGLEY 1

Location- Matagorda County, Texas

Township-Range-Section location- 8S-33E-9

API number- 42-321-

Log type- Induction Electrical

Logs available from 2400 feet to 12602 feet.

Curve type(s) available-

Self Potential

Short Normal

Induction Conductivity

Induction Resistivity

339. LOGDEX unique identification number- 418

Operator/Company- BRADCO

We 11 name- E. BURKHART ET AL 1 
Summary of LOGDEX Data Base

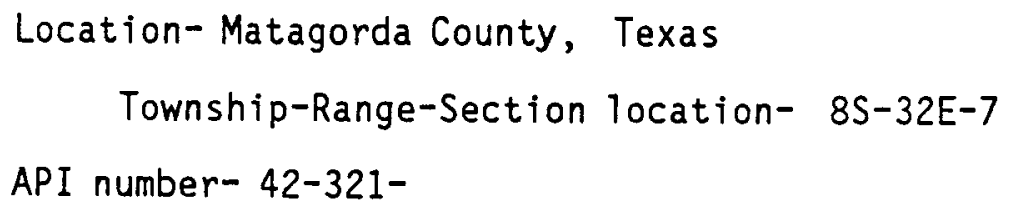

340. LOGDEX unique identification number- 419

Operator/Company-MICHAEL T. HALBOUTY

Well name- M. E. CROUCH 1

Location-Matagorda County, Texas

Township-Range-Section location- 8S-32E-5

API number- 42-321-

Log type- Induction Electrical

Logs available from 1500 feet to 8800 feet.

Curve type(s) available-

Self Potential

Short Normal

Induction Conductivity

Induction Resistivity

341. LOGDEX unique identification number- 420

Operator/Company- TEXACO

We 11 name- PIERCE ESTATES 143 


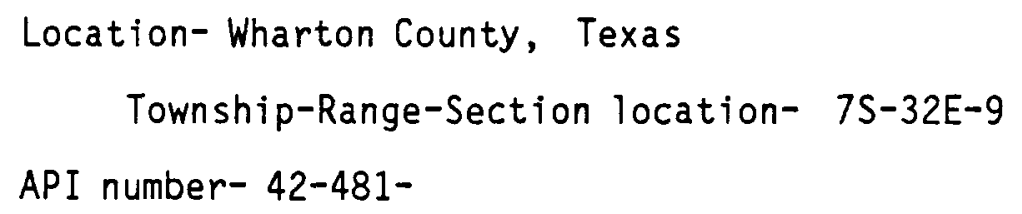

342. LOGDEX unique identification number- 421

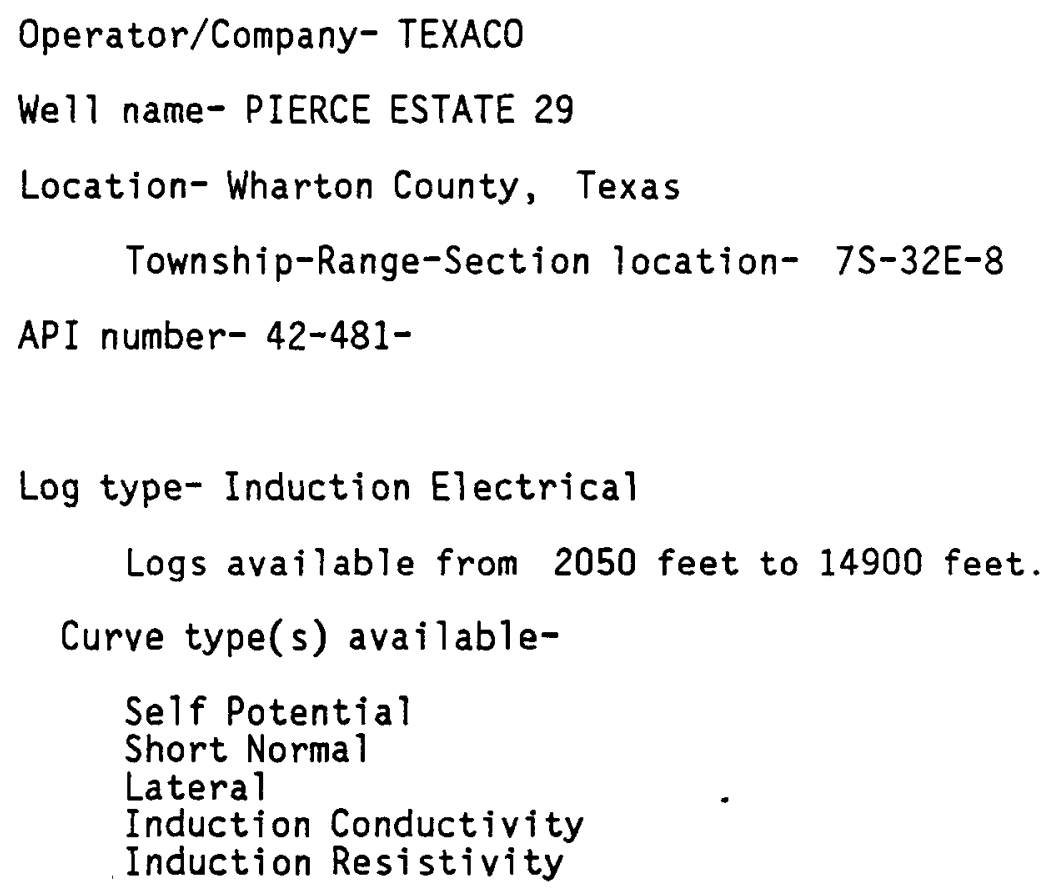

343. LOGDEX unique identification number- 422

Operator/Company-MOBIL

We11 name- E. CORNELIUS 15 
Summary of LOGDEX Data Base

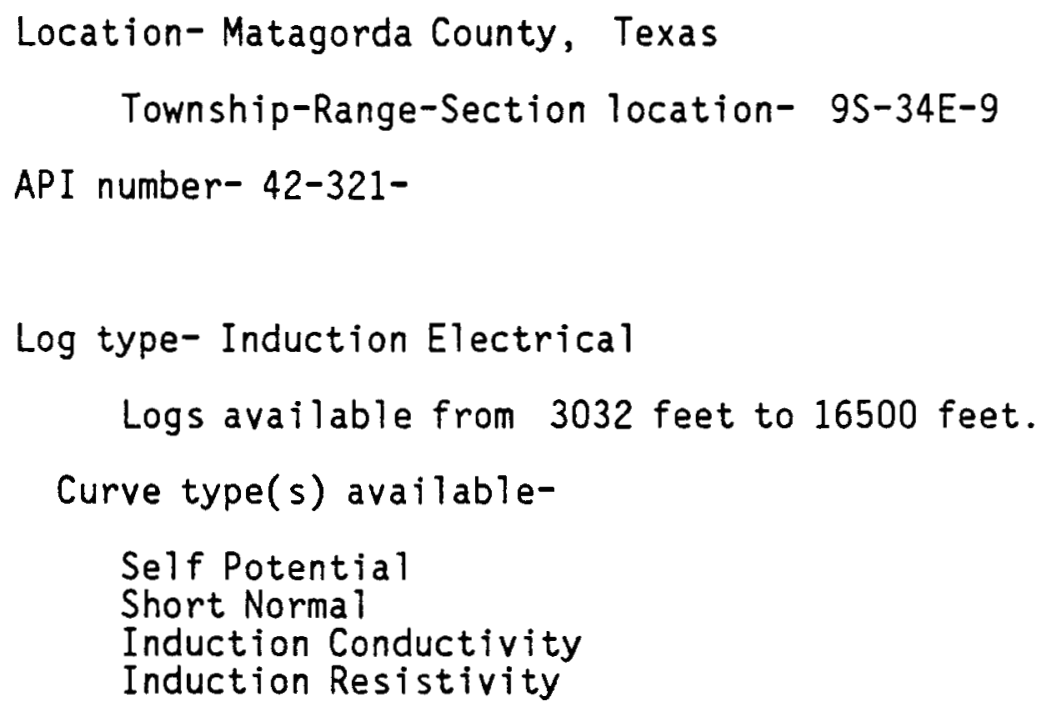

344. LOGDEX unique identification number- 423

Operator/Company- SKELLY

We 11 name- GEST 1

Location- Matagorda County, Texas

Township-Range-Section location- 9S-33E-2

API number- 42-321-

Log type- Electrical

Logs available from 2050 feet to 12000 feet.

Curve type(s) available-

Self Potential

Short Normal

Long Normal

Lateral

345. LOGDEX unique identification number- 424

Operator/Company- MAGNOLIA

Well name- LE TULLE 1 


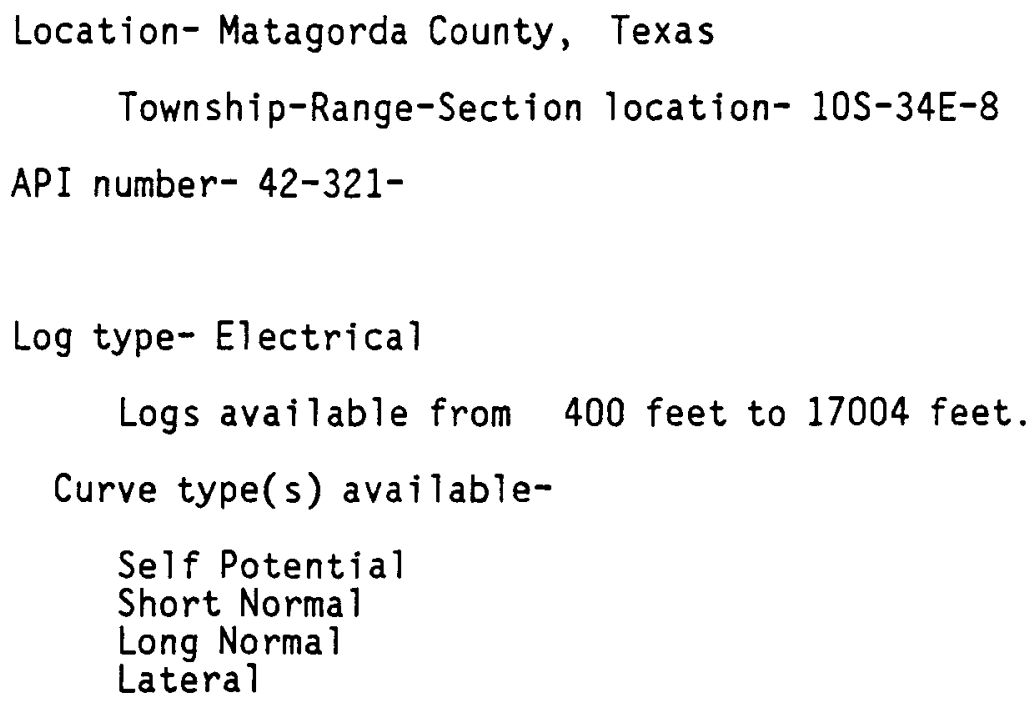

346. LOGDEX unique identification number- 425

Operator/Company- WAINOCO

Well name- $P$. R. LIROUARD 1

Location- Lafayette Parish, Louisiana

Township-Range-Section location- 11S- 5E-1

API number- 17- 55-

Log type- Induction Electrical

Logs available from 12650 feet to 14896 feet.

Curve type(s) available-

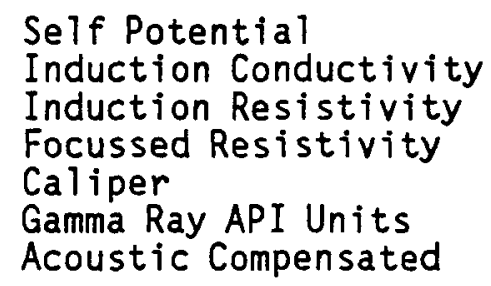

347. LOGDEX unique identification number- 426 Operator/Company- CITIES SERVICE \& SUNRAY 
Summary of LOGDEX Data Base

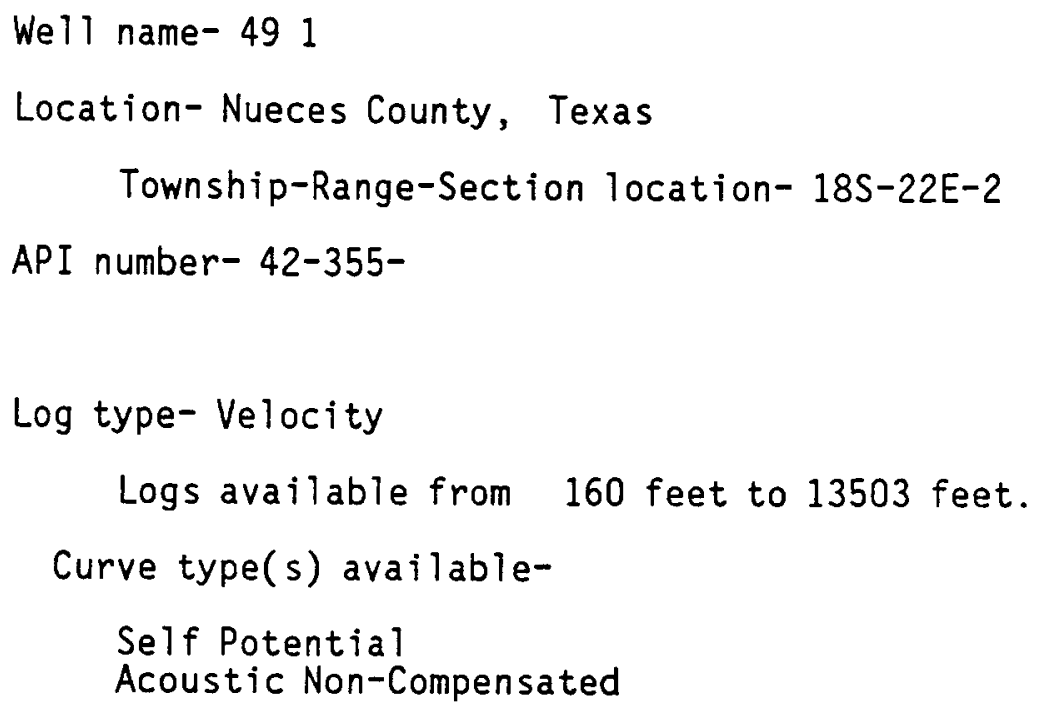

348. LOGDEX unique identification number- 427

Operator/Company- CITIES SERVICE

Well name- 721

Location- Nueces County, Texas

Township-Range-Section location- 18S-22E-2

API number- 42-355-

Log type- Velocity

Logs available from 1900 feet to 10452 feet.

Curve type(s) available-

Self Potential

Acoustic Non-Compensated

349. LOGDEX unique identification number- 428

Operator/Company-CITIES SERVICE

We 11 name- 841

Location- Nueces County, Texas 
Township-Range-Section location- 19S-22E-9

API number- 42-355-

Log type- Velocity

Logs available from 2520 feet to 10367 feet.

Curve type(s) available-

Self Potential

Acoustic Non-Compensated

350. LOGDEX unique identification number- 429

Operator/Company-CITIES SERVICE

Well name- 211

Location- Nueces County, Texas

Township-Range-Section location- 18S-22E-6

API number- 42-355-

Log type-Velocity

Logs available from 1560 feet to 14514 feet.

Curve type(s) available-

Self Potential

Acoustic Non-Compensated

351. LOGDEX unique identification number- 430

Operator/Company- CITIES SERVICE

Well name- LOGGAN "A" 1

Location- Nueces County, Texas

Township-Range-Section location- 20S-19E-3

API number- 42-355- 
Summary of LOGDEX Data Base

Log type- Velocity

Logs available from 1970 feet to 13106 feet.

Curve type(s) available-

Self Potential

Acoustic Non-Compensated

352. LOGDEX unique identification number- 432

Operator/Company- E. COCKRELL, JR.

Well name- NEUSZER 1

Location- Matagorda County, Texas

Township-Range-Section location- 10S-30E-8

API number- 42-321-

Log type- Velocity

Logs available from 9760 feet to 16188 feet.

Curve type(s) available-

Self Potential

Acoustic Non-Compensated

353. LOGDEX unique identification number- 434

Operator/Company- CONTINENTAL

Well name-W. W. FONDREN, JR., ET AL 1

Location-Matagorda County, Texas

Township-Range-Section location- 9S-32E-7

API number- 42-321-

Log type- Velocity

Logs available from 7700 feet to 13512 feet. 
Curve type(s) available-

Self Potential

Acoustic Compensated

354. LOGDEX unique identification number- 435

Operator/Company- HUMBLE

Well name- J. C. LEWIS 1

Location-Matagorda County, Texas

Township-Range-Section location- 10S-32E-2

API number- 42-321-

Log type- Velocity

Logs available from 1970 feet to 11728 feet.

Curve type(s) available-

Self Potential

Acoustic Non-Compensated 
Summary of LOGDEX Data Base

Page 214 


\subsection{Appendix A}

Well Classification Codes

$\begin{array}{ll}\text { D\&A } & \text { Dry Hole } \\ \text { OIL } & \text { 0il Well } \\ \text { GAS } & \text { Gas Well } \\ \text { M-O } & \text { Multiple Completion Oil } \\ \text { M-G } & \text { Multiple Completion Gas } \\ \text { MOG } & \text { Multiple Completion Oil and Gas } \\ \text { J\&A } & \text { Junked and Abandoned } \\ \text { INJ } & \text { Injection Well } \\ \text { SER } & \text { Service Wells All Types } \\ \text { AOW } & \text { Abandoned 0il Well } \\ \text { AGW } & \text { Abandoned Gas Well } \\ \text { T\&A } & \text { Temporarily Abandoned } \\ \text { SUS } & \text { Suspended Operation }\end{array}$


Summary of LOGDEX Data Base

•

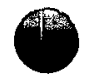

Page 216 
Summary of LOGDEX Data Base

Page 218 


\subsection{INDEX}

\section{c}

Classification

D\&A $6,13,14,17,20,21,22,23,26,28,29,33,36,37,38,43,44$, $45,47,48,49,50,51,52,53,54,55,56,57,62,63,65,66,67,68$, $70,73,74,75,76,78,80,81,82,83,84,85,86,87,89,91,93,94$, $95,97,99,100,101,102,103$

GAS $5,6,8,10,12,15,23,24,30,35,36,43,46,57,68,69,72,73$, $77,79,87,90,98,104,106,108,110,112,114,115,129,130,132$, $134,135,138,139,140,143,145,146,147,156,158$

J\&A $27,39,96$

$M-G \quad 71,81,159$

MOG 161

OIL $18,41,66$

T\&A 16

County or Parish

Aransas 156,160,165, 203

Aust in $124,141,143$

Bee 167

Brazoria $5,6,7,8,9,10,12,13,14,15,16,17,18,19,21,22,25$, $26,27,28,29,30,32,35,36,37,38,39,40,41,42,43,44,45,46$, $47,48,49,51,52,53,54,55,56,57,58,59,60,61,62,63,64,65$, $66,67,68,69,82,83,84,85,9$

Calhoun 159

Cameron 166, 167

Colorado $142,145,146$

DeWitt 102,106, 107, 108, 109, 110, 161, 174, 176, 177, 178, 179, 180, $181,183,184,185,186,187$

Duvai 129, 130,131,132,133,148,162,163,171

Fort Bend $61,79,80,81,82,126$

Galveston $11,20,23,24,31,33,34,35,41,60,70,71,72,73,74,75$, $76,77,78,86,87,88,89,90,91,92,93,94,95,97,98$

Goliad $103,105,112,168$

Grimes 116

Harris $115,117,118,121,122,126,127,138,139,140,168$

Hidalgo 9, 46, $172,189,202$

Jim Hogg 150, 155

Karnes 102, 111

Kenedy 99,100,101, 104,166,171, 172, 190, 200, 201

Lafayette 209

Lavaca 104,169

Liberty 151,169

Live Oak 133, 134, 135,136,137, 138

Matagorda 50, 166, 170, 190, 1931, 192, 193, 194, 195, 196, 197, 198, $199,200,202,204,205,206,208,209,212,213$

McMuilen $127,128,163$

Montgomery $113,114,115,116,118,119,143,144$

Nueces $157,159,165,210,211$

Randa 11182

Saint Mary 187

San Jacinto 152,153

San Patricio 157, 158, 170 
Starr 172

Swisher 181

Terrebonne 189

Vermilion 173,188

Victoria 105,111

Waller 113, 117, 122, 123, 125, 139

Webb $148,149,156,162,164$

Wharton 207

Wood 19

Zapata $147,154,155$

Curve types

Acoustic Amplitude 181,183

Acoustic Compensated $5,6,7,8,9,10,12,13,14,15,16,17,18,19$, $20,25,26,28,29,32,33,35,76,77,97,110,111,120,150,161,162$, $163,164,165,166,167,168,169,170,171,172,173,174,176,177$, $178,179,180,181,182,183,184$

Acoustic Non-Compensated $7,8,14,17,18,21,22,23,24,27,28,30$, $32,34,43,45,47,192,196,210,211,212,213$

Amplified Focussed Resistivity $20,21,25,28,29,32,33,34,52,58$, $59,60,74,97,98,190,192$

Amp i ified Short Normal $5,7,10,11,12,15,16,17,18,19,21,22,24$, $25,26,27,29,30,31,33,35,36,37,38,39,40,41,42,43,44,45$, $46,47,48,49,50,51,52,53,54,55,56,57,58,59,61,62,63,64$, $65,66,67,68,72,73,74,75$,

Caliper $5,6,7,8,9,10,11,12,13,14,15,16,17,18,19,20,21$, $22,23,24,26,27,28,29,30,31,32,34,35,43,45,47,70,71,72$, $76,77,96,110,120,121,161,163,173,174,177,178,182,183,184$, $185,186,187,188,191,196,209$

Density API Units $9,11,13,20,34,96,121,163,174,178,182,183$, $186,187,191$

Density Company Units 14

Focussed Resistivity $20,21,25,28,29,32,33,34,44,52,58,59,60$, $65,74,88,97,98,103,110,116,120,123,132,135,136,140,141$, $143,144,145,152,156,182,183,186,187,188,191,192,196,202$, 209

Gamma Ray API Units 9, 11, 20, 21, 26, 29, 30, 34,60,96, 110, 118, 120, $121,131,139,149,156,160,163,164,168,169,173,174,182,183$, $184,186,187,188,189,191,198,199,209$

Gamma Ray Company Units 31,120

Induction Conductivity $5,7,10,11,12,15,17,18,19,20,21,22,24$, $25,26,27,28,29,30,32,33,34,35,36,37,38,39,41,43,44,45$, $46,47,49,50,52,53,55,56,57,58,59,60,61,62,63,64,65,67$, $68,72,74,75,77,78,79,81$,

Induction Resistivity $5,7,10,11,12,15,16,17,18,19,20,21,22$, $24,25,26,27,28,29,30,32,33,34,35,36,37,38,39,41,43,44$, $45,46,47,49,50,52,53,55,56,57,58,60,61,62,63,64,65,67$, $68,72,74,76,77,78,79,81,8$

Inverse 1 Inch $27,31,69,70,71,72,175$

Lateral $25,31,40,42,43,44,46,48,51,53,54,55,59,61,63,64$, $65,66,67,69,73,74,75,80,83,84,85,86,89,90,91,92,93,95$, $96,99,126,128,138,151,162,164,182,183,194,195,196,197,200$, $201,202,203,204,207,208,20$

Long Norma $124,31,40,42,43,44,46,48,51,53,54,55,59,63,64$, $65,66,67,68,73,74,75,80,83,84,85,86,89,90,91,92,93,95$, $96,99,100,106,107,113,115,119,124,127,151,182,183,194,196$, $197,201,202,203,208,209$

Monitor $9,11,13,20,34,96,121,163,178,182,183,186,187$

Neutron API Units 26,156 
Neutron Company Units 31

Normal 2 Inch 175

Porosity $96,121,174,178,182,183,186,187,191$

Potassium 121

Ratio 121

Ratio of Counters 182,183

Self Potential $5,6,7,8,9,10,11,12,13,14,15,16,17,18,19,20$, $21,22,23,24,25,26,27,28,29,30,31,32,33,34,35,36,37,38$, $39,40,41,42,43,44,45,46,47,48,49,50,51,52,53,54,55,56$, $57,58,59,60,61,62,63,64$,

Short Normal $5,7,10,11,12,15,16,17,18,19,21,22,24,25,26$, $27,29,30,31,33,35,36,37,38,39,40,41,42,43,44,45,46,47$, $48,49,50,51,52,53,54,55,56,57,58,59,61,62,63,64,65,66$, $67,68,72,73,74,75,77,78,79$

Sigma 121

Temperature 120,174

Thorium 121

Uranium 121

\section{L}

Log types

Amp 1 itude 181,182

Decay 120

Density $8,10,13,14,20,34,163,174,178$

Dual Indcution 96,120

Electrical $31,40,41,42,48,51,53,54,59,63,64,65,66,67,68$, $73,74,75,80,83,84,85,89,90,91,92,95,98,100,106,107,113$, $115,119,124,127,197,201,202,203,208,209$

Gamma Ray 173

Gamma Ray Neutron 26, 31, 156

Induction Electrical $5,7,10,11,12,14,15,16,17,18,19,20,21$, $22,24,25,26,27,28,29,30,31,32,33,34,35,36,37,38,39,41$, $43,44,45,46,47,48,49,50,51,52,53,55,56,57,58,59,60,61$, $62,64,65,66,68,72,73,75,77$

Micro $27,30,69,70,71,72,175$

Porosity Neutron Compensated $96,121,178,181,183,186,187,191$

Spectralog 121

Temperature 120,174

Velocity $5,6,7,8,9,10,11,12,13,14,15,16,17,18,19,20,21$, $22,23,24,26,27,28,30,32,33,35,43,45,47,76,97,110,111$, $120,150,161,162,164,165,166,167,168,169,170,171,172,173$,

$174,176,177,178,179,180,181,18$

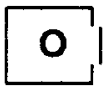

Operators

ADA 68

AMBASSADOR OIL CORPORATION 36

AMOCO 152, 170

ANSCHUTZ 57

ARNOLD O MORGAN 157

Page 221 


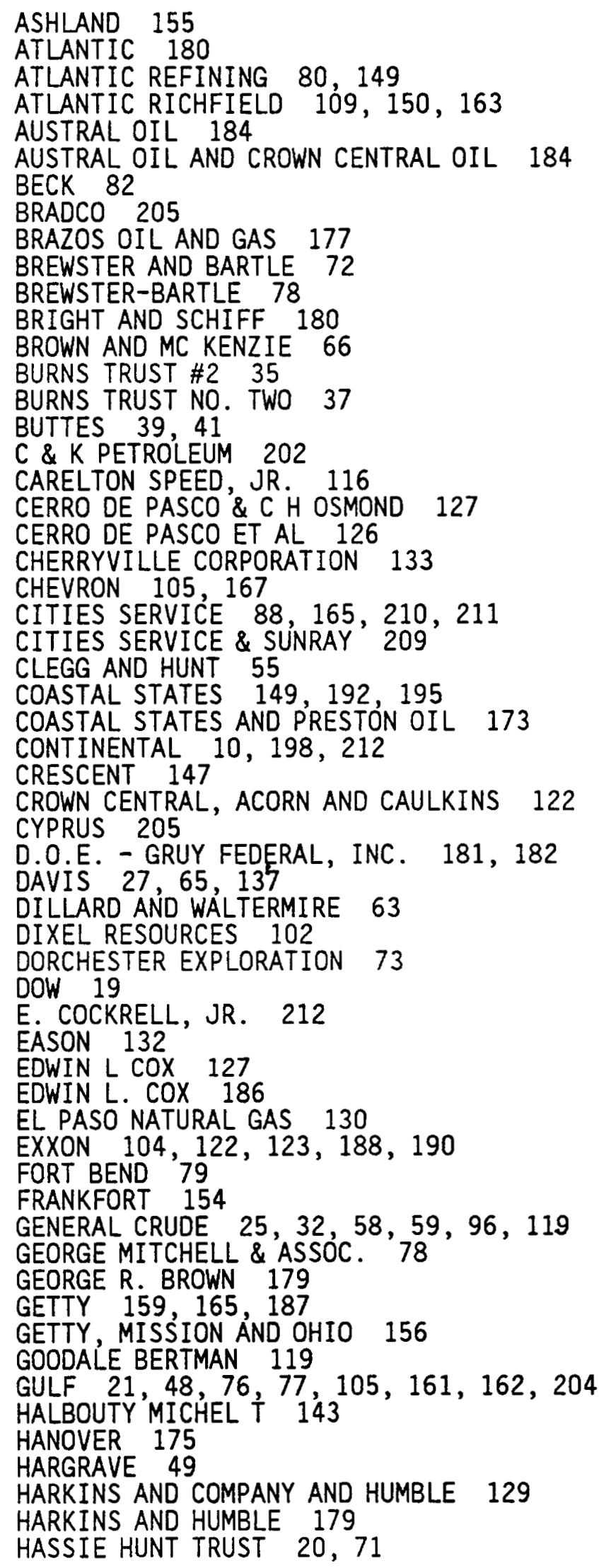

Page 222 
HIGHLAND 181

HOUSTON NATURAL GAS 139

HOUSTON OIL \& MINERALS 97,98

HOUSTON OIL AND MINERALS 29

HUGHES \& HUGHES 161

HUGO ALLEN 148

HUMBLE $7,9,12,19,22,28,29,34,42,51,74,92,98,99,100,101$, $117,118,131,134,135,139,157,171,172,174,178,185,189,193$, $195,197,200,204,213$

HUMBLE AND JACK FRAŻIER 83

HUMBLE OIL AND REFINING 112

HUNT WM H-OCCIDENTAL 177

INTEGRAL ENERGY 135

J P OWEN 125

J. K. DORRANCE 82

J. S. MICHAEL 53,85

JOCELYN-VARN, COLORADO, JADE 75

JOHN W. MECOM 72

JOHN W. MECON 89, 90

JOHN W. MECON ET AL 89

KILLAM \& HURD LTD. 164

KILROY , TESORO AND SUBURBAN 134

KING RESOURCES 142

KIRBY PET AND RUSSELL MC FARLAND 52

LAMAR HUNT 102

LARIO AND BELMONT 58

LONE STAR 45

LONE STAR PRODUCING 103, 109, 111, 176

M.P.S PRODUCTION -NORTH CENTRAL OIL -HARRY HURT 138

MAGNOLIA 107,208

MANA OIL \& ASSOCIATED OIL AND GAS 117

MARINE CONTRACTORS AND SUPPLY 129

MC CULLOCH 60

MCCULLOCH 34

MCCULLOCH AND VENUS 144

MICHAEL T. HALBOUTY 44, 206

MID CENTURY OIL AND GAS 128

MID STATES 74

MID-CENTURY 50

MIDLAND PRODUCTION 67

MIDWEST 159,160

MOBIL $11,15,16,111,114,145,148,166,207$

MONSANTO' 108 '

MONSANTO AND PENNZOIL UNITED 183

MONSANTO CHEMICAL $194,196,197$

MOODY PROPERTIES AND SOUTHLÁND DRILLING 131

MORAN 144

MOUND 123, 125

NEIL HANSON, EDWIN COX, MONSANTO, ET. AL. 185

NEUHOFF 187

NEWMONT OIL AND TIDEWATER OIL 142

NORTH CENTRAL OIL CORPORATION 38

OHIO 158

OIL AND GAS 61

P. A. GALLERY 53

PAN AMERICAN $26,43,44,52,63,92,93,95,112,138,151,165,168$, 203

PAN AMERICAN AND WESLEY WEST 86 


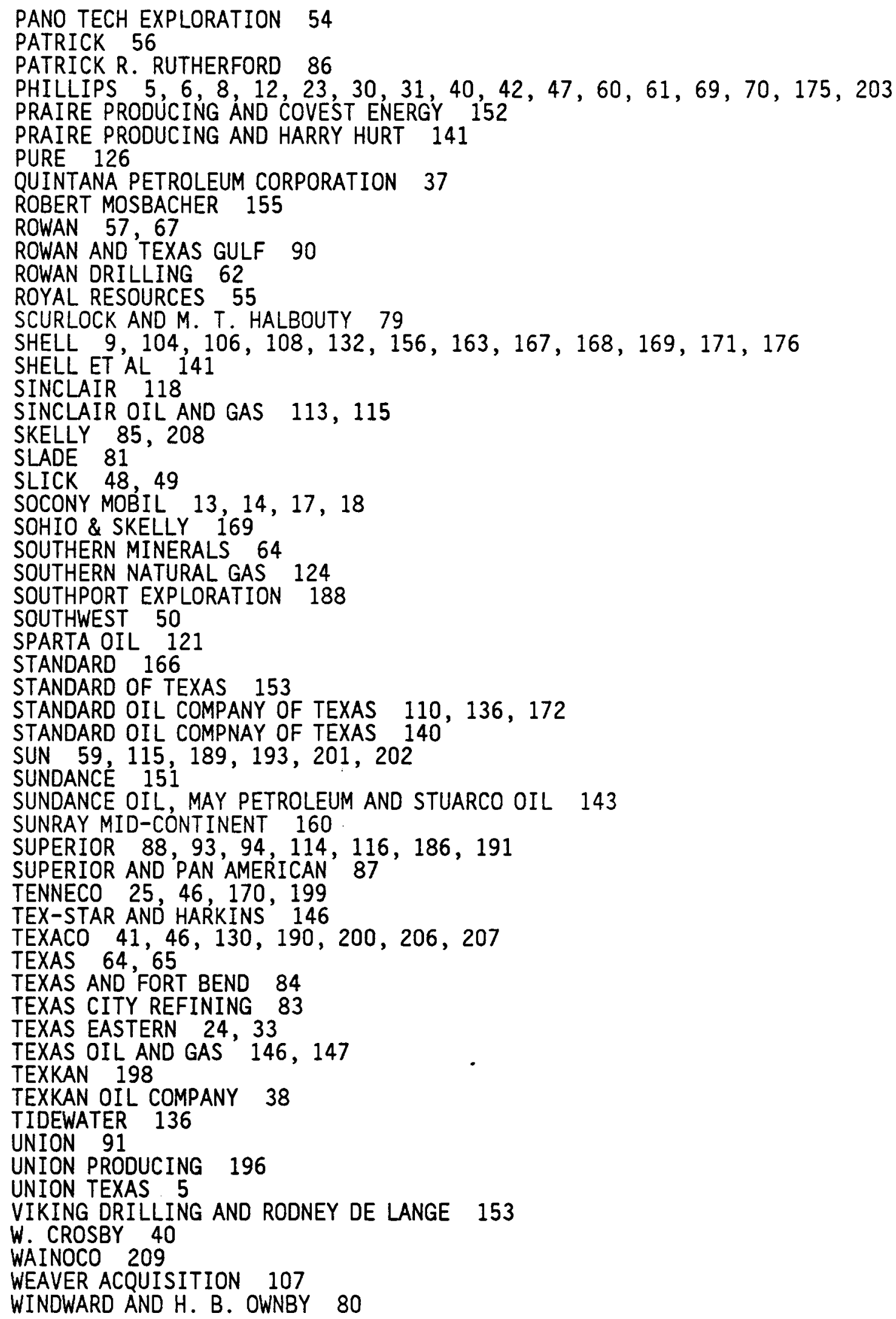




\section{S}

State

Louisiana 173, 187, 188, 189, 209

Texas $5,6,7,8,9,10,11,12,13,14,15,16,17,18,19,20,21,22$, $23,24,25,26,27,28,29,30,31,32,33,34,35,36,37,38,39,40$, $41,42,43,44,45,46,47,48,49,50,51,52,53,54,55,56,57,58$, $59,60,61,62,63,64,65,66$

\section{w}

Well names

A. B. MARSHALL 39,41

A. B. WILLIAMSON 12

A. FRIAR "A" 179

A. TACQUARD ET AL 71

A. W. ADAM 85

ALBERT JANK 103

AMERICAN FLECTCHER 25

AMERICAN LIBERTY OIL COMPANY 159

ANNABELL EVERETT 142

B. L. WILSON 88

B. LOPEZ 164

B. R. L. D. CO. 43

B. SIMON 188

B. W. TRULL ESTATE 199

BAUGH 68

BAYOU DEVELOPMENT CO. 92

BEHRENS 196

BENDER ESTATE FARM 114

BENTSEN BROTHERS C 189

BERTHA MUELLER 109

BESSIE BUDEWIG 127

BESSIE K. WELDER ET AL 157

BEST 143

BOGGAN 165

BOLIVAR TRACT "A" 76

BRENNAN BENAVIDES "B" 148

BROWN 138,180

BUCKEYE 194

BURKE-HERMAN 191

BYRNE 61

C. A. O'CONNOR 112

C. C. BELL 19

c. L. MCCASLIN 134

C. M. ARMSTRONG 99, 104, 190, 200

c. SCHORRE 180

CHARLES M. ARMSTRONG 99

CHESTERVILLE 145

CHRISTENSEN 70

CLARK ESTATE 66

CLOUDT 63

CLYDE HERRING 37 


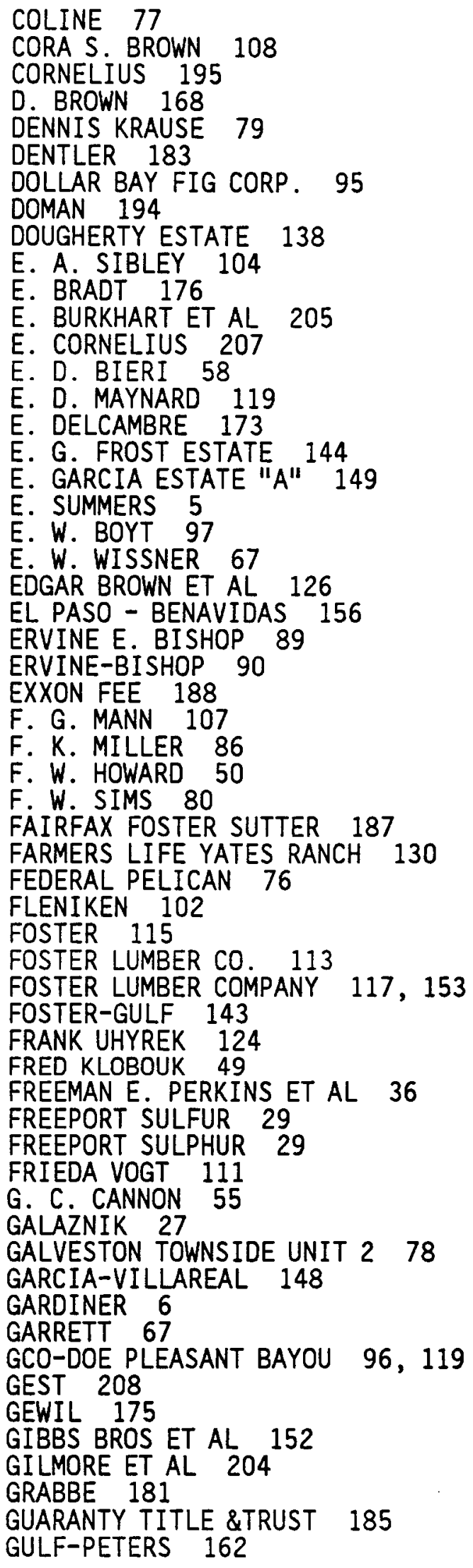




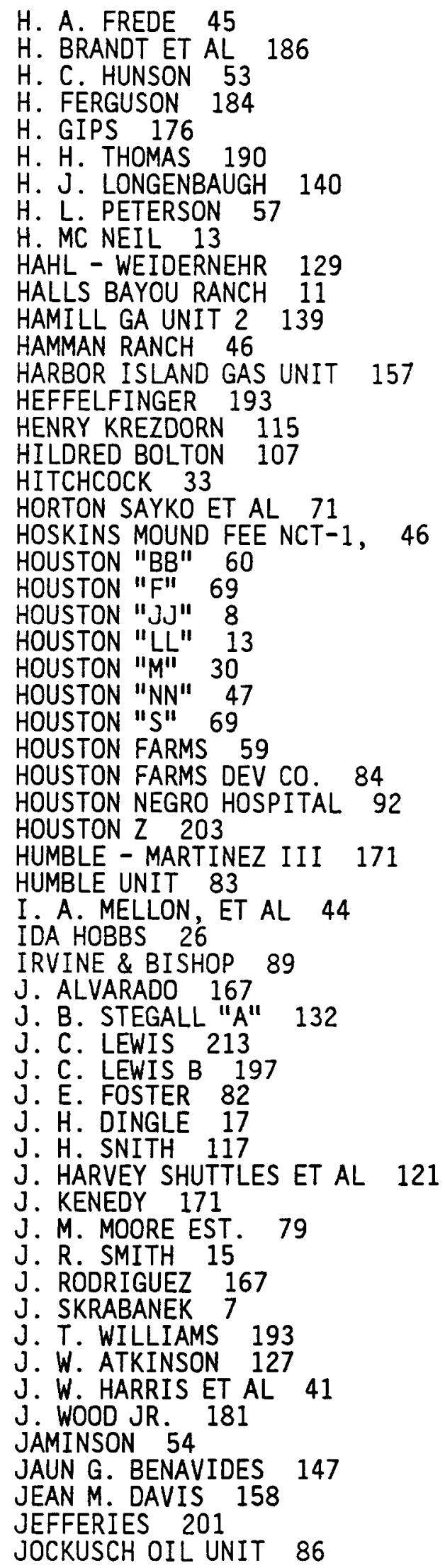




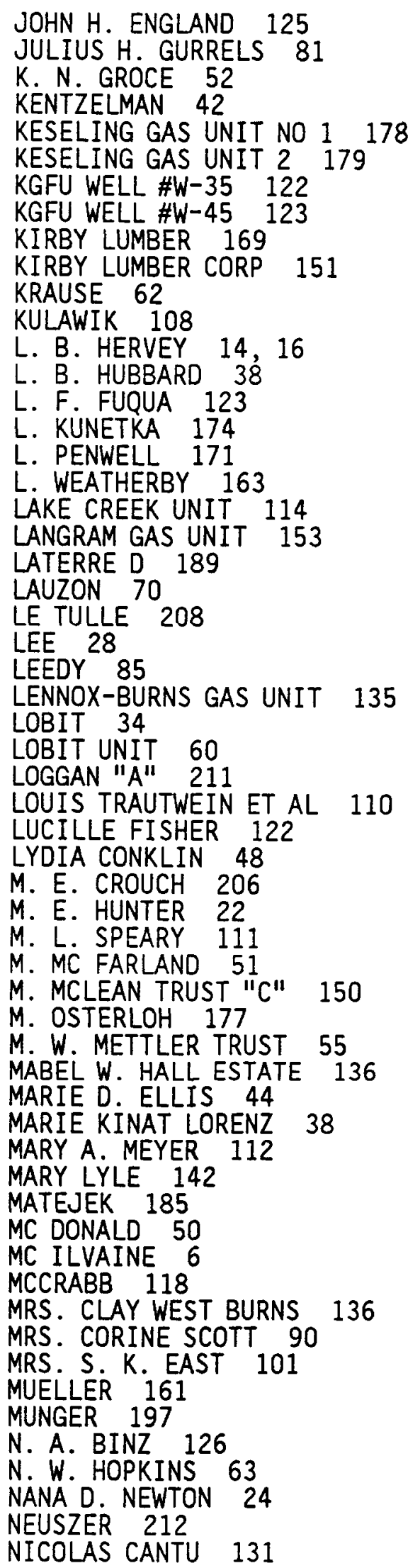


NORDHEIM GAS UNIT $15 \quad 187$

NOVAK 57

O. FRIEDRICHS 168

0 . LAUREL 162

0 . SCHROETER 184

0 . V. GUERRA "L" 147

OEHLKE 186

OSCAR BUNGE 146

P. HUEBNER 204

P. R. LIROUARD 209

P. STOCK 169

PABST "B" 31

PAUL E. WHITE "B" 131

PENNY 170

PERRYVILLE 19

PETERS ESTATE 132

PIERCE ESTATE 75, 195, 207

PIERCE ESTATES 206

POTTER 35

POTTHAST 202

POTTMAST 196

R. A. HARTMAN 109

R. B. PETERS 93

R. G. JACOBS 105

R. J. LOSTRACCO 65

R. J. ROEDER 102

R. VIEMAN 7

R. WHITE 182

RAFAELA DE MUNOZ 155

RALPH W. VIEMAN 48

RAMSEY STATE FARM 64

RANDALL CARTWRIGHT 144

RETRIEVE STATE FARM 16,18

RETRIEVE STATE FARM TRACT \#5. 42

RHODES 54163

RIO FARMS INC. 172

ROBNETT 40

ROYCE PASSMORE 134

RUGLEY 205

S. EAST 172

S. G. MARSHALL - STATE 130

S. GARCIA 154

S. H. GREEN 20

S. K. EAST 100

S. MOLLER 56

SALLIE BROWN KENNELY 81

SANGER HEIRS 137

SARA WEST HEIRS ET. AL. UNIT \#1 82

SAUERBRUNN ET AL 116

SCHENCK 175

SEALY GAS UNIT 141

SHARP CORPORATION 83

SHELL POINT 59

SHERRILL GAS UNIT 203

SOLON L. REEVES ET AL 66

SOUTH GILLOCK COMM. 95

STATE LEASE 51000 BLOCK 3261

STATE TRACT 122160 
STATE TRACT $125 \quad 156$

STATE TRACT $218 \quad 159$

STATE TRACT $81 \quad 34$

STATE TRACT $96 \quad 160$

STATE-LAGUNA MADRE 201

STEWART 88

SUPERIOR OIL COMPANY FEE 93,94

T. BRYSCH 177

T. E. FULBRIGHT 155

T. E. MOSTYN 116

T. E. SLONE 198

T. E. SPARKS 139

T. H. THEISS 9

T. L. SMITH HEIRS 52

T. M. BROOKSHIRE 135

T. MARTIN 25, 32

T. MARTIN FEE 58

T. P. HALEY TRUST 146

T. PETRUCHA 166

TACQUARD "A" 23

TEXAS STATE LEASE 5303421

TOMBALL GAS UNIT $11 \quad 118$

TRIANGLE 37

UNIVERSITY OF TEXAS 72,73

V. L. PUIG 149

VIRGINIA RAGSDALE SEC 120129

W. D. MUNSON 49

W. H. CARROLL 106

W. H. COON FEE 74

W. MCCAMBELL 170

W. MOOTHART 166

W. N. MOORE 54

W. NELSEN 191

W. OROEHL 176

W. V. ROELL 105

W. W. FONDREN ET AL 198

W. W. FONDREN, JR., ET AL 212

W. W. LANGHAM 152

WARD-BYERS 22

WEITING 200

WESTERLAGE UNIT 74

WHITE-FROST 10

WILBERT GOEBAL ET AL 141

WILLIAM HUBBEND 128

WILLIAMS 133

WILSON 40

WINTON UNIT 87

WIRT DAVIS 151

WYLIE 192

10078

11398

$118 \quad 165$

21211

$309 \quad 166$

$310 \quad 91$

34298

49

$49 \quad 210$ 
Index

$\begin{array}{ll}72 & 210 \\ 76 & 202\end{array}$

$84 \quad 210$

Page 231 